DESS Développement et Coopération

Département de Sciences Politiques

Université Paris I - La Sorbonne

Année Universitaire 1998 - 99

\title{
Le Principle Common Programming ENTRE DISCOURS ET REALITE
}

\section{L'enjeu de la coordination de l'Aide en Afghanistan}
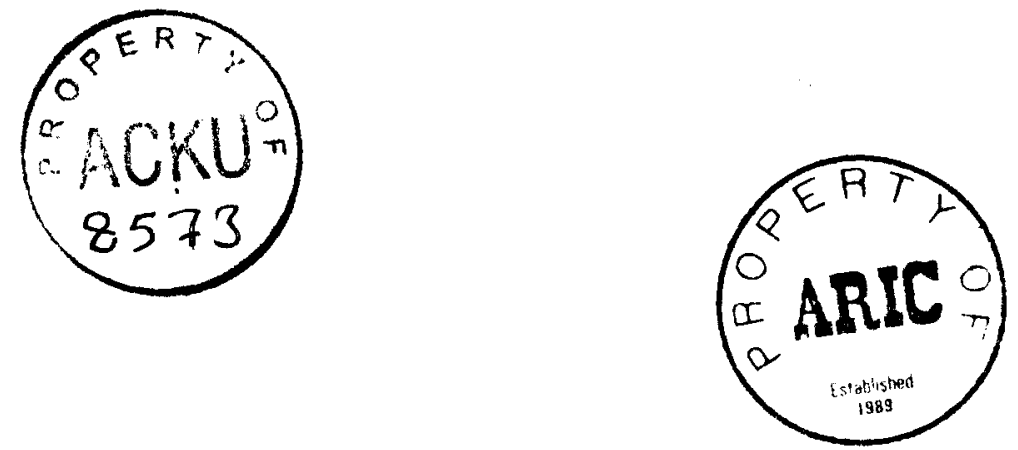

Mémoire de fin d'études

Nathalie Grimoud

Directeur de mémoire : M. Albert Bourgi 


\section{PREMIERE PARTIE : PRESENTATION DES ACTEURS}

I. Les ONG

p. 05

1.1. Typologie

1.1.1. Les ONG internationales

p. 05

1.1.2. Les ONG islamiques

1.1.3. La prolifération des ONG Afghanes

p. 06

p. 06

p. 06

\subsection{La coordination}

p. 07

II. Le CICR : un acteur à part

p. 08

III. LES NATIONS UNIES

p. 08

3.1. Les activités humanitaires et de développement

p. 08

3.1.1. Présentation des agences

3.1.2. L'évolution de la coordination au sein du système

p. 08

3.2. Les activités politiques des Nations Unies

p. 08

3.2.1. L'absence de dialogue avec le versant humanitaire

p. 10

p. 10

3.2.2. La confusion entre les mandats politiques et humanitaires des Nations Unies

p. 10

IV. LES DONATEURS

p. 11

4.1. Un ensemble hétérogène

p. 11

4.2.Des intérêts divergents

4.3. Les incohérences des bailleurs de fonds

4.4. Le dialogue entre les donateurs

V. CONCLUSION

Résumé du processus de coordination

\subsection{Les difficultés au sein de l'insitution}

1.1.1. Les crises complexes

p. 16

1.1.2. Urgence développement : la difficile transition

p. 16

1.1.3. La crise financière 
1.2.2. L'élaboration du cadre stratégique

$\begin{array}{ll}\text { 1.3. Les tentatives de coordination depuis Islamabad } & \text { p. } 23\end{array}$

1.3.1. Le poids de l'UNOCHA p. 23

$\begin{array}{ll}\text { 1.3.2. La réticence des agences } & \text { p. } 24\end{array}$

$\begin{array}{ll}\text { II. LA PRESSION DES DONATEURS } & \text { p. } 25\end{array}$

$\begin{array}{ll}\text { 2.1. Leur place dans le processus } & \text { p. } 25\end{array}$

$\begin{array}{ll}\text { 2.2. Leurs objectifs } & \text { p. } 26\end{array}$

$\begin{array}{ll}\text { 2.3.Humanitaire et politique } & \text { p. } 28\end{array}$

2.3.1. Une aide neutre? $\quad$ p. 29

2.3.2. Vers une politisation accrue de l'aide $\quad$ p. 30

2.3.3. La sécurité comme prétexte $\quad$ p. 31

2.3.4. Des principes « en principe» à la conditionnalité $\quad$ p. 32

2.3.5. Le PCP : un facteur de politisation? $\quad$ p. 33

$\begin{array}{ll}\text { I. LES MODALITES DE LA CONSULTATION } & \text { p. } 34\end{array}$

II. LES STRUCTURES MISES EN PLACE $\quad$ p. 35

$\begin{array}{ll}\text { 2.1. Au niveau national } & \text { p. } 35\end{array}$

2.1.1. A.P.B. p. 35

2.1.2. A.S.G. p. 36

2.1.3. Les groupes thématiques $\quad$ p. 36

$\begin{array}{ll}\text { 2.2. Au niveau régional } & \text { p. } 36\end{array}$

$\begin{array}{ll}2.2 .1 \text {. Kaboul } & \text { p. } 37\end{array}$

$\begin{array}{ll}\text { 2.2.2. La région nord } & \text { p. } 37\end{array}$

2.2.3. Le Hazarajat: coordination par le haut $\quad$ p. 38

2.3. L'Appel Consolidé $\quad$ p. 41

2.3.1. Définition p. 41

2.3.2. L'Appel et le PCP $\quad$ p. 41

$\begin{array}{ll}\text { 2.3.3. Les résultats de l'Appel } & \text { p. } 42\end{array}$

2.3.4. La définition de stratégie commune : un véritable faux semblant $\quad$ p. 42

$\begin{array}{ll}\text { 2.4. Un bilan mitigé } & \text { p. } 45\end{array}$

$\begin{array}{ll}\text { CONCLUSION } & \text { p. } 47\end{array}$

$\begin{array}{lr}\text { BIBLIOGRAPHIE } & \text { p. } 49\end{array}$

TABLE DES ANNEXES p. 51 


\section{INTRODUCTION}

Cette étude a été réalisée lors d'un stage de cinq mois au sein de MADERA, Mission d'Aide au Développement des Economies Rurales en Afghanistan, la majeure partie de mon travail s'est déroulée dans le bureau de Peshawar, j'ai cependant été amenée à me déplacer en Afghanistan, dans la région Est et le Hazarajat. Le cadre de cette recherche est particulier puisqu'elle n'est pas le fruit d'un travail effectué à proprement parlé au sein de l'association.

MADERA est une ONG créée en 1988 dont l'objectif principal était de favoriser le retour des réfugiés dans leur village d'origine par des actions agro-pastorales et génie civil mais qui réoriente actuellement son action vers des problématiques de développement à long terme et ne travaille plus seulement auprès des anciens réfugiés. Elle occupe en raison de son ancienneté et de l'ampleur de ses programmes une place importante sur l'échiquier de l'assistance à l'Afghanistan. Attentive à l'environnement dans lequel elle travaille, elle a été fortement, impliquée, dans le processus que nous allons étudier, notamment à travers la personne de son ancien directeur.

Il s'agit d'une tentative d'amélioration de l'efficacité de l'assistance internationale destinée à l'Afghanistan à travers la mise en place d'un processus de coordination, connu sous le vocable anglais de Principled Common Programming (PCP), expression rarement traduite par son équivalent français: Programmation commune basée sur des principes. Nous insistons sur le terme de processus qui reflète bien le caractère évolutif de cette initiative, qui débuta en 1997. La première difficulté a donc consisté à comprendre la nature et l'objet de ce processus. Le sens donné au terme de coordination est large, il s'agit de l'organisation des activités des différents groupes en vue d'une meilleure efficacité de chacun et d'une plus grande cohérence de l'ensemble.

Le processus de programmation commune tente d'établir par une architecture élaborée des mécanismes de concertation entre les acteurs de l'aide. Il est né de l'impulsion du siège des Nations Unies qui cherche à définir son rôle dans les nouvelles crises auxquelles l'institution est confrontée et s'est concrétisé suite à la pression des bailleurs de fonds bilatéraux impliqués en Afghanistan.

Nous sommes partis d'un double constat. D'une part peu de personnes avait une connaissance précise de ce processus complexe et les réactions à sa seule évocation sont des réactions de rejet quasi épidermique de la part de nombre d'acteurs de l'aide. D'autre part en dépit de la somme incommensurable de débats et de réunions consacrés à ce sujet, il est très difficile d'en percevoir les effets concrets et aucune évaluation globale n'en a été réalisée à ce jour. Il nous a donc semblé nécessaire de dépasser une approche superficielle qui se limite au constat de l'absence de résultats sur le terrain, pour tenter de comprendre quels sont les véritables enjeux d'une telle coordination.

L'objet de ce travail est donc de déterminer quels sont les enjeux de ce processus pour les acteurs en présence : les bailleurs de fonds, les Nations Unies et les ONG et de voir en quoi ces enjeux influent sur sa mise en œuvre. Il s'agit de comprendre pourquoi les effets sur le terrain 
sont si peu perceptibles et pourquoi l'enjeu humanitaire : l'aide à la population passe au second plan.

La première partie de ce travail consiste donc en une présentation des différents acteurs et des coordinations existantes au début du processus qui permettra de situer la scène complexe de la communauté de l'aide à l'Afghanistan. Nous nous attacherons ensuite à mettre en relief à travers l'histoire de ce processus, les motivations et les enjeux qui le sous-tendent. Pour ce faire, nous porterons plus particulièrement notre attention sur les deux catégories d'acteurs qui en sont à l'origine : les Nations Unies et les bailleurs de fonds. Nous verrons ainsi que c'est davantage la reprise en main de l'assistance internationale que la coordination qui est en jeu. La troisième partie permettra, à travers l'étude des mécanismes et des structures mis en place, de montrer les effets pervers du processus.

\footnotetext{
Avertissement :

Ce travail a reposé sur l'étude de la manne de documentation concernant le sujet mais aussi sur une série d'entretiens non directifs avec des personnes représentatives des différentes catégories d'acteurs. La plupart de mes interlocuteurs m'ayant demandé de conserver la confidentialité de leur propos, je n'ai pas reproduit les entretiens, ceci explique aussi le fait que nombre de citations n'aient aucune référence.
} 


\section{PREMIERE PARTIE : PRESENTATION DES ACTEURS DE L'AIDE EN AFGHANISTAN}

Cette première partie a pour but de présenter les différentes catégories d'acteurs amenées à prendre part au processus de coordination, afin de situer chacun dans le paysage de l'aide en Afghanistan. La première caractéristique en est la complexité, si nous retrouvons les protagonistes classiques de l'assistance, les ONG, le CICR, les Nations Unies, les bailleurs de fonds, il convient de souligner que leur rôle et leurs objectifs relatifs ont évolué, les relations établies à l'intérieur des groupes et entre eux sont le fruit d'une histoire longue et houleuse dont l'étude dépasse le cadre de ce travail mais qu'il convient de garder à l'esprit. Nous porterons une attention plus particulière aux mécanismes de coordination établis au sein de chacun.

\section{Les ONG}

\subsection{Typologie}

Elles sont présentes en Afghanistan depuis le début de l'intervention soviétique, nous ne retracerons pas ici l'histoire de leur évolution depuis l'entrée clandestine sur le territoire de quelques médecins apportant leur soutien à la résistance jusqu'à la multiplication du nombre d'agences de plus en plus professionnelles.

Il est très difficile d'obtenir des sources statistiques fiables ${ }^{1}$ sur le système d'assistance, les chiffres que nous indiquerons sont donc sujet à caution et controverse, même si nous avons tenté de les confirmer par recoupement. Aujourd'hui on recense entre 252 et $185^{2}$ ONG dont 61 ONG internationales et 124 à 204 Afghanes.

Les $\mathrm{ONG}$ sont la pierre angulaire de l'aide extérieure à l'Afghanistan, elles mettent en œuvre selon les années environ $75 \%$ des fonds alloués à l'Afghanistan. Ainsi en 98 les budgets cumulés des seules ONG membres d'une coordination représentaient 125.5 millions US\$ sur un total de 203 millions US\$. Les ONG internationales disposent de 64,4\% des fonds, les Afghanes $28 \%$, les islamiques $7,6 \%$ si l'on prend en compte les seules ONG membres d'une coordination sachant que $22,5 \%$ du total sont gérés par des ONG n'étant membres d'aucune coordination.

Leurs principales sources de financements sont la Communauté européenne $(28 \%$ des financements ONG en 98), les financements bilatéraux ( $20 \%$ dont une large partie de pays membres de l'Union Européenne), enfin $22 \%$ des fonds qui leur sont alloués transitent par les agences des Nations Unies.

La typologie la plus usitée consiste à distinguer les ONG afghanes islamiques et internationales.

\footnotetext{
${ }^{1}$ Cf. Annexe ${ }^{\circ} 3$ : financements de l'aide à l'Afghanistan

2 Source " Database of the Ngo activity, 1999 », ACBAR, Peshawar, 1999
} 


\subsubsection{Les ONG internationales}

Nous utiliserons ici une définition, par la négative d'une ONG internationale qui désigne toute ONG qui n'est pas afghane. Les ONG afghanes sont définies comme ayant seulement un bureau en Afghanistan. Les critères habituels de désignation d'une ONG internationale retenus par les Nations Unies, à savoir travaillant dans au moins trois pays, ne sont donc pas valables ici, en effet certaines ONG ont été créées spécifiquement pour travailler en Afghanistan et ne se sont pas engagées sur $\mathrm{d}^{\text {'autres terrains }}{ }^{3}$.

\subsubsection{Les $11 \mathrm{ONG}$ islamiques}

Elles ont leur bureau principal dans un pays musulman ou en drainent des fonds, elles se distinguent des autres ONG internationales. De taille moyenne à importante leurs budgets cumulés atteignent 7,6\% du total des ONG. Elles sont regroupées au sein de l'Islamic Coordination Council (ICC) depuis 1985. Ayant pour objectif de fournir un forum de discussion à ses membres, cet organe sera impliqué dans le processus. Il dispose de représentants au sen de l'organe national de coordination et soulève le problème de la non représentation des bailleurs de fonds issus des pays musulmans.

\subsubsection{La prolifération des $O N G$ afghanes}

\section{- Historique}

Il n'existait avant 88 qu'une dizaine d'ONG afghanes, c'est sous l'impulsion des Nations Unies et notamment de l'UNOCA, qu'elles se sont multipliées, il s'agissait de créer des entités afghanes d'une part afin de court-circuiter les ONG internationales et d'autre part en vue de renforcer les capacités des Afghans. L'Afghanisation était fondée sur des arguments mettant en relief la meilleure adaptabilité au contexte socio-culturel, la plus grande implication des Afghans et le moindre coût des actions entreprises. Cependant, ce processus s'est heurté à des obstacles qui n'ont pas été pris en compte à savoir le cruel déficit en cadres Afghans et leur faible qualification, l'absence de sensibilisation au concept même d'ONG. Si bien que cette tentative s'est soldée par la création d'une multitude d'entités, souvent par des personnes ayant des relations avec du personnel local onusien et des ONG. Les corruptions et détournements ont été nombreux - attention ceci n'est pas l'apanage des ONG afghanes mais le risque y est accentué par le manque de suivi et de contrôle. Comme le fait remarquer un de nos interlocuteurs « at that time there were people settled in Peshawar just signing cheques » - et il était très facile d'obtenir des fonds et l'on ne compte plus le nombre d'écoles qui ont été financées plusieurs fois mais qui n'existent que sur le papier.

\section{- Le résultat aujourd'hui de l'Afghanisation}

La restriction des fonds amène à une compétition exacerbée entre les organisations. Nombre d'ONG afghanes ont eu une durée de vie limitée, un petit nombre d'entre elles a démontré de véritables capacités de travail, certaines $\mathrm{ONG}$ internationales ont réussi avec succès leurs transitions et sont maintenant totalement gérées par les Afghans. Cependant, le jugement des expatriés sur les ONG afghanes est souvent sévère, hormis quelques personnes présentes depuis longtemps, beaucoup sont incapables de distinguer les quelques ONG de qualité et

${ }^{3}$ Exemple Swedish Committee for Afghanistan, MADERA, DACAAR, Solidarité Afghanistan Belgique, Norvegian Committee for Afghanistan 
répondant aux critères communs de définition d'une ONG de la masse des contractants des Nations Unies, travaillant au coup par coup pour des projets de génie civil financés par les agences onusiennes .

\section{$\underline{1.2 \text { La coordination }}$}

Les ONG ont depuis longtemps pris en charge leur propre coordination. Des organes à cet effet, existaient déjà ainsi qu'un début de concertation entre les différents acteurs bien avant que la coordination ne devienne le thème en vogue sur la scène de l'assistance pour l'Afghanistan.

On dénombre 5 agences de coordination d'ONG ${ }^{4}$ regroupant $2 / 3$ des associations travaillant en Afghanistan selon des critères de nationalités, religieux ou d'aires géographiques.

- ACBAR est l'organisation la plus active et possédant le plus grand budget, créée en 1988, basée à Peshawar et regroupe, en 1999, 75 organisations sans critères de discriminations, mais elle a établi une liste de conditions à l'acquisition de la qualité de membres.

- ANCB est la coordination qui regroupe le plus d'agences, elle rassemble 106 membres, créée en 91 elle est spécifique aux ONG Afghanes.

Deux organes particuliers à des régions ont été créés, ils se justifient par l'éloignement des régions concernées.

- NCB est une coordination régionale composée de 12 membres elle est basée à Hérat et existe depuis 1995.

- SWABAC a été créée en 88 , elle rassemble 81 agences travaillant dans le sud, l'ouest Afghan et le Baloutchistan, ce sont principalement des ONG afghanes, dont certaines basées à Kandahar, présentent la particularité, d'être liées au pouvoir Taliban.

Les relations entre les agences de coordination sont marquées «par un manque de confiance et de coopération $\nu^{5}$ dus à des conflits de personnes et professionnels, ainsi il existe des divergences notables concernant les relations avec les Taliban. Afin de remédier à ces divergences, un audit des coordinations a été conduit en 99 sous la pression des donateurs. Il préconise l'établissement d'une coordination unique, ces conclusions font évidemment l'objet de désaccord entre les agences. Cependant une force de travail constituée de membres des conseils d'administration des différents organes se réunit mensuellement afin de rechercher des modalités de transition vers un organisme commun.

Outre ces organes, il faut souligner l'importance de la collaboration informelle sur le terrain et l'existence de différents organes établis pour s'assurer de la cohérence des actions engagées que ce soit sur des thèmes précis, techniques, ou en vue de l'harmonisation des approches au sein d'une aire. Ainsi dans la région Est, les ONG financées par la DG1 ont déterminé 7 zones cibles de rapatriement dans lesquelles une division du travail a permis de mieux répondre aux besoins.

\footnotetext{
${ }^{4} \mathrm{Cf}$. annexe $\mathrm{n}^{\circ} 5$ 《 role and activities of coordinating bodies 》

${ }^{5}$ A Strand, A.Najimi, N. Lander « NGO cooperation open for debate, interim report and preliminary recommandations, commisssioned by ACBAR, ICC ANCB, Peshawar 1999
} 


\section{Le CICR : un acteur à part}

Il comptabilise en volume financier environ $20 \%$ de l'aide dispensée en Afghanistan, présent en permanence depuis 1986, il intervient principalement dans les domaines de l'urgence et de la santé. Il jouit d'une reconnaissance de la spécificité de son mandat par l'ensemble des acteurs. Il s'est, depuis le début du processus, tenu à l'écart, se parant de son mandat spécial, il assiste en tant qu'observateur à certaines réunions mais revendique une indépendance totale ${ }^{6}$ qui va à l'encontre de la volonté affirmée d'établir une stratégie commune. Il est important de souligner que sa position particulière est acceptée et reconnue par tous. En outre, il participe à des coordinations opérationnelles sur des thèmes précis telle que l'assistance aux déplacés. Il est aussi remarquable que si la neutralité reconnue du CICR le tient à l'écart du PCP, le fait que les ONG soient signataires du code de conduite qu'il édite ne leur offre pas la même liberté d'action.

\section{LES NATIONS UNIES}

\subsection{Les activités humanitaires et de développement}

\subsubsection{Présentation des agences}

Les agences présentes en Afghanistan sont la FAO, section agriculture et élevage, Habitat, UNDP dont CDAP (Compréhensive Disabled Afghan Program), UNDCP( UN drug control program), UNHCR( Haut commissariat pour les réfugiés), UNICEF, le programme alimentaire mondial, (PAM) et l'organisation mondiale pour la santé (OMS), UNOPS (Office of projects services), UNESCO, Office International des Migrations (IOM) . La majorité des fonds des agences est versée à des ONG qui mettent en œuvre les programmes. L'analyse des financements est rendue quasi-impossible par l'opacité des agences qui refusent de diffuser les renseignements. Il est cependant notoire que les frais administratifs de ces agences oscillent entre 60 et $70 \%$ de leur budget. Le PAM a le plus gros budget et travaille essentiellement sur fonds propres.

\subsubsection{L'évolution de la coordination au sein du système}

\section{- 1988-97 : duplication de coordination}

L'UNDP avait jusqu'en 1997 la charge de la coordination des agences de développement, présent en Afghanistan depuis les années 70 son rôle consiste à financer des projets sans les mettre en œuvre. Il n'a pas de stratégie propre et répond au coup par coup aux demandes de financements des ONG et agences, ainsi il finance la majeure partie de UNCHS, il est aussi contributeur de la FAO, il était censé jouer un rôle de coordinateur des activités de réhabilitation et de développement mais n'a jamais réellement rempli cette fonction. Doté à partir de 91 d'un représentant résident, il a eu une place active dans la promotion des programmes de réhabilitation. La création de UNOCA a entraîné naturellement une compétition entre les deux agences.

L'UNOCHA a succédé en 1992 à l'UNOCA (bureau pour la coordination des programmes humanitaires et d'assistance économique des Nations Unies pour l'Afghanistan) créé en 1988.

\footnotetext{
${ }^{6}$ Le représentant du CICR à Ashgabat a affirmé le refus d'être coordonné par les Nations Unies
} 
Celui-ci avait à l'origine pour objectif de coordonner les fonds des UN destinés à 1 'action humanitaire et au développement, il était doté d'importants fonds : l'AETF (Afghan Emergency Trust Fund) mais rapidement il s'est détourné de sa fonction première en se contentant de répondre aux sollicitations pour redistribuer ses fonds et en abandonnant son travail de coordination et planification. De plus, sa création a été mal perçue par des agences telles que UNDP ou HCR qui se voyaient mieux placées qu'une nouvelle entité pour prendre la tête des activités des Nations Unies. En 1992, il est placé sous l'égide du département pour les Affaires Humanitaires nouvellement créé, son mandat est restreint à la coordination des activités humanitaires. Cependant, les différentes agences se sont entre temps renforcées ayant notamment accès à des fonds directs des donateurs et ne sont donc plus dépendantes de UNOCHA, elles continuent «à se coordonner avec lui mais sont beaucoup moins prêtes à être coordonner par lui»s. ${ }^{7}$ L'UNOCHA est, en 96, beaucoup plus perçu comme une agence avec ses propres programmes (déminage) et procurant des services (aviation, radio) que comme un organisme de coordination.

L'Afghanistan est donc doté d'un double mécanisme de coordination des Nations Unies, renforçant la séparation artificielle entre activités humanitaires et développement. Cette situation contribue à l'entretien d'un climat où toute collaboration semble impossible.

\section{- Des relations de compétition}

Chaque agence réalise ses programmes, avec sa propre stratégie, (lorsqu'elle existe). Il n'est guère possible d'avoir une vision d'ensemble des activités d'aide des Nations Unies, ni de l'objectif de l'institution dans le pays. Il existe certes un document qui rassemble les différentes demandes de fonds des agences : l'Appel Consolidé, sa préparation est organisée par UNOCHA mais celui-ci n'est qu'une liste de projets.

Les relations entre agences relèvent de la compétition exacerbée, aucun dialogue, aucune réflexion commune ne sont envisagés. Toute tentative de discussion est perçue comme une ingérence, lorsqu'une agence recueille de bons résultats elle est en but aux jalousies professionnelles. Le manque de coordination a des conséquences négatives sur les programmes, lorsque deux agences travaillent dans les mêmes zones. Ainsi le HCR tend à fournir gratuitement des biens, lorsqu'il le fait dans une zone où d'autres organisations travaillent en tentant de renforcer la participation des communautés, ces distributions peuvent remettre en cause des projets. S'ils en sont conscients, les fonctionnaires onusiens ne remettent jamais en cause leur travail et se réfugient derrière la variété des mandats pour excuser ces lacunes et l'absence de concertation.

Le caractère désordonné des réponses onusiennes aux politiques des Taliban a été dénoncé en 1997 lorsque l'OMS a accepté de réhabiliter l'hôpital de Rabia Balki destiné aux femmes à Kaboul alors que les autres acteurs luttaient pour qu'elles conservent l'accès aux hôpitaux mixtes. Tout d'abord, en raison de son incapacité à coordonner ses propres agences entre elles. Ainsi le rapport du sous secrétaire général $\mathrm{M}$. de Mello publié en mars 98 suite à une mission sur le terrain en février relève de nombreuses incohérences. Il souligne la variété de réponses et d'attitudes face aux Taliban allant d'une position stricte de principes à une approche par trop pragmatique. «Ainsi, le gouverneur de Kandahar se vante d'avoir réussi a faire financer un projet par une agence alors qu'une autre l'avait refusé pour une question de principe» ${ }^{8}$. Il constate que

${ }^{7}$ A. Donini The policies of mercy: UN coordination in Afghanistan, Mozambique and Rwanda, 1996

${ }^{8}$ S.V. De Mello " Mission report in Afghanistan », New york, Mars 98 
les sièges des agences ont accueilli l'initiative de Common Programming avec frilosité. Il souligne combien il sera difficile de «réconcilier les priorités des différentes agences chacune ayant ses propres politiques et intérêts ».

Les relations avec les ONG n'étaient en général pas bonne sur le terrain, elles ne jouissent à leurs yeux d'aucune capacité à intervenir auprès des populations, les ONG reprochent aux Nations Unies «leur manque de professionnalisme et d'avoir une attitude de condescendance et d'arrogance à leur égard».

\subsection{Les activités politiques des Nations Unies}

La nature de l'action des Nations Unies en Afghanistan est d'autant plus opaque qu'aux volets humanitaires et de développement s'ajoute un volet politique. Celui-ci est pris en charge par le OSGAP (Office for the secretary general for Afghanistan and Pakistan) puis par UNSMA à partir de?. Mais l'échec du volet politique des activités des Nations Unies, tant à ramener le dialogue entre les différentes factions, qu'à limiter les interférences des puissances extérieures, a aussi des conséquences sur la délivrance de l'aide.

\subsubsection{L'absence de dialogue avec le versant humanitaire}

Il était fondamental pour les acteurs de l'aide de mettre en avant le fait que leurs activités n'avaient rien de politique et n'étaient en rien liées avec ceux qui menaient les négociations à ce niveau là. Ainsi, la coordination entre le versant politique et humanitaire des activités des Nations Unies était jusqu'ici très limitée. Les relations étant des relations de méfiance réciproque, le politique reprochant aux humanitaires de ne rien comprendre à la politique et les seconds préférant se tenir à distance des premiers afin de ne pas compromettre leur image de neutralité et de ne pas perdre leur crédibilité. Cependant, de 1991 à 1994, UNOCHA et OSGAP ont été dirigés par la même personne, ce qui a permis un meilleur dialogue entre les différents acteurs et de pallier en partie au fait que les personnes chargées des négociations politiques oublient souvent de consulter les acteurs humanitaires dont la connaissance du terrain pourrait être utile. Ainsi, il a été reproché au représentant spécial de ne pas aborder le «problème des conditions de travail des acteurs humanitaires, ni les questions relatives aux droits de l'homme et à l'égalité des sexes» ${ }^{9}$. Les politiques empêtrés dans de grandes négociations ne se préoccupent pas des problèmes rencontrés sur le terrain, ainsi lors de la publication de l'édit du Mahram interdisant à toutes les musulmanes de se déplacer sans un membre de leur famille sur le territoire Afghan, l'ensemble de la communauté de l'aide a demandé une intervention de l'UNSMA

\subsubsection{La confusion entre les mandats politiques et humanitaires des Nations Unies}

La question des liens entre activités politiques et humanitaires est une question d'autant plus complexe dans le contexte afghan que ces deux versants ont été longtemps liés. La confusion aboutit à un grave écueil: celui de faire porter à la communauté de l'aide internationale une responsabilité qui ne lui revient pas : apporter la paix. A cet égard les débats lors de la conférence d'Ashgabat sont révélateurs. L'élaboration du futur cadre stratégique, se fonde en partie sur cette erreur conceptuelle, elle dresse le constat de l'échec de la communauté

\footnotetext{
${ }^{9}$ M. Keating « Aid politics : dilemnas of humanitarian assistance $»$ in Essential field guide to humanitarian and conflict zone : Afghanistan, ICRC, Geneva 1998
} 
internationale dans son ensemble sans faire de distinction entre les buts politiques de la OSGAP et le but humanitaire des ONG et agences spécialisées des Nations Unies. Un autre risque est celui d'une politisation à outrance des activités humanitaires et de développement et une possible perversion de l'aide par la conditionnalité.

\section{LES DONATEURS}

\subsection{Un ensemble hétérogène}

Comme les autres groupes, il présente une grande variété tant en ce qui concerne les volumes de financement ${ }^{10}$, les choix des programmes que les motivations à intervenir. Le principal bailleur de fonds est aujourd'hui la Communauté Européenne, viennent ensuite les Etats Unis. Des pays européens financent une part importante de l'aide par le canal bilatéral : Le Royaume Uni, La Suède, les Pays Bas, viennent enfin la Norvège et le Canada. Les pays musulmans apportent leur aide par le canal d'ONG islamiques.

L'évolution des financements destinés à l'Afghanistan a suivi celle de l'intérêt des puissances extérieures pour le conflit. Ainsi les années 1985 à 1989 ont été des années fastes avec une aide annuelle dépassant les 200 millions US\$, le premier tournant consiste en la réduction de la manne des financements américains après 1989. L'Union Européenne prend le relais avec notamment la création d'ECHO (European Commission Humanitarian Office) en 1992. Si le discours ambiant consiste à mettre en exergue une tendance à la baisse des fonds pour l'Afghanistan, l'analyse des statistiques produite par ACBAR tend à montrer le contraire ; à l'exception des années 94 et 96 où le volume de l'aide transitant par les ONG est resté environ constant. En revanche, les agences des Nations Unies, victimes d'une très mauvaise image, ont davantage souffert de la baisse des financements. Les grandes ONG internationales travaillant exclusivement en Afghanistan ont pu maintenir leur niveau d'activité grâce aux fonds européens ou bilatéraux.

\subsection{Des intérêts divergents}

Les bailleurs de fonds ont des motivations à intervenir basées sur l'histoire des relations avec le pays. Les Etats-Unis et le Royaume Uni ont clairement montré lors des dernières années le caractère politique de leur assistance ${ }^{11}$ en imposant des conditions à leur financement.

Les autres « financeurs » prétendent agir en toute neutralité. Il ne faut pas ici tomber dans le piège des ONG qui ont tendance à s'arroger le monopole de la philanthropie et à penser qu'elles sont les seules à intervenir sur une base altruiste. Mais il convient de reconnaître que les représentants des donateurs ont des comptes à rendre à leurs opinions publiques. Ils doivent tenir compte des enjeux politiques. De plus, ce ne sont pas eux qui ont le monopole de la décision. Ils insistent sur la nécessité de justifier leurs budgets auprès de leur siège et au-delà des électeurs. Ainsi le représentant américain explique par la pression des organismes féministes américains, la volonté de mettre en œuvre des projets destinés aux femmes. Il met aussi en avant le problème de la production d'opium et du terrorisme. L'Europe, semble elle, davantage préoccupée par la peur d'un afflux de réfugiés ; une grande part des fonds vient de la ligne Réfugiés de la DGl

\footnotetext{
${ }^{10}$ CF Annexe dons contributions CAP99 p 16

"nous étudierons ce phénomène dans la seconde partie
} 
(direction générale). L'ensemble des donateurs insiste sur les questions des droits de l'homme, sur la distribution d'une aide ne contredisant pas les principes éthiques.

La combinaison de la médiatisation du système Taliban, et, à un niveau plus global de la restriction des fonds destinés à l'aide au développement, entraînent au milieu des années 90 , une remise en question des fonds destinés à l'Afghanistan. Les donateurs s'interrogent sur l'efficacité de l'aide dispensée, ils s'insurgent contre les duplications de projets et ont une image assez négative du travail effectué. Ils constitueront donc un puissant facteur de réorganisation.

\subsection{Les incohérences des bailleurs de fonds}

Pourtant, l'organisation même des bailleurs de fonds, leurs systèmes de distribution de l'aide, les administrations différentes sont autant d'obstacles à la coordination, les cycles de financements varient. Les coordinations ne sont pas nécessairement imposées pour améliorer la cohérence de l'aide mais pour faciliter le travail des services en charge de son administration. Ainsi, les ONG financées par la DG1 se sont vues imposées de travailler en consortium regroupant sous l'égide d'une ONG chef de file, 3 ou 4 ONG. Or, le choix des partenaires n'a pas été laissé à la discrétion des ONG mais s'est fait en fonction des dates de fin de l'actuel contrat DGl. Ainsi, des associations ne travaillant ni dans les mêmes secteurs ni dans les mêmes aires géographiques se voient regroupées. Ce qui ne facilite pas, loin s'en faut, la coordination sur le terrain. L'exemple du consortium de MADERA ${ }^{12}$ est à cet égard révélateur, il n'inclue aucun de leur partenaire habituel de travail et leur impose de travailler avec des organismes trop éloignés pour permettre une collaboration opérationnelle. Cependant, une amélioration de ce système n'est pas à exclure ainsi les ONG scandinaves sont parvenues à établir un consortium plus opérationnel.

\subsection{Le dialogue entre les donateurs}

Les bailleurs de fonds dressent eux aussi le constat du manque de coordination entre les différents acteurs. Fcepndant les prémisses d'une meilleure coordination sont perceptibles dès le milieu des années 90. Il existait depuis 1996, un regroupement des pays ayant un intérêt ou une influence en Afghanistan. Ses réunions se tiennent à New York et à la demande du secrétaire général des Nations Unies; elles ont un agenda politique , y sont présents des pays n'intervenant pas comme bailleurs de fonds dans le pays. Des mécanismes informels de coordination sur le terrain étaient en place ainsi ils participent à la Force de Travail créée en 96. Les mécanismes de concertation entre bailleurs de fonds ont été institutionnalisés en 1997 à l'occasion de la mise en œuvre du PCP.

\section{CONCLUSION}

Le champ de l'aide internationale à l'Afghanistan est complexe, les rôles des uns et des autres ne sont pas toujours bien définis, ceci a certes permis des abus et parfois un gaspillage de l'aide. Il convient cependant de ne pas tomber dans le piège d'une critique tous azimuts du fonctionnement de l'assistance. Si la recommandation de ne pas "réinventer la roue » revient comme un leitmotiv dans les discours des promoteurs d'une meilleure coordination, il semble qu'elle n'ait pas toujours été appliquée. Une plus grande attention portée aux mécanismes de concertation en place sur le terrain aurait sans doute permis d'éviter nombre d'erreurs. Il est vrai

${ }^{12}$ les autres membres sont IRC, Handicap International et Solidarité belgique 
qu'une telle approche aurait demandé une connaissance du terrain que les personnes chargées de dessiner les mécanismes n'avaient sans doute pas. Les déficiences de la communauté internationale en Afghanistan ne relèvent pas nécessairement d'une mauvaise coordination. Il convient de distinguer d'une part les problèmes internes aux organismes, et d'autre part leurs relations. Enfin, il faut prendre en compte les facteurs extérieurs, l'Afghanistan reste un pays en guerre et on ne peut attendre de la communauté de l'aide qu'elle apporte la paix.

Le fait que la demande d'une meilleure coordination ait pour corollaire la crainte d'une réduction des financements place les acteurs en concurrence sur le marché de l'aide et ne favorise pas, loin s'en faut, un meilleur dialogue. L'analyse de la situation qui a précédé le PCP est en partie faussée ; elle occulte les réussites en ne soulignant que le déficit de coordination qui n'est qu'un volet des problèmes. Cependant, personne ne remet en cause la nécessité d'apporter davantage de cohérence au système d'assistance.

La coordination est le rêve de tous, mais les motivations de chacun sont contradictoires. La prétention à une meilleure efficacité de l'aide place, par définition, toute tentative de coordination dans le registre du bien. Il est moralement impossible de s'opposer à une volonté de coordination en soi, mais le leadership de la coordination devient un enjeu de taille au même titre que la compétition pour les fonds, la compétition pour la coordination débute. La coordination sera utilisée comme un palliatif pour mieux contrôler les acteurs. Cette compétition est exacerbée lorsque les acteurs n'ont pas de lien et ne s'identifient pas les uns aux autres comme la coordination avant 1997 qui était essentiellement limitée à des groupes d'acteurs restreints.

\section{$\underline{\text { Résume du processus de coordination }}$}

Il ne s'agit pas ici de dresser un inventaire non exhaustif des événements qui participent à son élaboration (on se reportera à cet effet à la chronologie ${ }^{13}$ ), mais de retracer les grandes lignes du processus afin de mieux en saisir les enjeux.

En juin 1996, l'UNOCHA organise une réunion informelle consultative sur la coordination de l'aide dans le but d'améliorer le processus de coordination de l'aide, de préparer un tableau global de tous les programmes d'assistance en Afghanistan, de mettre en place un mécanisme consultatif entre les différents participants et de revoir le mécanisme de l'Appel Consolidé. C'est là qu'est prise la décision d'organiser le futur forum d'Ashgabat. La conjonction de la volonté de réforme des Nations Unies (terrain et siège) et de la pression des donateurs fait de l'année 1997 le point initial.

L'objectif du forum est de permettre une rencontre des acteurs clefs de l'action humanitaire afin de définir une stratégie commune des méthodes et principes avec lesquels travailler, mais aussi attirer l'attention de l'opinion publique internationale sur l'Afghanistan par la présence de journalistes. C'est lors de ce forum que les responsables des Nations Unies affirment leur volonté de prendre la direction de l'aide en Afghanistan. Cela est confirmé en avril par la décision du comité administratif de coordination ACC, de choisir l'Afghanistan et le Mozambique ${ }^{14}$ comme pays pilote pour tester une approche globale des Nations Unies aux crises complexes (cadre stratégique).

Celle-ci est censée répondre aux besoins d'une meilleure coordination entre les différents acteurs. De leur côté les donateurs se constituent en groupe officiel : l'ASG (Afghan Support

\footnotetext{
${ }^{13}$ Voir Annexe ${ }^{\circ} 4$

${ }^{14}$ Le processus ne connaîtra pas de suite au Mozambique car il sera refusé par le gouvernement, en revanche un accord est en voie de signature avec la Sierra Leone.
} 
Group) groupe de soutien à l'Afghanistan, s'empressent d'affirmer leur soutien à la stratégie. Le groupe se donne pour mandat le suivi de la formulation. Le processus est lancé. Les liens informels qui permettaient aux différents acteurs de se rencontrer sont officialisés.

Cette année sera consacrée à la rédaction de la stratégie pour l'Afghanistan, des consultants venus de New York rédigent le texte qui- doit être soumis aux donateurs. La véritable mobilisation sur le terrain commence à l'automne lorsque les premiers brouillons sont soumis à commentaires. L'apparent consensus sur la nécessité de coordination trouve ses premières failles avec la déception à l'égard des textes de la stratégie, on parvient cependant à un accord sur un texte à soumettre aux donateurs, il sera approuvé lors de la deuxième réunion de l'ASG en décembre 1997.

Il semble au début de 1998 que tout le monde en soit arrivé au point où le PCP est certes une belle expression mais complètement vide de sens : rien n'est fait pour mettre en œuvre la stratégie. En dépit des avertissements des ONG et Agences onusiennes qui demandent un ralentissement du processus, les bailleurs de fonds font pression et demandent des résultats, l'Appel Consolidé ${ }^{13}$ élaboré en décembre est revu et publié en février. Une nouvelle présentation des projets, par secteurs ; est supposée refléter une meilleure coordination. Une équipe de consultants $^{16}$ constituée notamment de membres des départements des affaires politiques et humanitaires est sollicitée par UNOCHA afin d'élaborer des recommandations et un agenda pour mettre en œuvre le cadre stratégique. A nouveau, un ensemble de consultation est organisé afin de recueillir les avis des personnes de terrain. Le texte sort en avril : "Making a reality of Principled Common Programming, ${ }^{17}$. Il est censé refléter les vues des différents acteurs de l'aide, et apporter une réponse pratique aux problèmes et obstacles rencontrés en vue d'apporter une aide internationale coordonnée à Afghanistan.

Ce document décrit les principes sur lesquels seront basés l'assistance et propose une structure chargée d'assurer la coordination de la communauté d'aide et d'élaborer la programmation commune. Il en livre une définition : "common programming is a mechanism for establishing the assistance community's priorities, programmes and projects, based upon goals, principled and the express needs of Afghans. "

On note un changement dans la terminologie employée, on ne parle plus de "programme» mais de «programmation commune». Les différentes catégories d'acteurs lui offrent un soutien mitigé $^{18}$. De multiples débats sont organisés; ils deviennent rapidement orientés sur des questions de structures, (qui sera membre des organes, et surtout quel sera leur pouvoir de décision notamment en terme de sélection de projet ?). Les questions relatives au financement de la coordination sont aussi fréquemment soulevées. Les ONG soulignant le fait que si UNOCHA dispose de fonds à cet effet, cela représente pour elle une charge financière supplémentaire. En revanche, les interrogations de fond sur l'objectif de l'assistance internationale, les principes mettre en œuvre passent au second plan. Les débats sont éludés par des réponses vagues et consensuels.

Les premières structures voient le jour à partir de juin 1998 sans qu'il y ait de réelles appropriations par les acteurs et durant l'été les réunions en vue de la préparation de l'appel

${ }^{15}$ Le document existe depuis 1990, mais il n'est jusqu'à présent qu'une vision partielle de l'aide ; il ne reflète que le travail des UN, et omet presque totalement le travail entrepris par les O.N.G. à travers leur financement direct, elles n'y sont associées que depuis 1996.

${ }^{16}$ J. Mouchet UNHCR, Zarin Naqvi, world bank, j. Renninger UNDPA, Tom Tomsen Dacaar, Keating, Paula newberg.

${ }^{17} \mathrm{Cf}$. Annexe $\mathrm{n}^{\circ} 6$

${ }^{18}$ ces réactions ont été publiées par UNOCHA " responses to the making a reality document», Islamabad, 1998 
consolidé pour 1999 débutent. On assiste aux premiers échecs d'une tentative de coordination par le haut. L'appel constitue théoriquement un des outils indispensables de la programmation commune, puisque c'est en vue de sa publication que l'on cherche à définir les priorités pour le pays. Cependant ce processus est interrompu par les événements de l'été, tout d'abord en juillet l'expulsion des ONG de Kaboul pour avoir réfusé leur relogement forcé à Polytechnique, puis en août l'évacuation de l'ensemble du personnel expatrié suite aux bombardements américains sur Jalalabad.

Bien que le moment ne soit pas opportun, c'est l'occasion comme tous les acteurs sont rassemblés à Islamabad de raviver le processus afin de répondre aux pressions des donateurs, de préparer la constitution de l'A.P.B. (Afghan Programming body), organe de Programmation pour l'Afghanistan. La préparation de l'Appel est organisée à travers les structures nationales. Les ONG retournent en Afghanistan à partir d'octobre.

En 1999, des structures sont donc en place mais les fruits d'une meilleure coordination se font encore attendre. Les Nations Unies tentent de reprendre la direction d'un processus qui s'est peu a peu dilué en l'absence de personnel expatrié sur le terrain, le retour du personnel des Nations Unies annoncé depuis mars est très progressif et au début de l'été, peu sont déjà en place. Les ONG s'inquiètent des conséquences du Common Programming sur leur indépendance. En été, on tente à la fois de remettre en place les organes de coordination régionale (régional coordinating body RCB ) et nationale puis le processus s'accélère en vue de la sortie de l'Appel Consolidé 2000 pour l'Afghanistan. 


\section{DEUXIEME PARTIE : LA COORDINATION COMME ENJEU}

Il n'est pas possible d'analyser la manière dont le PCP a été pensé puis mis en œuvre sans en saisir les enjeux. La coordination, besoin naturel et évident sur une scène rendue si complexe par la multiplicité des acteurs et la divergence des intérêts, n'est pas un acte neutre. Elle répond certes à une nécessité ressentie par tous mais là s'arrête le consensus. Les objectifs avoués ou non des participants, individus et institutions entrent en contradiction, le débat comme la mise en œuvre du processus a été biaisé et l'objectif principal, l'amélioration de l'efficacité de l'action de la communauté d'aide pour l'Afghanistan est perdu de vue. Il ne s'agit pas ici de porter un jugement sur ces intérêts propres à chaque catégorie mais de comprendre qu'elles en sont les conséquences sur le processus.

\section{LE SYSTEME ONUSIEN}

Le processus de coordination est décrit par les acteurs comme étant une initiative onusienne, mais il convient de ne pas tomber dans le piège d'une substantivation de l'organisation et de discerner, en son sein, les différentes catégories d'acteurs qui n'ont pas loin s'en faut les mêmes motivations. Nous reprendrons pour la clarté de notre propos la distinction entre le siège et les agences sur le terrain. En effet elle permet de mieux cerner la complexité du double processus celui de cadre stratégique et de la programmation commune; et de mettre en lumière la variété des enjeux.

\subsection{Les difficultés au sein de l'institution}

On assiste au développement d'une réflexion au siège de l'ONU sur le rôle de l'institution face aux crises complexes.

\subsubsection{Les crises complexes : un nouveau défi}

La fin de la guerre froide a soulevé l'espoir que l'institution onusienne, libérée du frein de l'opposition Est-Ouest allait enfin pouvoir jouer un rôle en faveur de la paix et du développement. La résolution de certains conflits encourage cette vision optimiste de l'avenir. l'Afghanistan n'est pas en reste, la signature du traité de paix favorise la multiplication des projets de développement et d'aide au retour des réfugiés. Le moment semblait venu d'entreprendre la reconstruction du pays. Pourtant, les espérances sont rapidement déçues par l'évolution du conflit en combat interne entre factions. Le pays bascule peu à peu dans une crise complexe. ${ }^{19}$ Il répond en effet aux caractéristiques définies par A. Hallam ${ }^{20}$.

- «Intra-state rather than interstate conflict

- Difficulty in differentiation between civilians and combatants

- Violence directed towards civilians and civil structures

${ }^{19}$ complex emergency

${ }^{20}$ A. Hallam " evaluating humanitarian assistance programmes in Complex emergencies », Oxford, 1998 
- Fluidity of the situation on the ground

- Lack or absence of normal accountability mechanisms

- Potential and actual development of war economies

- Potential for humanitarian assistance to prolong the conflict

- A multiplicity of actors »

Or, l'évolution de la scène internationale, la fin de la guerre froide et la perte de l'intérêt stratégique, que représentaient pour les grandes puissances de nombreuses zones de conflit, laissent entre les mains des Nations Unies et des humanitaires le sort de nombreux pays et le règlement de ce type de crise. Les défis auxquels sont confrontés l'institution sont nombreux, le monde des années 1990 ne ressemble plus à celui dans lequel elle a été créée. Le nombre des acteurs privés intervenant dans les crises a augmenté et leurs actions ont pris de l'ampleur. L'intervention dans le cadre de conflits internes posent question quant au droit et responsabilités des Etats. La capacité de l'institution à traiter avec des autorités non reconnues dans des régions où les états se sont effondrés est loin d'être acquise.

Ces crises chroniques soulèvent des problématiques auxquelles aucune réponse définitive ne semble pouvoir être apportée. «Le nouvel ordre global» semble bien désordonné, et l'institution onusienne n'y est guère adaptée. L'espoir de son renouveau nait de la fin de la guerre froide cède rapidement le pas au constat de ces déficiences. Le désintérêt des grandes puissances lui laisse, certes, une plus grande marge de manœuvre mais elle ne sait guère la fructifier. L'institution n'est pas apte à assumer un tel leadership, tant elle ne semble avoir ni les moyens, ni (la volonté politique ?) de faire face à ses crises. Ce constat est corroboré par son incapacité à adopter une approche unifiée envers une crise. Comme nous l'avons vu la coordination entre le versant politique et humanitaire des activités des Nations Unies était jusqu'ici très limitée, les relations étant des relations de méfiance réciproque.

\subsubsection{Urgence développement : la difficile transition}

Dans le domaine conceptuel, la lassitude envers ces crises chroniques (!), amène au développement de réflexion sur la prévention, sur la transition entre action d'urgence et de développement et sur la façon dont l'assistance peut contribuer à la construction de la paix. Cette division classique dans l'analyse conceptuelle de la situation, se révèle inopérante dans des situations aussi complexes que l'Afghanistan où le conflit perdure et où chacun s'accorde pour dire que l'on ne peut attendre la paix pour mettre en ouvre des activités de développement. Le concept de zone grise entre l'urgence et le développement fait fortune, il souligne l'impasse dans laquelle se situe une réflexion qui ne parvient pas à dépasser les schémas habituels. La remise en cause de cette ligne de fracture participe de la tentative d'apporter une réponse aux crises complexes. L'échec des tentatives politiques d'apporter la paix impose la recherche d'autres alternatives. Les causes de la crise ne sont plus identifiées comme étant seulement politiques. Elles s'enracinent dans l'absence d'alternative au combat pour des générations n'ayant connu que la guerre, dans la faiblesse de la société civile. Or les réponses à ces défaillances doivent être apportées par les acteurs de l'aide et non plus par des négociations politiques. Ce type d'analyse est certes pertinent mais il nous semble dangereux lorsqu'il permet, comme c'est le cas, en Afghanistan de décharger les politiques de leurs responsabilités pour les remettre entre les mains de la communauté d'assistance. Certes le conflit oppose des factions internes mais il est nourri par des interventions extérieures, les agents de l'aide ne peuvent seuls en éliminer les causes. 


\subsubsection{La crise financière}

La perte de crédibilité de l'ONU est d'autant plus grave qu'elle entraîne une crise financière. Les activités humanitaires et de développement dépendent essentiellement des contributions volontaires des Etats. Non seulement ils peuvent choisir les projets auxquels ils contribuent mais aussi influer sur les politiques des agences. Mais, il n'existe pas de coordination entre les représentants des Etats membres qui présentent leurs doléances aux agences de manière dispersée. Les contributions obligatoires sont l'objet de retard de paiement, voire d'imposition de condition par les Etats membres, nous ne reviendrons pas ici sur l'exemple illustre des Etats Unis. Ainsi, les programmes sont largement orientés par les Etats membres. Les intérêts qu'ils défendent ne sont pas dénués de considérations politiques. Les Nations Unies constituent un canal de transmission des volontés de ses membres. La structure hiérarchique de l'institution garantit que les politiques définies au siège ne seront pas biaisées au niveau du terrain.

\subsubsection{Les querelles internes à l'organisation}

Si nous continuons à employer le vocable système pour la définir, l'institution est loin d'en avoir les propriétés. Elle s'est, au cours du demi-siècle, développée. De nouvelles agences ont été créées, cette expansion n'a pas toujours eu lieu de manière ordonnée, les limites des mandats respectifs des agences étant confus. On constate, soit des duplications de fonction, soit l'apparition de nouveaux problèmes n'entrant pas de le cadre établi. Tel est le cas des personnes déplacées dont la prise en charge a été l'objet de longues discussions car elle ne figurait au mandat d'aucune des agences. Au contraire, elles entreprennent parfois des programmes qui outrepassent leur mandat afin de s'assurer des financements. Ainsi, le HCR a mis en œuvre en Afghanistan des programmes de rapatriement tentant de prendre en charge les personnes rapatriées après leur retour alors qu'il n'en a ni le mandat ni les capacités opérationnelles. Les chefs des sièges ne sont responsables devant personne ${ }^{21}$. Loin des réalités du terrain ils imposent parfois des politiques que les fonctionnaires détachés désapprouvent mais ne parviennent pas toujours à influencer. Ainsi, le siège du PAM a refusé de mettre en œuvre des programmes de denrées alimentaires contre travail« Food for work" en Afghanistan car ceux-ci ne bénéficiaient pas également aux femmes. Les représentants de l'organisation à Islamabad étaient depuis longtemps conscients de l'ineptie de cette mesure, qui prive les femmes comme les hommes de l'assistance, mais ils n'ont obtenu des dérogations qu'après deux ans de négociations avec leur siège.

La fracture est aggravée en Afghanistan par l'indépendance qu'ont pris les agences techniques les unes à l'égard des autres, indépendance jalousement protégée. Chacun fait référence à son mandat propre et spécifique pour se parer de toute interférence extérieure dans ses programmes et budget. Or, la frontière avec l'extérieur commence pour certains au portail du bureau de l'agence. Dans certains cas le siège de l'agence, et d'autant plus celui des Nations Unies, ne conserve aucun contrôle. Ainsi l'OMS a un fonctionnement particulier, rattachée au bureau régional d'Alexandrie, son siège n'a aucun pouvoir de contrôle sur ses activités. Nous l'avons vu précédemment, les agences sont contrôlées en ce qui concerne les décisions stratégiques mais il existe au niveau local des espaces de liberté qui permettent d'échapper à cette tutelle. Les agences ont parfois été noyautées par des réseaux puissants, certaines présentent un fort degré de corruption, ce phénomène connu de tous n'a jamais été étudié en profondeur car c'est un sujet sensible. Or, le système onusien montré du doigt pour son inefficacité ne peut

${ }^{21}$ si ce n'est leur " executive board » auquel ils appartiennent eux-mêmes. M. Bertrand ; "The United Nations ; Past, present and future ", p80, Kluwer Law International, la hague, 1997 
tolérer de tels écarts, d'autant plus que les faits de corruption s'ils ne sont jamais révélés au grand jour minent la confiance en l'institution. Outre la pénétration des agences par des réseaux locaux, elles font face à un grave problème de recrutement de personnel international. «L'Afghanistan c'était le bagne des Nations Unies, il était très difficile de trouver du personnel de qualité pour ce pays ${ }^{22}$.

\subsection{La nécessaire réforme des Nations Unies}

\subsubsection{La réforme de 1997}

Il apparaît donc impératif de réformer le système dans son ensemble, afin de répondre aux multiples attaques dont l'organisation est l'objet. La réflexion, engagée à New York depuis 1995, sur le besoin de réforme des Nations Unies aux crises complexes est l'occasion de tenter de répondre à un des problèmes fondamentaux du système : l'hypertrophie des agences qu'elles ne contrôlent plus. Cette réflexion est remise à l'ordre du jour avec l'arrivée de Kofi Annam à la tête du secrétariat général en 1997. Cependant, peu de progrès ont été accomplis dans les années 90 et l'année 1997 voit une accélération du processus. A l'occasion de la réforme de 1997, des réarrangements internes ont été apportés.

Le département des affaires humanitaires (DHA) est réorganisé. Son rôle consistait en la coordination de l'aide humanitaire au sein du système onusien. Il conseille le secrétaire général sur les affaires humanitaires. Fort critiqué, il était d'une efficacité limitée. Il devait faire face au mécontentement de certaines agences à l'égard de son trop grand rôle opérationnel, il était perçu comme un concurrent. Nombreux, parmi le personnel des agences, étaient davantage favorables, à l'idée d'une agence leader plutôt qu'à la mise en place d'un organe supplémentaire en vue de les coordonner. C'est le cas notamment au sein du HCR ou de l'UNICEF, le premier ayant été nommé agence leader pour les opérations en ex-Yougoslavie et au Rwanda ${ }^{23}$. Le département est renommé OCHA (Office for the Coordination of Humanitarian Affairs) et le volet opérationnel de son action est remis à d'autres agences. Il a trois fonctions

1. «Fonction de coordination afin de s'assurer que toutes les questions humanitaires y compris celles échappant aux mandats actuels des agences (telles que les personnes déplacées) soient prises en charge

2. Faire du lobbying auprès des organes politiques notamment le conseil de sécurité

3. Coordination des actions humanitaires d'urgence. »

Un nouvel organe, le comité exécutif pour les affaires humanitaires est formé, (ECHA) ${ }^{24}$ est supposé permettre un plus grand lien entre les organes politiques de développement et de droit de l'homme.

Le Département des Affaires Politiques dépendant du Secrétaire Général est chargé de l'analyse du contexte politique des actions humanitaires, il devient à l'occasion de la réforme de 1997 le point central de la réflexion sur l'après conflit et la construction de la paix.. Les droits de l'homme sont proclamés thèmes transversaux pour tous les champs d'action du secrétariat.

\footnotetext{
${ }^{22}$ Victor Carvell , counsellor development, ambassade du Canada

23 Arve Ofstad dir. "The UN and International organisations as channels for Danish humanitarian assistance 1992-98 » Draft final report 1999, Chr.Michelsen institute, Bergen

${ }^{24}$ Il établit en juin 1997 une recommandation concernant la question de la femme
} 


\subsubsection{L'Elaboration du Cadre Stratégique (Strategic Framework)}

\section{- Le choix de l'Afghanistan comme pays pilote}

Outre les remaniements administratifs, on note une accélération de la réflexion sur les crises complexes. C'est dans ce cadre qu'une attention toute particulière sera accordée à l'Afghanistan. Deux organes auront une place critique dans la décision d'élaborer une nouvelle stratégie des Nations Unies pour aborder les pays en crise complexe: ACC (Comité Administratif de Coordination) $^{25}$ et CPQCP (Comité Consultatif sur les Programmes et Questions Opérationnelles). Dans un rapport ${ }^{26}$ au CPQCP lors de sa dixième session à Genève en mars 1997, Hugh Cholmondeley dresse le constat de l'inadaptation des réponses des Nations Unies aux crises complexes. L'Afghanistan fait partie des 25 pays identifiés comme ayant vécu des crises majeures et sur lesquels l'attention doit être portée. Le rapport conclut à la nécessité d'une meilleure coordination entre les différents acteurs. Afin de répondre à ce besoin et de jeter les bases du développement, il est préconisé d'élaborer un cadre stratégique à deux volets : d'une part une stratégie de négociations politiques et d'autre part un cadre stratégique pour le développement (strategic framework for recovery) Le cadre stratégique offre un espace pour une meilleure harmonie dans la programmation et le financement de l'aide. Il passe par une analyse de l'environnement interne du pays et doit permettre une approche intégrée cohérente et unifiée, la planification des réponses aux urgences et faciliter le développement. Concernant ce second point, le rapport constate le lien entre les phases d'urgence et de développement.

Le comité recommande la sélection de deux pays test pour mettre en œuvre le cadre stratégique et définit les critères de sélection de ces pays :

- crises prolongées, conditions d'urgence et poches d'instabilité,

- pays étant en poste crise ou émergeant de la phase la plus pointue de la crise

Ceci constitue la première étape du processus que nous allons analyser. C'est la confirmation de la volonté d'établir un cadre stratégique pour aborder les pays en crise et de l'intérêt de l'institution au niveau du siège pour ces questions. Un mois plus tard, en avril 97, le comité administratif de coordination choisit l'Afghanistan et le Mozambique comme pays pilote. Deux raisons ont été avancées pour justifier la désignation de l'Afghanistan comme pays test :

- le processus y est déjà en cours. En effet le forum d'Ashgabat a été récemment organisé et des mécanismes de coordination et de dialogue existent tels que le Groupe de Travail sur l'Afghanistan (ATF Afghan Task Force)

- C'est un moyen d'attirer l'attention de l'opinion publique internationale sur des crises oubliées. En effet, l'assistance internationale se trouve confrontée à une baisse des financements et à une soi-disant lassitude des donateurs.

Il semble en outre que la pression de Sadako Osata, haut commissaire pour les réfugiés, a été décisive dans le choix l'Afghanistan. N'oublions pas que les Afghans représentent la plus grande population réfugiée au monde.

\footnotetext{
${ }^{25}$ Le plus important organe de coordination onusien, composé du Secrétaire général, des têtes des secrétariats des agences spécialisées.

${ }^{26}$ Peace principles partnership and programmes», the role of the UN system in response to crisis and recovery, astrategic approach ; Hugh Chomondeley 1997
} 
Les raisons qui ont motivé le choix de l'Afghanistan ne sont certainement pas uniquement liées à l'analyse de la situation sur le terrain. Il ne s'agit pas seulement pour les décideurs de mettre en œuvre le cadre stratégique mais aussi de reprendre en main les agences qui tendent à prendre trop d'autonomie. Le mandat officieux d'Alfredo Witschi-Cestari, nommé coordinateur humanitaire résident en 1996, était précisément de «restructurer et épurer le système. 》

En outre, il paraissait plus facile d'entreprendre une réforme dans un contexte où les intérêts géopolitiques des états étaient moindres ou du moins n'entrent pas en conflit, afin d'éviter les écueils d'un contexte où toute réforme technique aurait été freinée par des enjeux politiques. L'Afghanistan présente à cet égard un avantage de taille, la communauté internationale s'accorde quasiment dans sa totalité pour ne pas reconnaître l'autorité Taleb. L'absence de gouvernement reconnu permet aux Nations Unies de se présenter comme la seule institution légitime en vue d'une meilleure coordination. La seule aussi dotée des moyens financiers d'une telle action.

Cependant, la pertinence d'un tel choix peut être questionnée : la multitude d'acteurs présents voire les antagonismes qui règnent entre eux font du pays un cas certes intéressant pour une tentative de meilleure coordination mais difficile. Ce choix dénote une méconnaissance du terrain. Le fait que les acteurs de l'aide en Afghanistan sont présents depuis des années, que les interactions entre acteurs sont le fruit d'une histoire complexe faite de mauvaise communication, de compétition, qu'il n'existe pas de confiance mutuelle, et que la volonté de collaborer est loin d'être acquise, n'ont pas été pris en compte. On se propose de plaquer une nouvelle solution, de proposer une nouvelle démarche sans au préalable identifier les particularités et les obstacles sur le terrain à l'éventuelle mise en cuvre d'une approche globale.

Certes, tout le monde s'accorde sur la nécessité de sortir de la dichotomie aide d'urgence/ développement, sur le fait que presque 20 ans d'aide n'ont pas permis d'apporter de solution définitive à la crise afghane ; mais il existe aussi des obstacles quasi structurels à une véritable collaboration sur le terrain dont on ne semble guère se préoccuper. On cherche à développer une nouvelle stratégie en brûlant l'étape indispensable d'une analyse en profondeur du système en place.

Un autre écueil fondamental de cette approche est qu'elle repose uniquement sur l'amélioration du dialogue et de la coordination entre les acteurs. Nul doute que ceci constitue une condition nécessaire à la résolution des crises mais cette condition n'est certainement pas suffisante. Il n'existe aucun substitut aux indispensables réformes internes à chaque organisation, faire croire que la coordination permettra de pallier les dysfonctionnements identifiés relève de l'utopie ou du manque de courage politique. En effet, les freins à une réforme en profondeur du système onusien viennent autant de la lourdeur de sa bureaucratie que du manque de volonté des états membres. Si leurs représentants ne sont pas avares de discours sur le besoin de réforme, ils ne lui en laissent pas les moyens.

Ce processus, s'il prétend s'appuyer sur les acteurs présents sur le terrain et prendre en compte les avis de l'ensemble de la communauté de l'aide, reste dans ces premières phases dirigé et contrôlé par le siège de l'organisation. Deux équipes chargées de l'élaboration des textes fondateurs sont envoyées en mission, mais pour des durées très limitées, elles ne sont composées que de personnel basé à New York et chacune ne compte qu'un représentant des ONG. 
Après une consultation qui déçoit les acteurs de terrain (ONG comme agences des Nations Unies), un premier texte est refusé par le Groupe de Travail sur l'Afghanistan (ATF) car il reste beaucoup trop décalé des réalités du terrain. La consultation semble n'avoir été qu'une simple formalité. Ainsi, il recommande une multiplication par 4 du volume de l'aide et la création de 500.000 emplois par an. C'est cette seconde recommandation qui avait fait bondir les acteurs de terrain, par son caractère irréaliste mais qui a été maintenue. Le texte ne se base pas sur l'analyse des potentialités de la communauté internationale pour répondre aux différents problèmes. Il ferme les yeux sur le fait que l'aide est par définition restreinte au plan financier et que tous les besoins ne sauraient être satisfaits. Au-delà, il occulte complètement les limites en terme de ressources humaines et de capacités opérationnelles. Cette analyse basée sur les besoins n'est pas pertinente. En effet au niveau local, il est certes important de se fonder sur les besoins des communautés, de veiller à ne pas apporter des solutions plaquées de l'extérieur qui détruisent les équilibres socio-économiques. En revanche, une approche globale; destinée à élaborer une stratégie en vue du développement du pays; ne peut se baser sur les besoins qui sont, par définition, incommensurables dans un pays qui n'est pas sorti de 20 ans de guerre. Cela ne peut que mener à des aberrations de ce type.

Le texte recommande que la coordination repose sur une approche plus directrice, ce qui provoque évidemment un tollé des ONG comme des agences des Nations Unies. Les secondes, répugnant à travailler en partenariat avec les autres acteurs, arguent du fait qu'elles doivent se conformer à la politique de leur propre siège. Pour les rédacteurs du cadre stratégique, le groupe de travail sur l'Afghanistan (l'Afghan Task Force) et le coordinateur des Nations Unies sont là en attendant que des autorités légitimes émergent. Il s'agit ainsi de combler un vide qu'une institution telle que les Nations Unies ne peut supporter: l'absence d'une autorité, d'un gouvernement légitime. En effet, les Nations Unies, organisation d'Etats, ayant pour mandat de travailler avec des Etats, se trouvent fort dépourvus quand un pays n'est point pourvu de gouvernement reconnu. L'Afghanistan qui fait partie des trois pays au monde, sans Etat reconnu, représente pour l'institution un défi, voire une menace auxquels elle ne sait comment faire face Elle fonde son action sur le droit international qui est un droit d'état à état, elle a beau jeu d'opposer aux Taleban des conventions telles que celles des droits de l'enfant, qui ont été signées auparavant par l'Etat Afghan. Quant au caractère légitime du gouvernement tant souhaité, il s'agit évidemment de reconnaissance par les autres Etats et non de la légitimité du peuple dont on a vu ailleurs que les Nations Unies se soucient moins.

Un second texte proposé par UNOCHA est finalement adopté : « la Stratégie d'assistance à l'Afghanistan ${ }^{27}{ }^{27}$

« Ce texte fixe 3 grands objectifs à l'Aide Internationale :

- au minimum ne pas aggraver le conflit en cours et, là où cela sera possible, favoriser la mise en place de conditions favorables à une paix durable,

- aider les afghans dans la création de conditions et de moyens d'existence meilleurs (notion de « livelihoods »),

- sauver des vies et réduire les souffrances humaines

Ce texte définit aussi les grands principes sur lesquels doit s'appuyer l'Aide Internationale : droits de l'Homme, égalité des sexes, pas de discrimination, souveraineté afghane, participation des communautés, transparence, contrôle des cultures illicites, monitoring et évaluation indépendants.

${ }^{27}$ JF Cautain «note sur l'évolution du système de l'aide », MADERA, Note interne,Peshawar, 1998 
Il jette aussi les bases d'un programme commun pour l'Afghanistan qui doit être le résultat d'un processus de large concertation tenant compte des réalités du terrain et dans lequel l'ensemble des actions des différents acteurs locaux (agences des Nations Unies et ONG) devra s'intégrer.

Enfin, il lance l'idée d'un fonds unique pour l'Aide en Afghanistan par lequel transiterait l'ensemble des subventions destinées à ce pays et qui serait géré par les Nations Unies. »

Ce texte sera adopté par les donateurs qui repousseront cependant l'idée de fonds commun. Il a la pâleur des textes consensuels mais servira de base pour la rédaction du cadre stratégique, dont la version définitive signée par la sous-secrétaire générale sera adoptée en août $1998^{28}$. En effet, elle dut prendre en charge cette initiative afin de lui donner plus de poids et de parvenir à l'imposer aux agences.

Il s'agit de ne pas négliger cette implication du siège de l'institution; si l'accent est mis à l'envi par les personnes interrogées sur le caractère terrain de l'initiative, elle est le fruit de pressions extérieures, elle est part d'un effort global de réforme de l'institution onusienne. Loin d'être une réforme purement opérationnelle et donc neutre, elle est l'objet de luttes de pouvoir en son sein. Ce facteur explique en partie la très faible part des acteurs de terrain qui se sentent concernés et qui s'impliquent dans la réforme. Il semble que le degré d'intérêt soit inversement proportionnel à la distance par rapport au terrain. Si on trouve encore à Islamabad des personnes qui s'investissent elles se font beaucoup plus rares en Afghanistan ou à Peshawar, lorsque l'existence même du processus n'est pas complètement ignorée.

La concurrence entre agences fait de la réforme un sujet délicat si le Secrétariat Général s'y intéresse de près et ne souhaite pas abandonner les efforts en ce sens. Il demeure méfiant envers ce processus qui n'a pas la faveur des chefs des agences or il ne peut se permettre de s'opposer à eux. Ils se font les gardiens jaloux de l'indépendance notamment financière de leur institution et sont loin d'être acquis à ce type de coordination. Cependant, cette réflexion sur le cadre stratégique est, outre l'expression de la volonté d'apporter une réponse mieux appropriée aux nouveaux défis, celle de la nécessité pour le siège des Nations Unies de reprendre en main les agences à travers l'UNOCHA.

\subsection{Les tentatives de coordination depuis Islamabad}

\subsubsection{Le poids de l'UNOCHA}

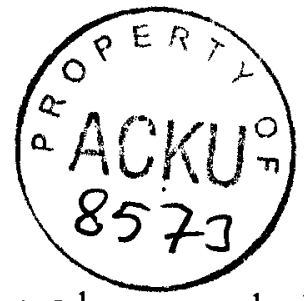

La réflexion sur la nécessité d'une meilleure coordination n'est pas le monopole du siège. Elle se poursuit aussi à Islamabad et elle y est antérieure à la reforme de 97 . Mais comme nous l'avons vu dans la première partie, les différentes tentatives de coordination mises en cuvre dans les années 90 n'ont pas apporté de solution satisfaisante aux problèmes, l'UNOCHA ne parvenant pas à remplir cette fonction. Les pressions conjuguées de certains bailleurs de fonds poussent l'UNOCHA à prendre des initiatives en 1996.

La nécessité de justifier sa présence et les fonds qui sont octroyés est un important facteur de mobilisation. La critique latente des donateurs sur l'incapacité du système à apporter une réponse à une crise qui dure, s'accompagne d'une baisse des fonds versés au titre de l'aide aux

\footnotetext{
${ }^{28}$ Cf.Annexe $n^{\circ} 7$
} 
Nations Unies, à laquelle il est indispensable de remédier pour assurer la survie de certaines agences, tel l'UNDP qui se trouve dans une position financière critique. Il est à l'origine d'une tentative de coordination des projets des agences des Nations Unies travaillant dans le domaine du développement, l'initiative PEACE (Poverty Eradication and Community empowerment). Cette initiative s'est soldée par un échec, lié-en partie à une divergence d'approche entre les stratégies de la FAO en zones rurales et celles d'Habitat en zone urbaines. Mais les financements ne sont pas assurés pour l'année 2000, et ses promoteurs en sont aujourd'hui réduits à s'interroger sur la façon de conserver leur emploi, alors que ce programme risque de s'arrêter.

C'est en juin 1996 que l'UNOCHA propose d'engager une grande réflexion sur l'aide internationale en Afghanistan. Lors d'une réunion consultative réunissant les trois groupes d'acteurs, que la décision est prise de créer une Force de Travail entre les agences (Inter Agency Task Force ${ }^{29}$ ), pour préparer l'Appel 1997 et un grand forum dont le but sera notamment d'examiner la conformité des programmes d'aide aux normes internationales et standards des droits de l'homme. L'organisation du forum qui aura lieu à Ashgabat (Turkmenistan) en janvier 1997 est pris en charge par UNOCHA. Ce forum a rassemblé l'ensemble des acteurs de l'aide en Afghanistan ainsi que des journalistes, des chercheurs et les autorités afghanes. C'est lors de ce forum que les Nations Unies via UNOCHA ont annoncé publiquement leur volonté de prendre la direction de l'aide en Afghanistan. Cette déclaration n'a pas entraîné dans un premier temps de grandes protestations de la part des O.N.G.

Les principaux débats soulevés durant cette rencontre, seront l'objet de discussions tout au long du processus.

- Lien urgence /développement ; aide internationale /construction de la paix

- Lien aide internationale et politique

- L'aide ne peut pas se substituer à l'action politique internationale

- Nécessite d'une meilleure coordination entre différentes catégories d'acteurs et au niveau interne à chaque groupe nécessité d'une meilleure efficacité, problème de compétition, transparence.

- Principes d'opposition entre ceux qui sont pour des positions de principes stricts/ ceux qui demandent de faire attention à ne pas imposer des principes qui risquent d 'être perçu comme occidentaux.

Ce forum a permis de soulever de nombreux débats clefs mais pas d'obtenir un consensus. Cependant, la décision est prise d'élaborer une stratégie globale de l'aide en Afghanistan dans les 6 mois à venir et de fusionner les deux fonctions de coordination du système des Nations Unies, UNOCHA et UNDP. Cette fusion sera effective le 15 mars 1997, en revanche les deux organismes conservent des administrations et bureaux séparés.

Ce processus représente un enjeu particulièrement important pour l'UNOCHA car c'est la définition même de son rôle qui en dépend. Il se doit de faire preuve de sa capacité à coordonner l'ensemble des acteurs.

\subsubsection{La réticence des agences.}

Les habitudes d'indépendance des agences s'accommodent mal de cette volonté de reprendre en main l'ensemble du paysage de l'aide en Afghanistan, d'autant plus que cette

\footnotetext{
${ }^{29}$ Future Afghan Task Force (force de travail pour l'Afghanistan), elle est composée de représentants des Agences des Nations Unies, des coordinations d'ONG et de donateurs.
} 
volonté s'affirme comme la fin d'une ère. "La coordination par le consensus avec un coordinateur agissant comme facilitateur est clairement inefficace. Un commandement beaucoup plus fort et une approche plus robuste et globale est nécessaire. ${ }^{30}$. Les réactions des agences aux différents textes proposés sont donc fort mitigées. Les principales critiques viennent des personnels présents sur le terrain qui expriment leurs doutes quant à l'efficacité de cette initiative. Le personnel Afghan y est peu ou pas sensibilisé. C'est seulement en mai 1998 que les différentes agences ont affirmé leur volonté de s'impliquer dans le processus sous la pression du ministre hollandais du développement $M$. Pronk, lors de la réunion du groupe de soutien à l'Afghanistan à Londres. Mais cet accord officiel des dirigeants des agences ne signifie en rien une réelle implication de l'ensemble des personnes. Les réactions des personnes interrogées relèvent soit de la critique acerbe : «le Common Programming : je n'ai pas envie de perdre mon temps avec ça, ils veulent établir un pouvoir central qui coordonne tout alors que ce sont les affinités naturelles qui font avancer » à un soutien affirmé : «il faut que nous réussissions cette année, sinon tout est perdu ». Cette dernière remarque vient d'une personne de l'UNDP dont on sait qu'il doit faire la preuve d'une meilleure efficacité s'il veut regagner la confiance des donateurs.

Si l'enjeu est de taille pour les Nations Unies dans leur ensemble, il n'est pas négligeable non plus pour les individus au sein même du système. Il convient de ne pas les soustraire à l'analyse. Lors des débuts du processus il s'agissait essentiellement de consultants détachés par d'autres départements, mais le nombre d'employés directement liés au Common Programming a augmenté; or les personnes embauchées par UNOCHA ne sont pas fonctionnaires internationaux mais ont des contrats à court terme et doivent donc impérativement faire preuve de résultat. Ainsi, pour certains, c'est leur carrière qui est en jeu, ce d'autant plus que ce qui se passe en Afghanistan intéresse les sièges de New York et Genève au plus haut point. Dans cette perspective, il ne s'agit pas de prendre le temps d'une longue réflexion et la consultation des points de vue de chacun, qui ne peut que contredire les beaux schémas élaborés loin des réalités de terrain, n'est qu'un frein. L'important n'est pas de favoriser un meilleur dialogue mais de faire croire qu'il existe, afin de montrer que l'on obtient des résultats et de justifier son poste et une éventuelle promotion.

\section{LA PRESSION DES DONATEURS}

\section{$\underline{\text { 2.1. Leur place dans le processus }}$}

Je traiterais ici principalement de la place des représentants sur le terrain des différents états investis en Afghanistan. Ils sont, en effet, les interlocuteurs directs des agences des Nations Unies et des ONG pour les thèmes concernant la coordination. En revanche, lors des réunions bi-annuelles de l'ASG, sont présents des fonctionnaires et personnalités politiques des capitales. Il existe cependant une exception de taille à cette règle, c'est l'influence de $\mathrm{M}$. Pronk alors ministre du développement des Pays Bas. Celui-ci a joué un rôle considérable puisque c'est lui qui a fait pression sur UNOCHA pour que soit lancé le forum d'Ashgabat et impulsé la coordination.

En dépit de leurs intérêts divergents, les bailleurs de fonds jouent donc, depuis le début du processus, un rôle important de catalyseur. Ils font pression et exigent des progrès et une amélioration de l'efficacité de l'aide. Ils suivent de près l'évolution ou tentent de le faire. En

\footnotetext{
${ }^{30}$ Donini, "Afghanistan, coordination in a fragmented state", p25, DHA, New York, 1996,
} 
effet, ils sont trop éloignés du terrain pour avoir une réelle idée de ce qui s'y passe. Les donateurs se sont dotés des outils nécessaires afin de mieux suivre le processus. Dès avril 97 lors de sa première réunion à Genève, le Groupe de soutien à l'Afghanistan (ASG) se donne pour mandat de suivre les progrès de formulation du cadre stratégique. Pour les donateurs, il s'agit non seulement de se tenir informés mais surtout d'influencer les débats. Et si une fois encore l'objectif d'efficacité est mis en avant, il semble que ce ne soit pas la seule motivation. Ils ont toujours été les destinataires finaux des grands textes préparatoires. Le cadre stratégique comme le « Making a reality of PCP » n'ont été définitivement adoptés qu'après approbation du Groupe de Soutien. Mais, ce n'est pas seulement un statut passif d'observateurs donnant leur approbation que s'octroient les donateurs, il s'agit pour eux de prendre une part active au processus et l'on peut dire aujourd'hui qu'ils en sont les chefs d'orchestre. De fait, ils insistent pour que les acteurs de l'aide définissent des priorités parmi les projets qui leur sont soumis à travers l'Appel Consolidé, qui sera étudié plus loin.

Les points de vue collectifs et individuels montrent par leur divergence la complexité des enjeux. L'ensemble des donateurs expriment leur soutien pour le processus de coordination, les propos sont au premier abord uniformes, aucun n'ose aller à l'encontre du discours ambiant sur la coordination. En revanche, des entretiens plus approfondis montre que le degré d'implication n'est pas toujours aussi important. Les petits pays donateurs tel que la Finlande, le Japon ou la France montre un intérêt voire des connaissances limitées. L'Union Européenne met en exergue les défauts du processus. En revanche les Etats Unis, le Royaume Uni, les Pays Bas, font de l'inscription des projets dans l'Appel Consolidé une condition de financement ; le Canada actuellement à la présidence de l'ASG exprime un soutien actif confiant au processus.

\subsection{Leurs objectifs}

Les objectifs des bailleurs de fonds ont une influence sur les grandes orientations du processus en raison de leur position de financier et de leur implication. Les acteurs de l'aide et les responsables de la coordination s'interrogent en permanence sur les motivations des bailleurs de fonds. Il semble que les messages que ceux-ci émettent ne soient pas toujours compris et lors des débats de fonds sur l'avenir du PCP revient sans cesse la question de savoir quelles sont les positions et les attentes des donateurs. Il est étonnant à cet égard de constater que la réflexion est rarement inversée, les acteurs de l'aide semble avoir pour beaucoup perdu confiance en leur capacité de dialoguer avec les donateurs et de leur faire part de l'expérience de terrain. Ils adoptent davantage une attitude de réaction que de proposition, ils estiment ne pas être écoutés, individuellement ils ne prennent pas suffisamment de temps pour expliquer leurs points de vue et collectivement ils s'inclinent devant le pouvoir financier. Le caractère confus des messages qui proviennent des bailleurs de fonds est lié à deux facteurs; d'une part ils n'ont pas tous les mêmes objectifs et d'autre part ils n'ont pas toujours la marge de manœuvre nécessaire pour imposer leur vue à leur capitale.

Les Hollandais, qui ont donné l'impulsion initiatrice avaient trois objectifs majeurs :

- renforcer la coopération tout d'abord entre les différents acteurs de l'aide afin d'éviter notamment les duplications de projets, ensuite entre le département des affaires politiques et humanitaires des Nations Unies,

- le second objectif était de passer d'une assistance essentiellement d'urgence à des projets à plus long terme afin de participer à la construction de la paix,

- enfin ils voulaient encourager l'afghanisation de l'aide. 
Pour les Canadiens, l'objectif principal est de promouvoir une meilleure coordination à l'intérieur du système onusien, de les forcer à intervenir sur la base de principes. Le représentant canadien déclare que les Nations Unies ont beaucoup moins progressé que les ONG durant les années 90 . Il fustige les attitudes du personnel onusien : «people were protecting their job and mandate». Le mécontentement des représentants des Etats envers les agences des Nations Unies est, pour lui, le principal facteur qui a poussé à cette initiative dont le but est d'améliorer le fonctionnement onusien. Il est beaucoup plus confiant envers les grandes ONG internationales «nous savions que nous pouvons travailler avec elles», en ce qui les concerne il souhaite essentiellement promouvoir une meilleure diffusion de l'information; enfin la dernière attente à l'égard du PCP est qu'il permette de résoudre le problème des multiples contractants afghans qui se présentent comme des ONG mais qui font face à un crucial déficit de compétence en matière de gestion comme d'approche du développement.

Ainsi la majeure partie des donateurs attend de ce processus qu'il offre l'opportunité à travers une meilleure coordination, d'une aide plus efficace et dispensée à un moindre coût. En outre, ils ne jouissent pas toujours ni d'une réelle connaissance du terrain ni des moyens nécessaires pour l'acquérir. Si certains sont présents depuis de nombreuses années, d'autres ne restent pas en poste suffisamment longtemps pour prétendre connaître le terrain d'autant plus qu'ils n'ont pas tous la possibilité de voyager dans le pays. Cependant, quelques représentants sont en poste à Islamabad ou Peshawar depuis suffisamment longtemps pour avoir une bonne connaissance des différents acteurs et des problématiques.

Ils expriment le souhait quasi unanime que les acteurs de l'aide établissent eux-mêmes une procédure permettant de définir les grandes orientations de la communauté internationale en Afghanistan. Ils attendent à la fois une réduction des duplications, des chevauchements de projet et une couverture sectorielle et géographique de l'aide comblant les lacunes actuelles. Le second thème fondamental est de définir parmi la multitude des projets qui leur sont soumis ceux qui répondent aux priorités. Comme le déclare le représentant suédois ${ }^{31}$, "la définition de priorité sera le moyen pour moi de prendre des décisions mieux adaptées, nous ne connaissons pas assez l'Afghanistan ". Mais là aussi s'arrête le consensus. Pour le Canadien c'est la définition de priorité parmi les projets des agences des Nations Unies qui est essentielle et c'est donc l'UNOCHA qui doit en être responsable, pour d'autres c'est l'ensemble des projets qui doivent être pris en compte et le caractère consultatif du processus est fondamental. Le représentant français, s'il exprime son soutien à l'idée de définition de priorités communes, craint que « la gestion de l'aide ne soit monopolisée par un petit groupe de donateurs ou par les Nations Unies ». Il convient de préciser que la France a une position particulièrement critique à l'égard du PCP. Le représentant de l'Union Européenne à Peshawar, M. Mac Leod remet lui en cause l'idée même de définir des priorités, il affirme que cela ne «signifie pas grand chose, car ce qu'il faut c'est identifier les actions possibles à court, moyen et long terme». Ainsi «l'appel annuel ne peut être une réponse fiable et viable aux défis de l'Afghanistan, il représente beaucoup de travail pour peu de résultat $»$.

Les différents bailleurs de fonds soutenant le processus semblent en revanche s'accorder sur le fait qu'au niveau global, l'UNOCHA doit en être le leader : " nous les payons pour cela, il doivent faire leur travail ». Mais ils affichent aussi la volonté de voir les ONG prendre une part active au niveau des différents organes et des coordinations régionales.

\footnotetext{
${ }^{31}$ entretien avec Michael Lindvall, ambassade de Suède.
} 
On comprend ici la schizophrénie des représentants des bailleurs de fonds partagés entre leur expérience propre du terrain et les politiques de leur pays, leur approche des problèmes et leur mandat de représentant d'un état. Celle-ci se ressent à deux niveaux. Tout d'abord, ils expriment un soutien naturel aux Nations Unies, émanation des états mais sont aussi très critiques quant à leur faible performance. Deuxièmement, ils souhaitent voir des priorités définies alors qu'ils n'ont pas le pouvoir d'y accorder leur financement. Ce ne sont pas eux qui dessinent la politique de leur Etat, ils dépendent d'agenda qu'ils ne contrôlent pas. Ceci explique en partie le faible taux de réponse à l'Appel et aux priorités et l'inadaptation des systèmes de financements aux besoins du terrain. Enfin, ils soulignent leur volonté de faire du développement mais ne disposent pas de fonds à long terme.

\subsection{Humanitaire et politique}

Une des critiques essentielles des ONG et agences des Nations Unies est que le processus favorise la politisation de l'aide.

Les acteurs de l'aide prétendent agir hors de tout agenda politique. Or, depuis le début du conflit une des caractéristiques principales de l'aide en Afghanistan a été son caractère politique. Cependant, dans les années 80 , les intérêts des acteurs humanitaires et des bailleurs de fonds convergeaient dans le soutien aux moudjahidins. La manne des financements déversée sur l'Afghanistan avec un très faible contrôle, permettait aux ONG comme aux agences des Nations Unies d'opérer avec une très grande latitude, et sous couvert d'impératif humanitaire d'apporter un soutien clairement politique en travaillant auprès des populations. Les acteurs de l'aide humanitaire prenaient partie dans le conflit et plus encore étaient utilisés par les états bailleurs de fonds comme « des instruments de leur politique ${ }^{32}$ Etudiant plus particulièrement le cas de l'aide américaine, Helga Baitenmann montre que «l'implication du gouvernement américain ne se limitait pas au financement des ONG, elle consistait aussi à les conseiller quant à leurs programmes. Elles étaient sous haute surveillance; ainsi de nombreuses ONG ont coordonné leurs efforts avec la politique américaine ${ }^{33}$. Ce passé marque aujourd'hui encore directement le paysage humanitaire en Afghanistan notamment à travers les nombreuses ONG toujours présentes qui ont été créées pendant la guerre sur une base idéologique. Leur champ d'action est limité à l'Afghanistan et elles ont parfois gardé des relations privilégiées avec leur gouvernement national ${ }^{34}$.

Suite à la fin du conflit et de la guerre froide, l'Afghanistan n'est plus un enjeu géopolitique fondamental et la diplomatie humanitaire telle que la définit $M$. JC. Rufin : «perd tout à coup son intérêt en même temps que (les conflits) cessent d'être au cœur de l'affrontement stratégiques mondial ${ }^{35}$.

\footnotetext{
${ }^{32} \mathrm{H}$. Baitenmann « Ngos and the politicisation of humanitarian aid », Thirld world quaterly Vol $12, \mathrm{n}^{\circ} 1$ janvier 1990

${ }^{33}$ ibid

${ }^{34}$ On trouve notamment dans cette catégorie des ONG telles que Dacaar (ONG danoise), Swedish Committee for Afghanistan

${ }_{35} \mathrm{JC}$ Rufin « humanitaire et politique depuis la chute du mur de Berlin »in Le piège humanitaire, ed Pluriel, 1994 p353
} 
Cette évolution a laissé de nombreux acteurs de l'aide penser qu'ils allaient pouvoir intervenir en Afghanistan hors de toute considération politique. Ces prétentions à la neutralité sont toujours prégnantes au sein de la communauté de l'aide tant de la part des bailleurs de fonds que des ONG. Les premiers arguent du fait qu'ils n'ont aucun intérêt géopolitique dans le pays et clament que le seul motif de leur intervention est l'assistance à la population afghane. Quant aux $\mathrm{ONG}$, elles s'affirment en tant qu'organismes non gouvernementaux et financièrement indépendants (on peut se demander à l'égard de qui lorsque l'on constate que la majeure partie d'entre elles travaillent sur fonds publiques.) En outre, lorsque l'on interroge les ONG sur les motifs qui les ont poussé à s'impliquer dans la programmation commune, ils citent fréquemment la pression de leurs donateurs.

Ceci étant il serait partial de ne pas ajouter que la multiplicité des donateurs et la variété de leurs agendas laissent une assez grande marge de manœuvre aux organismes d'aide dans la conception de leur politique et de leurs programmes. Il est toujours possible de s'insérer dans la politique de l'un ou l'autre financeur. De plus, rares sont les ingérences des bailleurs de fonds, jusqu'au milieu des années 95 , au niveau opérationnel. Leur principale exigence étant la volonté d'accentuer, au sortir de la guerre soviéto-afghane, les financements en vue des programmes de développement. Ceci n'a pas dérangé les acteurs présents sur le terrain qui se sont adaptés à cette évolution.

En revanche, l'arrivée au pouvoir du mouvement Taliban a bouleversé la donne humanitaire dans la région. La médiatisation simpliste du mouvement place l'Afghanistan dans une position particulière. S'il n'est plus un enjeu géostratégique direct pour la communauté internationale, le pays dérange, il bafoue les normes internationales des droits de l'homme, il réveille les peurs de l'obscurantisme religieux. On ne peut aujourd'hui comprendre l'évolution de l'aide en Afghanistan si on occulte cette dimension de la politique internationale. Les discours relatifs à la montée des extrémismes, notamment religieux font recette. Si la division Est/Ouest du monde a été abolie, de nouvelles lignes de ruptures se dessinent pour certains. Ainsi Huntington affirme-t-il que "le renouveau et l'affirmation culturelle grandissante des sociétés non occidentales et le rejet accru de la culture occidentale iront de pair. Le renouveau des religions non occidentales (l'islam) est la manifestation la plus puissante de l'antioccidentalisme $»$. Or, ces allégations et la peur, voire la diabolisation de l'islam, qui en découlent font recette parmi certains décideurs de la politique étrangère américaine comme dans la presse publique que ce soit aux Etats Unis ou en Europe.

L'opinion publique occidentale nourrie d'une vision simpliste ${ }^{36}$ du conflit afghan et du mouvement Taliban réclame des comptes à ses politiques. Or, les Etats individuellement ni collectivement n'ont pas la volonté politique d'intervenir directement dans la crise afghane, crise confuse où sont trop impliqués les Etats voisins pour qu'une intervention extérieure soit sans danger et emporte l'unanimité. En revanche, si l'Afghanistan est trop loin de nous pour que nous nous en préoccupions directement, il vient aux Etats occidentaux par le biais de mouvements réels. Il est, selon la qualité des récoltes, parmi les trois premiers producteurs mondiaux d'opium; il comporte une forte population réfugiée dans les pays voisins dont beaucoup envisagent une immigration plus lointaine plutôt qu'un retour dans leur pays. La crainte d'un afflux de réfugiés motive largement l'assistance. L'Afghanistan dérange aussi par ce qu'il

\footnotetext{
${ }^{36}$ Courrier international $n^{\circ} 461,2$ septembre 1999, présente Massoud comme le dernier héros luttant contre l'intégrisme
} 
symbolise : la montée du fondamentalisme islamique, les violations des droits de l'homme, (que les états ne peuvent tolérer de la part d'un état qui ne représente aucun intérêt économique). Dans cette région instable, la crainte de la contagion du mouvement fondamentaliste est un élément important. Les Etats ne. peuvent se compromettre à traiter directement avec les Taliban et les tentatives d'enrayer le conflit avec l'UNSMA (Mission spéciale pour l'Afghanistan) se sont soldées par des échecs successifs. La non reconnaissance de l'autorité Taliban fait des acteurs de l'aide internationale les principaux interlocuteurs des Taliban. Comme le souligne M. JC. Rufin les Etats «cherchent avec succès à se soustraire à leurs responsabilités qui sont la recherche de la paix et le développement ${ }^{37}$. La gestion de ces deux thématiques majeures est quasiment abandonnée aux mains des Nations Unies (principalement du volet humanitaire) et des ONG. Dès Ashgabat, les Etats demandent une plus grande collaboration entre les versants assistance et politique de l'activité des Nations Unies, collaboration censée favoriser la construction de la paix. Or, deux ans après le forum, les progrès dans ce domaine sont limités à la présence de représentant de l'UNSMA aux réunions de coordination.

Ces éléments combinés, en dépit des affirmations sur la perte de l'intérêt géopolitique de l'Afghanistan, placent les acteurs de l'aide internationale sous haute surveillance politique et devient de fait un instrument principal de la diplomatie. Ainsi, des enjeux tels que la culture de l'opium, les droits de la femme sont remis entre leurs mains, parce que les Etats n'ont pas les moyens de les traiter à un autre niveau. La communauté d'aide devient un acteur quasi exclusif des relations avec le mouvement Taliban.

Ceci explique l'intérêt des donateurs pour une meilleure coordination de l'assistance. Il ne s'agit pas ici de remettre en cause le dévouement des représentants des bailleurs de fonds sur le terrain et d'en faire de cyniques promoteurs de politique, plus soucieux de la puissance étatique que de l'assistance à la population afghane. Les personnes rencontrées ont au contraire affirmé leur souci constant d'améliorer l'efficacité de la distribution de l'aide afin de mieux servir les bénéficiaires. Mais, ils travaillent dans un contexte qui les dépassent et ne sont que les fonctionnaires exécutants de politique qu'ils ont bien du mal à influencer. S'ils ont une meilleure connaissance du terrain que leur capitale leur voix n'est pas toujours écoutée. Ainsi, lors d'une réunion de présentation de la politique britannique aux $O N G$, la représentante de DFID (Department For International Development) à Islamabad demande de l'aider à plaider contre la mise en œuvre de sanctions contre l'Afghanistan et de lui fournir des arguments contre l'interdiction d'envoyer des expatriés citoyens du Royaume Uni dans le pays, afin de convaincre son gouvernement de modifier sa position.

\subsubsection{Vers une politisation accrue de l'aide?}

La promotion d'une meilleure coordination présente un double avantage pour les Etats. En premier lieu, elle permet une meilleure lisibilité du paysage de l'aide. Le risque comme le déclarent des responsables de Médecins sans Frontières est de voir «imposé à l'ensemble des ONG une approche humanitaire centralisée unique et intégrée ${ }^{38}$. Alors que l'on sait combien la variété des problématiques nécessite une capacité d'adaptation et une connaissance du milieu de l'intervention que les ONG plus proches des populations sont mieux à même de développer que les Nations Unies. Elles jouissent d'une plus grande liberté d'action, d'une plus grande présence

\footnotetext{
37 ibid. p354

${ }^{38}$ P.Salignon, P-P. Vandini " quelle coordination? », Les Nouvelles d'Afghanistan, $n^{\circ} 85,27^{\text {ème }}$ trimestre 99, p5
} 
sur le terrain et donc d'une meilleure capacité d'analyse. C'est leur créativité et la pluralité des approches qui fait leur richesse.

En second lieu, le leadership onusien assure aux Etats une meilleure prise en compte de leurs agendas politiques que le canal des ONG par trop indépendantes et moins facilement contrôlables. Nous ne pouvons, à la lumière de l'analyse de l'assistance à l'Afghanistan, que souscrire à l'affirmation de M.JC. Rufin: "Les Nations Unies sont aujourd'hui une pièce prépondérante de l'humanitaire d'état; la principale vertu de l'organisation est de conférer sur le terrain un degré supérieur de légitimité et une apparence de neutralité aux interventions étatiques. $1{ }^{39}$

Le processus de coordination, par la place qu'il réserve aux donateurs, leur offre une occasion sans précédent de s'ingérer dans l'élaboration des politiques d'assistance des organismes humanitaires et de développement et d'imposer leur vision. En effet, la mise en place de mécanismes, permettant une planification commune des stratégies et des priorités par l'ensemble des acteurs, donne à celui qui détient le pouvoir financier et en dernier ressort le pouvoir de décision, une voix naturellement plus importante que celles des autres parties et ce, quelles que soient les garanties offertes en terme de parité de représentation. Dans un contexte de compétition exacerbée pour les fonds, la tendance est de chercher à se conformer aux volontés supposées ou connues des bailleurs de fonds plutôt que de répondre aux besoins analysés sur le terrain. C'est ainsi que des thèmes tels que la drogue, les droits de la femme, le soutien aux communautés sont placés en priorité sur l'agenda des acteurs de l'aide alors qu'ils ne correspondent pas forcément aux priorités sur le terrain.

Le programme commun du Hazarajat pour l'Appel Consolidé 2000 donne une autre illustration de ce type de déviance. Il propose, pour répondre au déficit alimentaire, de mettre en place des programmes denrées alimentaires ${ }^{40}$ contre travail (food for work) financés par le PAM. Comme l'explique le coordinateur régional, cette décision n'est pas fondée «sur une réflexion sur la meilleure politique à mettre en œuvre mais sur la connaissance des disponibilités en blé du PAM ${ }^{41}$. Les effets pervers de ce type de programme qui ne couvre qu'une partie des besoins alimentaires de la population ne sont nullement pris en compte.

\subsubsection{La sécurité comme prétexte}

Mais ce n'est pas dans ce domaine que les dangers de politisation ont été exprimés par les ONG. Leurs réactions ont été déclenchées par les restrictions à l'envoi sur le terrain d'expatriés, imposées par la Grande Bretagne et les Etats Unis ${ }^{42}$ et par la déclaration collective des donateurs lors de la réunion du groupe de soutien à l'Afghanistan demandant aux ONG de « soutenir la position des Nations Unies en matière de sécurité, plus particulièrement en ne renvoyant pas d'expatriés en Afghanistan ${ }^{43}$. Les ONG protestent contre cette sous-estimation de leur capacité à traiter des problèmes de sécurité. Elles estiment que cette restriction n'est autre qu'une tentative de politisation de l'aide, une manière déguisée d'imposer une conditionnalité de

\footnotetext{
${ }^{39}$ Rufin p354

${ }^{40}$ les denrées fournies sont du blé et de la farine

${ }^{41}$ entretien avec M Semple

${ }^{42} \mathrm{La}$ Grande Bretagne ne finance plus les ONG qui ont des expatriés permanents en Afghanistan

${ }^{43}$ Chairperson summary of the fourth meeting of the Afghanistan Support Group, Nobutaka Machibura, Tokyo, 1999
} 
l'aide. ${ }^{44}$ Si les bailleurs de fonds n'ont pas tous adopter ces restrictions extrêmes, la volonté d'obtenir un consensus les amènent à soutenir la position onusienne.

\subsubsection{Des principes « en principe » à la conditionnalité de l'aide}

L'effort de coordination avait, dès les débuts de l'initiative, pour objectif d'apporter une réponse unifiée de la communauté internationale aux violations des droits de l'homme en Afghanistan sans abandonner les populations bénéficiaires. L'accent étant mis sur les principes sur lesquels devaient reposer toute action humanitaire et de développement. Ce thème a fait l'objet de nombreuses publications révélatrices de la difficulté de parvenir à un consensus. Ainsi l'incohérence est frappante lorsque l'on constate qu'aucune des versions de la stratégie d'assistance ni du «Making a reality of Common Programming »'en donnent une liste identique.

La question des principes est récente en Afghanistan. En effet, dans les années 80, les principes d'impartialité et neutralité n'étaient pas de mise. Le pillage de l'aide n'avait donné lieu à aucune investigation, et chacun s'en accommodait plus ou moins de manière pragmatique sans que des approches communes ne soient discutées tant au sein des Nations Unies que des ONG $\mathrm{La}$ «question des femmes» n'en était pas une, et les multiples violations des droits de l'homme, pas plus que le détournement de l'aide et le racket des organisations n'ont fait l'objet de débats internes à la communauté d'aide ou aux donateurs, ni de débats publiques médiatisés en Occident. Les autorités Taleban n'ont d'ailleurs pas manqué de faire remarquer à leurs interlocuteurs qui opposaient des arguments de principe que ces préoccupations semblaient bien soudaines.

Si le cadre stratégique pose en principe que l'aide au développement et les projets de réhabilitation ne doivent être apportés que dans la mesure où il ne renforce pas les parties en guerre de manière politique ou militaire. C'est certainement la politique des Taliban à l'égard des femmes qui a été le catalyseur d'une telle émotion au sein de la communauté internationale, non pas que celle-ci soit dans le fond pire que les pratiques sous les régimes précédents, mais le caractère officiel des discriminations est apparu choquant et insupportable. De plus, la médiatisation de cette question a forcé la main aux gouvernements qui se sont vus dans l'obligation de montrer à leur opinion publique qu'ils ne restaient pas indifférents à la situation des femmes afghanes.

La question des principes et de leur application est loin d'être résolue et si le processus a eu le mérite de poser le débat, il ne semble pas à ce jour qu'un consensus ait été atteint. Tout le monde s'accorde aujourd'hui pour dire que l'aide doit reposer sur des principes, mais la question de savoir lesquels et comment les mettre en pratique reste entière. "Les principes oui mais... ». En ce qui concerne les relations avec les autorités, il semble faire unanimité qu'elles ne doivent pas être impliquées à haut niveau dans les structures du PCP, ainsi personne n'envisage qu'elles soient membres des organes nationaux. Lors d'une récente réunion à Peshawar, l'ensemble des participants (ONG et agences des Nations Unies) se sont mis d'accord pour qu'elles ne soient pas non plus membres des organes régionaux de coordination, en revanche elles sont invitées aux meetings de coordination dans le Hazarajat. En général, on tente de s'entendre avec les autorités locales et techniques en évitant de renforcer les autorités centrales et politiques. Cependant, dans certains cas, des agences des Nations Unies travaillent en collaboration avec les ministères. C'est

44 " Politicization of Aid for Afghanistan » Statement by the APB NGO member, february 99 
le cas de l'UNICEF qui travaille avec le Ministère de la Santé (MOPH) sur ses programmes de vaccination.

Ainsi, il semble que l'on se trouve toujours dans la situation classique où chacun détermine en fonction de son mandat et de ses besoins la manière d'interpréter et d'appliquer les principes. Est-ce à dire qu'aucun consensus n'est envisageable en la matière ? Que l'emphase mise sur les principes n'est qu'hypocrisie? La communauté internationale est beaucoup moins regardante sur les droits de l' homme lorsque des intérêts commerciaux sont en jeu, doit-on pour autant renoncer à ces principes? Si l'Afghanistan doit servir de pays modèle c' est peut être la première question qui devrait être soulevée ailleurs et pas uniquement en ce qui concerne l'aide internationale !

Une approche commune est cependant nécessaire afin de sortir de l'impasse qui mène à une conditionnalité de l'aide. En effet, l'approche actuelle tend à confondre l'impératif d'assistance à la population en fonction de principes humanitaires de non-discrimination qui implique une réflexion sur la meilleure façon de venir en aide aux bénéficiaires avec l'imposition de conditions aux autorités leur demandant de mettre elles-mêmes en œuvre ces principes. Il convient, plutôt que de théoriser sans fin sur le soutien que constitue ou non l'aide au mouvement Taliban, de chercher les exemples pratiques qui permettent de répondre aux violations des droits de l'homme.

\subsubsection{Le PCP, un facteur de politisation?}

Le processus ne représente pas en soi un facteur de politisation. Les bailleurs de fonds, ont, nous l'avons vu avec la fermeture des programmes à Kaboul en 1998, les moyens d'imposer des conditions à leur assistance quelque soit le degré de coordination. En revanche, le risque existe si la coordination au lieu de renforcer le dialogue tend à donner tout le poids au pouvoir financier. Les $O N G$ doivent cesser de se parer de leur neutralité pour ne pas prendre leur responsabilité, elles doivent davantage s'organiser pour peser dans les débats, l'acte d'assistance s'il est neutre au regard du pays dans lequel il intervient est en soi un acte politique de « refus de l'injustice ». Elles doivent se donner les moyens d'agir; ceci passe par un renforcement de leur propre cohésion et par une mobilisation des sièges qui doivent à leur niveau mobiliser les opinions publiques. Si elles n'ont pas le courage de prendre cette responsabilité, il est à craindre que la politisation de l'aide ne se fasse aux détriments des intérêts des populations concernées.

Pourquoi ne pas envisager d'imposer des conditions aux bailleurs de fonds et de refuser collectivement les financements de ceux qui tendent à politiser à leur profit l'action des ONG. Elles trouveront sans doute un soutien auprès de donateurs soucieux d'apporter une véritable assistance aux populations. 


\section{TROISIEME PARTIE : \\ PCP ENTRE RHETORIQUE ET REALITE}

Si le PCP a fait couler beaucoup d'encre et soulevé de nombreux débats aucune évaluation de sa portée réelle, de l'ampleur des changements effectués ou non sur le terrain n'a à ce jour été conduite. Cette demande est pourtant récurrente de la part des différents acteurs. Il ne s'agit pas ici de répondre de façon non exhaustive à la question de savoir si le processus a été ou non à l'origine d'une meilleure coordination de l'assistance en Afghanistan et au-delà d'une plus grande habilité à répondre aux besoins de la population afghane. Ce travail dépasse largement nos compétences et moyens de travail. En revanche, nous allons tenter de dresser un tableau de la situation à ce jour à travers l'exemple d'une coordination régionale, et de voir de quelle manière les acteurs tentent de mettre en œuvre ou non une nouvelle approche, dans quelle mesure celle-ci a des conséquences sur la nature de l'aide apportée.

\section{LES MODALITES DE LA CONSULTATION}

Avant d'étudier plus précisément les résultats du processus, il est impératif d'examiner la manière dont la consultation des différents acteurs a été menée, celle-ci aura en effet des répercussions sur les succès et échecs du processus. Les premiers malentendus sont perceptibles dès Ashgabat. La volonté affichée par les Nations Unies de prendre via UNOCHA, le leadership de l'aide a été très mal perçue par les ONG. L'histoire des relations entre les agences des Nations Unies et les ONG est faite de mépris réciproque et de mésentente. Les secondes accusant les premiers d'être inefficaces, de ne pas connaître les réalités du terrain et de les considérer avec arrogance et non pas en véritable partenaire. Si on ajoute à cela une certaine méconnaissance du système onusien et la confusion volontaire ou non entre les rôles respectifs des agences et de l'UNOCHA, les critiques vont à l'encontre du système onusien dans sa globalité. La réaction est donc quasi épidermique: "nous ne voulons pas être dirigés par les Nations Unies», le commentaire d'un volontaire d'une ONG française ; «les Nations Unies veulent avoir la mainmise sur l'ensemble de l'aide apportée à l'Afghanistan » est largement partagé. On met en relief l'arrogance d'une institution réputée pour sa piètre efficacité et qui prétend apprendre aux autres à mieux travailler et se coordonner.

Cependant les ONG sont aussi conscientes de la pression des donateurs et du caractère imparfait des mécanismes en place. L'idée d'améliorer les performances de la communauté internationale peut difficilement être attaquée en soi, quiconque se tient à l'écart du processus prend le risque d'être taxé de sabotage. A l'exception de quelques grosses ONG internationales telles que MSF, les ONG décident donc au travers de ACBAR et des autres coordinations, de prendre part au processus.

\section{- Des espaces de dialogue réduits}

Il semble en outre que l'implication des ONG, si elle est part de tous les discours n'ait pas été une réelle priorité pour les personnes chargées de la mise en œuvre du PCP. Ces litiges sur la forme de la consultation sont lancinants tout au long du processus. Les différents groupes de travail et réunions, ont souvent lieu à Islamabad, les ONG prévenues qu'au dernier instant n'ont pas toujours le temps nécessaire pour s'y rendre ou y envoyer du personnel qualifié, les 
critiques quant à la somme de documentation envoyée en dernière minute en préparation des réunions sont elles aussi récurrentes, «c'est comme si tout était fait pour que nous ne lisions pas les documents et que nous ne puissions pas nous déplacer à temps ».

Ce n'est pas seulement la forme de la consultation qui pose question quant à un réel désir d'impliquer les $\mathrm{ONG}$, mais surtout le fait que leurs remarques ne soient pas prises en compte. Depuis le début de processus, elles demandent que des problèmes concrets tels que l'harmonisation des salaires des agences des Nations Unies et des ONG soient réglés mais rien n'est fait dans ce sens. L'idée de financement commun si elle n'est plus un objectif à court terme apparaît toujours en arrière plan du "Making a reality » et nous verrons qu'elle revient subrepticement à l'ordre du jour dans certaines régions. Le caractère factice de la consultation apparaît plus clairement dans la prise en compte des réactions au texte « Making a reality », des critiques parfois très pertinentes n'ont jamais trouvé d'écho. Les promoteurs de la coordination sont davantage des professionnels de la communication qui manient l'art de la persuasion beaucoup plus aisément que celui de l'écoute

En dépit des velléités d'indépendance des agences des Nations Unies et afin de gagner leur soutien, une large place leur est réservée au sein des organes imaginés. Ainsi, elles sont membres de droit des groupes régionaux, à la tête des groupes techniques et toutes les agences ont droit à une place au sein de l'instance nationale de coordination. Cette question des modalités de participation des différents groupes d'acteurs sera l'objet de multiples débats en 1998, les ONG adoptant une position d'attaque systématique, se sentant largement infériorisées au sein des organes. La question est en effet cruciale pour les Nations Unies qui doivent prouver aux donateurs qu'ils sont effectivement les dirigeants de la coordination en Afghanistan. Le débat sur la direction de la coordination prend donc le pas sur celui de son efficacité, l'essentiel étant de montrer qu'il existe des structures qui se réunissent, peu importe la qualité de celles-ci. La réalité des débats n'est pas toujours reflétées dans les minutes des réunions qui soulignent toujours le caractère consensuel des décisions et mettent en avant des conclusions qui n'ont pas forcément été prises au cours des réunions. Le nombre de participants est considéré comme un facteur de succès, alors que l'absence de personnel qualifié ou ayant un pouvoir décisionnel au sein de leur organisme est fréquente. Ceci remet en cause l'utilité même des débats. "Certains envoient aux réunions, quiconque n'avait rien à faire ce jour là, d'autres ne s'y rendent que parce que le repas est fourni mais n'ont rien à apporter aux débats ». Ce sont les fondements de la coordination choisie qui sont alors questionnables. En effet, elle repose essentiellement sur des réunions formelles et fréquentes, or ceci représente une charge de travail supplémentaire conséquente notamment pour les ONG qui n'ont pas toujours les ressources humaines suffisantes.

\section{LES STRUCTURES MISES EN PLACE}

\subsection{Au niveau national}

\subsubsection{A.P.B.: Organe de Programmation pour l'Afghanistan}

C'est la structure nationale de coordination, elle se réunit une fois pas trimestre. Le rôle de l'APB consiste à :

- faire des recommandations de principe sur les questions concernant le PCP

- soutenir le mécanisme du C.A.

- promouvoir une coordination effective 
Il est composé des trois groupes représentés de façon paritaire, chacun choisissant ses représentants. L'APB devait être doté d'un secrétariat dont le rôle consiste à transformer les recommandations en pratiques et à assurer une bonne communication entre le terrain et la base. Il s'agissait, prévoyant les difficultés de l'APB à être opérationnel, de le doter d'un organe plus pratique. Mais en l'absence de moyens suffisants, le secrétariat de l'APB est assuré par le Coordinateur des Nations Unies. Ceci pose une question quant à l'indépendance du secrétariat à l'égard des Nations Unies, le but de l'APB étant de développer le consensus. Ces réunions représentent une occasion pour les 3 parties de l'aide de se rencontrer et de se connaître. Ce d'autant plus que des sous-groupes de préparation se forment. Ainsi lors de la réunion de juin, ONG et Nations Unies ont présenté des revendications communes.

Ces réunions sont très formelles et permettent la présentation des enjeux tels que la politisation de l'aide ou des problèmes de sécurité. Chacun peut ainsi prendre connaissance de la position des autres. En revanche, le caractère opérationnel de l'APB est limité voire inexistant. En effet, les décisions ne peuvent être prises qu'à l'unanimité. Les agences des Nations Unies et ONG s'accordent à dire que la valeur ajoutée de l'APB à l'aide internationale est nulle. Une des principales raisons réside dans sa constitution. En effet, en dépit de son peu d'efficacité, l'APB revêt un caractère tel que personne ne veut en être écarté, ses réunions rassemblent ainsi plus d'une quarantaine de personnes auxquelles s'ajoutent parfois autant d'observateurs. Ceci entraîne une incapacité quasi génétique à prendre des décisions et à remplir son rôle premier qui est de bâtir un consensus sur les problèmes concernant la communauté d'aide internationale dans son ensemble.

Le constat de cet échec étant partagé son président actuel, Victor Carvell, représentant canadien, tente de le réformer afin de le rendre plus opérationnel. Il a fait le choix d'organiser des réunions professionnelles sur des thèmes précis, ce qui permet d'éviter de voir les réunions encombrées par des personnes qui ne connaissent pas les problèmes. Cependant, la question de savoir si cela permettra ou non d'établir un véritable échange entre des participants aux intérêts divergents reste entière.

\subsubsection{ASG : Groupe de soutien à l'Afghanistan}

Celui-ci est censé assurer un meilleur dialogue entre les acteurs de l'aide et les donateurs. Il présente les mêmes défauts que l'A.P.B., à savoir un caractère formel, un trop grand nombre de participants. Il est davantage une tribune où chacun présente ses revendications qu'un lieu de concertation. La présence de fonctionnaires des administrations centrales des pays membres en fait un forum plus politique que tourné vers l'assistance.

\subsubsection{Les groupes thématiques ${ }^{45}$}

Ils seront présentés dans le cadre de l'Appel Consolidé

\subsection{Au niveau régional}

Le texte "Making a reality » prévoyait la mise en place d'organes de coordination régionale dans les 7 régions définies par l'UNOCHA. La division géographique de l'Afghanistan en ces 7 régions ne reflète pas les divisions géographiques ni économiques du pays. En dépit des critiques envers ce découpage arbitraire, aucune modification n'a depuis été apportée, il ne

${ }^{45}$ voir annexe $n^{\circ} 8$ 
permet en rien de développer des stratégies de développement régional puisqu'il rassemble des districts qui n'ont pas de problèmes communs et parfois pas de voies de communication. ${ }^{46}$

Afin de faciliter et d'animer ces organes, UNOCHA a recruté en 1998 cinq coordinateurs régionaux (RCO). Lors de l'été 98 des organes régionaux $(\mathrm{RCB})$ ont donc été constitués dans quatre régions, après maintes discussions sur leur composition et leur rôle. Leur rôle est de permettre une meilleure prise en comte des besoins évalués dans la programmation commune. Ce sont les canaux d'un partage de l'information, qui leur permettent de déterminer les priorités régionales. Ils sont assistés dans cette tâche par des groupes de coordination sectorielle, chargés de l'harmonisation des approches techniques : les « groupes de coordination ». Leur nombre et thèmes varient selon les régions. Dans certaines régions (Est), ils étaient préexistants aux processus de coordination. Ils recoupent aussi certains groupes mis en place par ACBAR. Leur efficacité est plus encore que, dans les autres groupes puisqu'il s'agit de problèmes techniques, fonction de la compétence des personnes qui y participent. Leur fonctionnement montre des résultats variés, qui reposent en outre sur la motivation des personnes impliquées. Cependant, les rares groupes qui fonctionnent ne sont en rien liés au reste de l'architecture.

Mais l'expulsion des ONG de Kaboul puis l'évacuation du personnel international onusien et des ONG suite aux bombardements américains ont paralysés les organes régionaux et on peut aujourd'hui affirmer qu'aucun d'entre eux n'a assumé sa tâche. A ce jour, 4 coordinateurs régionaux ont été recrutés, seuls celui de Kaboul et de Kandahar sont basés en permanence en Afghanistan.

\subsubsection{Kaboul}

L'étude de la coordination à Kaboul dépasse le cadre de ce travail, puisque nous n'avons pas eu les moyens d'y séjourner. Il convient cependant de préciser qu'elle est rendue beaucoup plus aisée par le peu d'acteurs engagés. En effet, depuis l'évacuation de 1998, nombreuses ONG n'ont pas repris ou ont fortement réduit leurs programmes. Il semble que les ONG présentes soient satisfaites des mécanismes mis en œuvre, l'accent est mis sur la coordination sectorielle des activités. La personnalité du coordinateur régional est un facteur important de cette réussite, doté d'une longue expérience de l'Afghanistan, il jouit d'un prestige suffisant pour être écouté par les acteurs, y compris les agences onusiennes. Cependant, sa tâche est complexifiée, car situé dans la capitale, il joue un rôle de représentation auprès des autorités. Il prend actuellement en charge un processus de dialogue avec les Taliban prenant pour base le mémorandum signé en 1998.

\subsubsection{La région nord}

La région nord a cette particularité de se trouver dans les zones de combats actifs, très peu d'ONG y sont engagées et ce essentiellement dans des actions d'urgence. Ce sont en outre des ONG traditionnellement opposées à la coordination de type MSF. Les agences des Nations Unies y sont très peu nombreuses et les questions de sécurité empêchent d'avoir une présence expatriée forte sur le terrain. Le RCO, basé à Islamabad, est le centre d'une coordination qui ne dépasse pas la coordination opérationnelle classique, il n'a les moyens ni d'imposer une réflexion commune sur les problématiques de la région ni de combler les lacunes dans la couverture géographique de l'aide. Ainsi, il n'a pas pu répondre au fort déficit du Badakhshan en terme de présence d'organismes d'aide.

${ }^{46} \mathrm{cf}$ annexe $\mathrm{n}^{\circ} 3$ 
La principale activité a consisté pour l'UNOCHA durant l'été 99 en la coordination de l'assistance aux déplacés de la vallée de Shomali. Un appel a été lancé plus de deux mois après les événements, un groupe de travail a été créé à Islamabad en vue d'élaborer une réponse commune, un appel commun sous l'égide de l'UNOCHA a été lancé. Les fonds sont redistribués par l'UNOCHA aux ONG, il exerce un contrôle en effectuant ses versements par tranche de 30.000 dollars. Cependant, certains donateurs ont exprimé leur mécontentement face à la lenteur du processus, la difficulté d'obtenir des statistiques unifiées, la compétition entre agence, l'UNOCHA censé servir de garde fou, par un suivi rapproché des ONG, n'a pas fait preuve d'une efficacité, les ONG sélectionnées par lui n'étaient pas toujours compétentes.

Selon le coordinateur régional, les structures proposées ne sont en aucun cas adaptées à la situation qui prévaut dans le nord. En outre, les Nations Unies sont très mal placées pour intervenir dans ce type de contexte en raison des trop strictes régulations de sécurité, qui limitent la souplesse d'intervention et la capacité à connâ̂tre réellement le terrain. « Ce n'est pas étonnant lorsque l'on sait que les personnes qui ont dessiné les schémas n'avaient aucune connaissance des réalités afghanes $»$, précise-t-il.

\subsubsection{Le Hazarajat : une coordination imposée}

Nous étudierons les structures mises en place à travers l'exemple du Hazarajat. Le choix de porter plus particulièrement notre attention sur cette ère est du, hormis le fait que MADERA y travaille, à ses caractéristiques. L'intérêt des acteurs de l'aide pour le Hazarajat est récent, c'est seulement suite aux événements de 98 qu'elle s'est retrouvée sous les feux de la rampe. La tentative de coordination mise en œuvre depuis 1997 n'a donc trouvé aucune base sur lesquelles s'appuyer. C'est un exemple typique de coordination par le haut.

\section{- Une situation d'urgence " chronique "}

Dans le cours des années 1997 et 1998 à la suite de deux récoltes catastrophiques, le Hazarajat vit ses moyens d'autosubsistance très fortement diminués, aggravant dramatiquement les problèmes d'approvisionnement résultant d'une guerre civile particulièrement âpre sur les confins de la région, des programmes d'aide alimentaire d'urgence furent mis en place. Malgré cela, en bien des endroits reculés, où cette aide ne pu être acheminée faute de routes et de pistes, de nombreux décès furent à déplorer. Cependant, bien que les bouleversements politiques favorisent l'installation des marasmes alimentaires, ces catastrophes n'ont pas toujours forcément eu, dans l'histoire, un conflit pour origine. C'est que cet Afghanistan central, plus que toute autre région, accumule les handicaps climatiques, géographiques, démographiques, politiques, culturels, historiques et administratifs. Comme le souligne Alain, directeur technique à MADERA : « Ce serait avoir une bien courte vue des choses que de circonscrire les difficultés actuelles du Hazarajat qu'au seul domaine des affrontements politico-militaires. Il y a bien d'autres éléments à prendre en considération si l'on veut pouvoir apporter des réponses plus durables que celles des simples distributions de rations alimentaires. » Cependant c'est sur une thématique de réponse à une crise alimentaire d'urgence que la coordination a été organisée au Hazarajat.

La région a été l'objet depuis deux ans d'attention particulière, reliée en 98 à la région nord, elle dépend cette année de Kaboul mais un coordinateur propre à cette région a été nommé en mars. Le Hazarajat est considéré par certains comme un modèle de mise en œuvre réussie du Common Programming. En effet, les actions qui y ont menées ont fait l'objet d'une grande publicité. Elle a en outre l'avantage d'être une région qui a du sens, et présente une certaine unité. Les Nations Unies refusent d'adopter officiellement le terme courant pour la région : le 
Hazarajat (du nom d'un groupe ethnique majoritaire) car elle estime cette décision trop politique, ils utilisent officiellement le terme de région centre.

Elle avait été en juin 1998 choisie comme région d'essai pour la mise en cuvre du Common Programming. En juillet, était créé un organe de coordination régionale lors d'une réunion a Bamyan (capitale du Hazarajat). Celle-ci reste dans les mémoires de ceux qui y ont assisté comme un modèle de la méconnaissance du personnel des Nations Unies des réalités du terrain et de l'absence de dialogue avec les ONG. Cette tentative de mettre en place un programme commun régional, "central highlands common programm », a avorté en raison de l'évacuation du personnel international.. Le choix de cette région était insensé pour quiconque connaissait un temps soit peu la situation politique, et l'initiative a donc été tuée dans l'œuf, alors que les réunions de juin et juillet avaient été organisées à grand renfort de propagande. Un mois plus tard, il ne restait plus rien des efforts entrepris.

\section{- 1999 : la coordination sur la sécurité alimentaire}

En mars 1999, un nouveau coordinateur régional est nommé. Il commence par une coordination qui se veut opérationnelle sur le thème de la sécurité alimentaire. En effet, la région fait face à un fort déficit alimentaire, conséquence cumulée du blocus mis en place par les Taliban en 1997 et levé en août 1998, de la difficulté d'accès et de la succession de mauvaises récoltes. Des programmes de « argent contre travail », "Cash for Work » et de distribution sont notamment mis en œuvre, ils sont financés par ECHO (European community humanitarian office) et le PAM. La coordination est organisé par le coordinateur régional embauché par UNOCHA, elle rassemble les ONG mettant en œuvre les programmes et le PAM. Elle se donne pour but de s'assurer de la bonne adéquation entre les besoins et les programmes essentiellement au niveau géographique. Elle est organisée par le coordinateur régional par le biais de réunions quasi mensuelles organisées sur différents sites de façon rotative. L'évaluation du travail effectué n'a pas encore été réalisée en raison de problèmes de recrutement. Il semble cependant que la coordination ait facilité une bonne répartition géographique du travail entre les ONG, le partage d'information et a permis aux ONG présentes d'apporter une réponse plus rapide aux zones les plus touchées.

Les facteurs qui ont permis le fonctionnement de cette coordination sont liés aux spécificités de l'échiquier de l'assistance dans la région. La présence d'un nombre limité d'acteurs, seuls quelques ONG et trois agences des Nations Unies, est un atout. Le coordinateur régional le déclare «si la coordination fonctionne dans le Hazarajat c'est sans doute parce que les Nations Unies y sont quasiment absentes ». Au-delà, les acteurs ne sont pas en compétition; la région est vaste et loin d'être saturée. Dans une situation d'urgence alimentaire, la définition des priorités ne fait pas l'objet de débats puisque les besoins sont évidents pour tous. La coordination est davantage opérationnelle qu'une tentative de réflexion globale sur la stratégie à mettre en œuvre dans la région.

La personnalité du coordinateur régional est ici encore un atout important. D'une part il connaît bien la région, d'autre part, il a travaillé avec les ONG ce qui facilite le dialogue, comme le font remarquer certaines personnes interrogées : «il ne se comporte pas avec l'arrogance des autres personnes des Nations Unies $»$.

Cependant, au cours de la mise en œuvre des programmes les premiers problèmes apparaissent. Une des ONG de la coordination, OXFAM, est chargée du suivi général du programme ce qui créé certaines tensions. Sa neutralité est remise en cause, certains expriment les craintes que des critiques soient non fondées et visent simplement à évincer les 
autres ONG pour prendre en charge l'exécution des programmes. Les réunions mensuelles de coordination ne permettent pas d'apporter de réponses communes aux problèmes rencontrés, elles sont restreintes à des évaluations quantitatives peu précises de l'équivalent blé déversé sur la région. Ainsi, dans le cadre des programmes de "Cash for Work » plusieurs ONG sont confrontées au travail des enfants mais à aucun moment une solution commune n'est recherchée. Ces difficultés sont évoquées uniquement lors de la réunion finale, mais le temps de réflexion est limité à quelques minutes, aucun partage d'expérience n'est vraiment possible. Il semble que la résolution de ces questions ne soit pas le principal objectif du coordinateur.

\section{- Accélération et dérapage de la coordination}

Le coordinateur a aussi pour mandat de mettre en oeuvre le PCP et de donner les preuves que celui-ci peut fonctionner. Il doit organiser la structure régionale de coordination. Ceci présente un véritable défi, dans cette zone montagneuse et reculée, le manque d'infrastructures de communications tant physiques (routes) que matérielles (seul le téléphone satellite est fonctionnel) rend difficile les contacts entre $\mathrm{ONG}$, nécessaires à toute coordination.

La structure mise en place permet de s'adapter à ces particularités, des groupes de coordination sont organisés au niveau des districts, chacun est dirigé par une ONG bien implantée dans la région. Un organe de coordination régional (RCB) est formé durant l'été. Mais cette initiative est davantage poussée par le coordinateur régional que le fruit d'un dialogue quant à ses objectifs et son rôle. Il semble que ses membres ne soient pas tous conscients de l'existence d'une architecture complexe dans laquelle il s'insère ${ }^{47}$.

La préparation de l'Appel illustre la position difficile dans laquelle se trouve le coordinateur partagé entre un exigence de résultats et sa conscience de la complexité des problématiques dans la région. Deux mois avant la sortie de l'Appel, la décision est prise d'élaborer une fenêtre commune régionale afin de mettre en avant une approche plus pertinente que celle des groupes thématiques. Le coordinateur régional sollicite l'assistance des $\mathrm{ONG}$, favorables à cette approche, pour l'élaboration de cette fenêtre, mais prise dans leurs problèmes opérationnels celles-ci n'ont pas de temps à y consacrer et se contentent d'inscrire de manière individuelle leur projet. Le jour précédent la date butoir, un programme commun rédigé par le seul coordinateur est présenté. Celui-ci sera présenté au nom du RCB, mais écrit à la hâte il ne fixe que les grandes lignes des projets sans donner de détail sur les agences mettant en oeuvre le programme. L'UNOCHA est de fait l'agence qui recevra les fonds accordés et sera chargée de la redistribution. Il s'agit donc de la réapparition du fond commun refusé par l'ensemble des acteurs lors des débuts du processus. Les ONG y sont particulièrement opposées. « Leur indépendance financière vis-à-vis des Nations Unies constitue leur unique moyen de discuter stratégie avec elles. Sans cela, elles seront réduites au rôle d'agence d'exécution sans aucun pouvoir sur la définition des stratégies et objectifs. Or, les Nations Unies n'ont pas jusqu'à présent montré une ingéniosité dans leurs interventions qui justifie un tel monopole ${ }^{48}$.

Cet exemple montre que la coordination ne fonctionne jusqu'ici que dans des situations d'urgence, nous sommes encore loin de la programmation commune. Celle-ci nécessite davantage de dialogue entre les acteurs. C'est un processus long qui ne peut reposer que sur des bases solides et une analyse en profondeur de la région. La pression des donateurs et de la direction de l'UNOCHA leurs attentes ont, dans ce cas, entravé le processus de coordination.

\footnotetext{
${ }^{47}$ A la question posée lors d'un meeting, "voulez-vous ou non former un RCB ? ", la seule réponse fut des hochements de tête aussitôt pris pour un acquiescement.

${ }^{48}$ Arnaud Cauchois, note interne, MADERA, Peshawar, novembre 99
} 
L'impératif de résultat a entraîné le coordinateur régional à agir seul et vite au détriment de la consultation et de la qualité des débats. Les ONG ont, elles aussi, leur part de responsabilités, prises dans la gestion courante de leurs programmes, elles ne s'intéressent pas suffisamment à la coordination. Cette expérience met aussi en exergue, la nécessité de mieux former les personnes qui sont envoyées aux réunions de coordination afin qu'elles puissent défendre le point de vue de leur organisme. Elle souligne encore la difficulté de mener à bien une coordination lorsque celleci est liée à la récolte de fonds. Comment, en effet, prétendre coordonner les acteurs lorsqu'on les situe sur un marché compétitif. Il est impératif de séparer les questions financières et stratégiques. Le fonds commun n'est pas un palliatif puisque les acteurs se trouvent alors en compétition pour sa gestion. Les donateurs doivent assumer leur rôle qui est de choisir la destination de leurs fonds et se donner les moyens de discerner les programmes qui répondent le mieux aux besoins. La tentative d'imposer la coordination par une approche alternant le bâton et la carotte, les menaces de réductions de fonds et les promesses de leur augmentation, n'est pas fructueuse mais contribue à biaiser le processus. Il est en effet facile de faire croire qu'une coordination fonctionne. Mettre en place un réel dialogue requiert un processus long, il convient de donner aux acteurs les moyens financiers et le temps de s'y impliquer.

\subsection{L'Appel Consolidé}

\subsubsection{Définition}

Ce document lancé pour la première fois en 1992, suite à la création du DHA, est à l'origine une compilation des demandes de financement propres aux agences des Nations Unies travaillant dans le domaine de l'urgence. Il s'agit d'une source de financement additionnel aux fonds propres des agences afin de pallier les manques de ressources pour les pays en crise. Suite à la réforme de 1997, le processus de l'Appel fut renforcer par l'inclusion de nouvelles agences, effectuant des programmes de réhabilitation et reconstruction. Dans le cas de l'Afghanistan l'Appel fut aussi élargi aux ONG.

Ce processus, antérieur au PCP, est l'objet de critique quant à son manque de cohérence, simple addition de projets parfois irréalistes de surcroît des agences. Il ne reflète ni la réalité de leur capacité ni une réflexion commune sur les objectifs à atteindre. Pourtant sa définition est beaucoup plus ambitieuse : il s'agit « d'un processus de programmation par lequel les systèmes d'assistance nationaux, régionaux et internationaux (...) sont capables de se mobiliser et de répondre aux urgences complexes ${ }^{49} \gg$.

\subsubsection{L'appel et le PCP}

Dès le début de la concertation sur la coordination les deux processus ont été liés. L'Appel Consolidé étant considéré comme un outil permettant de mesurer les avancées en terme d'élaboration de stratégies communes.

Etant donné les très faibles progrès réalisés en matière de coordination depuis le début du processus de programmation commune, l'Appel est pour beaucoup considéré comme le seul résultat tangible, voire comme une fin en soi, par la publication d'un document qui ne soit pas une simple liste de projets présentée comme une preuve d'une meilleure coordination. L'UNOCHA ne ménage donc pas les efforts en vue de sa rédaction, qui est l'occasion de

${ }^{49}$ Inter Agency Standing committee of the UN, Approved guidelines on the CAP, paraBl, 1994 
nombreuses réunions. Cette confusion entre la récolte de fonds et la planification commune n'est pas sans poser problèmes. La première prend le pas sur la seconde et met en danger le processus de coordination en y introduisant la compétition sur le financement. Ainsi, les querelles sur la constitution des différents organes du PCP sont en partie liées à la crainte que ceux-ci n'évoluent en organes de sélection de projets. Pour certains, cette tâche est dévolue aux bailleurs de fonds qui doivent assumer leur responsabilité et non pas s'en remettre aux ONG ou aux Nations Unies, loin de renforcer la collaboration un tel processus la met en danger puisqu'elle place les différents acteurs de l'aide en concurrence. Ainsi, lors des années précédentes UNOCHA a tenté d'imposer aux ONG des modifications de leurs projets parce qu'ils ne correspondaient pas aux stratégies qu'il proposait, où encore de réduire les montants demandés.

\subsubsection{Les résultats de l'Appel}

Pourtant, les résultats en terme de récoltes de fonds sont loin d'être satisfaisants. L'Appel Consolidé ne couvre en 1998 qu'un tiers des dépenses réalisées par l 'ensemble des agences mais $2 / 3$ de celles de 1'UNOCHA. En 1998, $32 \%$ du budget total des projets mis en œuvre figuraient dans l'Appel ; au total 33\% des fonds demandés ont été obtenus. En septembre 99, le taux de couverture des demandes étaient globalement de 30\%. Si ces chiffres sont mis en avant comme étant le meilleur résultat enregistré par un Appel Consolidé onusien, ils ne reflètent cependant pas l'efficacité de cet outil en terme de récolte de fonds. En effet, de nombreux projets inclus dans l'Appel auraient été financés qu'ils y figurent ou non, les bailleurs de fonds ne l'utilisent pas loin s'en faut comme unique instrument de sélection des projets. Tel est le cas des projets financés par l'Union Européenne, qui n'impose pas aux ONG de faire figurer leurs projets dans l'Appel. Pour les donateurs qui en font une condition de financement : Les Pays Bas, Le Royaume Uni, Les Etats Unis, c'est davantage une formalité qu'un outil de sélection. Les relations entre bailleurs de fonds et leurs partenaires se font toujours sur un mode bilatéral, à cet égard le taux de couverture des projets classés prioritaires en 99 est révélateur puisque seuls $28 \%$ des priorités ont été financés. Comme le fait remarquer Antonio Donini, "les donateurs nous demandent de fixer des priorités mais continuent à financer leurs ONG ou leurs projets fétiches". Certaines ONG expriment leur lassitude face à un processus représentant une charge de travail supplémentaire mais peu fructueux et menacent de ne plus y prendre part. Cependant, la majeure partie des organismes tient à voir figurer leurs projets dans l'Appel que ce soit imposé ou non par les donateurs, il s'agit de ne pas être à l'écart d'un processus en vogue.

\subsubsection{La définition de stratégie commune : un véritable faux semblant}

Nous allons ici retracer la préparation de l'Appel 2000 afin de déterminer s'il représente ou non un premier pas vers une planification commune.

Le mécanisme se met en branle durant l'été, une série de groupe de travail est organisée dans les différentes régions pour ranimer le processus du PCP et les structures régionales afin de préparer l'Appel. Il faut rappeler que les organes de coordination régionale avaient dans le schéma initial un rôle primordial à jouer dans l'élaboration de stratégies et la définition des priorités régionales. Cependant, peu d'entre eux fonctionnent effectivement. Ce travail n'a pu être réalisé et la préparation de l'Appel se fait, hormis dans le cas du Hazarajat, en complète déconnexion des mécanismes de coordination régionale. 


\section{- Les groupes thématiques}

Les groupes thématiques ${ }^{50}$ organisés par UNOCHA sont chargés de l'élaboration du document de l'Appel 2000. Les projets sont classés depuis l'Appel 98 selon les cinq grands objectifs définis dans le cadre stratégique. Ces 5 thèmes sont plus un alibi, qu'un véritable classement opératoire ; ils ne reflètent pas le cadre de réflexion des acteurs, il s'agit de mettre en valeur des thèmes accrocheurs. Les thèmes sont les suivants :

- Allègements des souffrances humaines (activité d'urgence)

- Protection et promotion des droits de l'homme

- Fourniture de services sociaux de base

- Renforcement de la capacité des Afghans à construire des moyens d'existence durables y compris des alternative à la culture de l'opium

- Rapatriement

Les réunions de ces groupes ont un double objectif : élaborer une planification commune du travail des agences et ONG représentées et définir des critères de sélection des projets qui correspondent aux priorités. En septembre, sont organisées les réunions des groupes. Il faut noter que le temps imparti à la préparation de l'Appel est très court puisque les réunions commencent le 8 septembre et que les priorités doivent être diffusées entre les acteurs le 30/9. Par ailleurs, l'ensemble des projets doit être soumis avant le 15/10. Ces impératifs de temps sont dus à des contraintes liées au fait que l'Appel doit être prêt en même temps que l'ensemble des Appels des Nations Unies qui sont publiés fin novembre. Il est répété à tous ceux qui s'inquiètent du caractère trop court de ces délais ne permettant pas une véritable réflexion sur les priorités que la date butoir est fixée indépendamment de la volonté des acteurs. Mais, il n'est jamais expliqué pourquoi sa préparation ne commence pas plus tôt dans l'année.

Ce processus est déconnecté du travail interne des organisations participantes, chacun ayant déjà élaboré son projet et défini les objectifs, les réunions sont moins l'occasion de partages d'expérience et de réflexion qu'une tentative conciliatrice, si bien que les stratégies proposées sont au pire des textes vagues et consensuels ${ }^{51}$ définissant des objectifs tellement larges qu'ils pourraient être appliqués à n'importe quel pays en voie de développement; au mieux des textes définissant des bonnes stratégies en vue du thème mais qui ne sont pas nécessairement en lien avec les projets qui seront inclus dans le groupe. En effet, chaque ONG et agence choisit individuellement $d$ 'inscrire ses projets dans tel ou tel thème. Nonobstant le caractère superficiel de ce processus, certains se félicitent des progrès réalisés.

Il convient en outre de s'attarder sur les fréquentes allusions qui sont faites aux attentes des donateurs. Il semble intégrer par chacun qu'il ne s'agit pas de convaincre les donateurs du bien fondé de son projet mais de produire un programme qui corresponde à leurs attentes. " Il faut définir les priorités parce que les donateurs nous demandent de le faire ». A cet égard, le choix des thèmes porteurs tels que les droits de l'homme ou le renforcement de la capacité des Afghans à construire des moyens d'existence durables y compris des alternative à la culture de l'opium est parlant. La culture du pavot est considérée comme un thème transversal et les groupes s'efforcent de mettre en avant leur possible contribution à sa réduction. Autre thème récurrent, car médiatique celui de la femme, la " gender advisor » des Nations Unies est présente dans la plupart de ces réunions et plaident à l'envi la cause des femmes insistant sur leur prise en compte, dans les programmes, mais ceci ne débouche en aucun cas sur une réflexion sur la

\footnotetext{
${ }^{51} \mathrm{Cf}$. annexe ${ }^{\circ} 9$
} 
manière de mieux répondre aux besoins des femmes vulnérables. Le seul apport consiste à ajouter dans les textes au mot Afghan la mention «hommes et femmes inclus ». On recommande à chacun de ne pas insister sur les infrastructures parce que les «donateurs en ont assez de financer des infrastructures», il faut se concentrer sur ce qui leur plait : la dimension humaine, le travail avec les communautés, (en revanche il n'est à aucun moment défini ce qu'est une communauté en Afghanistan).

\section{- L'impossible définition de critères de sélection des projets.}

La deuxième phase du consensus est la sélection des projets prioritaires, les groupes thématiques n'étant pas parvenus à une exception prêt à définir des critères, cette tâche est remise à une réunion inter groupes thématiques, mais une fois encore aucun accord n'est atteint. Plusieurs raisons à cela, tout d'abord la concurrence entre les acteurs, chacun refusant toutes décisions qui risqueraient d'évincer ses projets; ensuite le manque de temps et enfin la composition des groupes. Il est difficile de voir un véritable débat à l'œuvre même a l'intérieur de petit groupe, les Afghans ont d'autant plus de mal à faire entendre leur voix, certains expatriés s'octroient le monopole de la parole, le problème de la langue ne facilite pas la communication. Il existe un réel besoin de développer les capacités d'écoute des personnes en charge de la coordination.

\section{- La sélection}

La troisième étape consiste donc en la réunion de comités restreints, nommés par chaque groupe, chargés de la lourde tâche de sélection des projets prioritaires. Nous illustrerons ce processus a travers l'exemple de la réunion du groupe sur l'amélioration des moyens d'existence. Celle-ci débute par des interrogations sur le mandat du groupe à déterminer au nom de tous des critères de sélection que personne n'a eu la volonté de définir auparavant, en outre la question de la compétence des personnes présentes qui en dépit de leur qualité ne dispose pas des moyens de pouvoir juger de projets qui recouvrent tous les secteurs et toutes les zones géographiques. Une proposition de refuser, par honnêteté de faire en deux jours une sélection parmi les plus de 150 projets soumis ne fait pas l'unanimité. En effet, chacun est conscient que si le groupe ne fait pas de choix celui-ci sera le fruit d'une décision arbitraire d'une personne en dernier ressort. Constatant que les bailleurs de fonds ne suivent pas les priorités, il est décidé de s'en tenir aux critères fort vagues $^{52}$ fixés par le groupe thématique, ensuite les fiches résumées des projets sont passées au crible, en une journée. Malgré cela la subjectivité des personnes influence les décisions prises. Ainsi un projet mal écrit aura plus de chances d'être retenu s'il est présenté par une agence des Nations Unies que par une petite ONG inconnue. Cependant, afin de limiter cet effet pervers, la majeure partie des projets est classée priorité 1.

Cette narration certes fastidieuse nous a semblé nécessaire afin de ne pas tomber dans le piège des discours ambiants louant les progrès réalisés depuis quelques années dans l'élaboration du l'Appel Consolidé et notamment la mise en avant des priorités, Comme le fait remarquer l'un des participants : « le nombre d'heures et le volume d'argent dépensé ${ }^{53}$ dans la préparation de l'Appel est considérable mais cela n'a aucune conséquence sur le travail de terrain. Il est très facile de se faire prendre au piège et de penser que cela est important alors que cela ne l'est pas du tout. " Le document de l'Appel est utilisé comme un instrument de promotion du PCP, faisant croire qu'une réelle planification commune est à l'œuvre alors que ce n'est qu'une pseudo-consultation totalement détachée des réalités du terrain. Les fondements

\footnotetext{
52 les critères sont la pertinence du projet en fonction du thème du groupe

${ }^{53}$ Frais de déplacement, location du Marriott hotel, temps consacré par les directions des agences et des ONG.
} 
mêmes du système sont douteux, il permet à quiconque d'inscrire ses projets puis impose aux acteurs eux-mêmes de sélectionner les projets en fonction de stratégie dont on a vu qu'elles étaient peu pertinentes. Cette définition de priorités imposée par les donateurs n'est pas un outil permettant une meilleure efficacité de l'aide.

\subsection{Un bilan mitigé}

Comme nous l'avons constaté les fruits du processus de coordination se font encore attendre aujourd'hui en Afghanistan, après de longs débats sur les questions de structures, on se rend compte aujourd'hui que ceux-ci n'ont pas permis la mise en place d'organes efficaces, c'est le cas de l'organe national de coordination dont le bilan après un an de fonctionnement n'est guère positif, et sans vouloir faire de prospective pessimiste il est à craindre que les organes régionaux de coordination ne se trouvent confrontés aux même écueils, les problèmes de fond n'ont pas été abordés. Il est à craindre que l'abus du droit de représentation démocratique ne conduisent là aussi à une paralysie des organes.

Le seul résultat visible du processus à savoir l'appel consolidé n'est qu'un faire valoir, mais en rien le signe d'une meilleure coordination sur le terrain.

Cependant, il ne faut pas omettre du bilan les avancées intangibles, la majeure partie des personnes interrogées affirment que la situation est largement améliorée si l'on compare à ce qui se passait en 1996, le principal étant un changement dans les mentalités des acteurs de l'aide, beaucoup admettant aujourd'hui qu'une meilleure coordination est nécessaire que ce soit au sein des différents groupes d'acteurs ou entre les groupes. Les personnes se rencontrent se parlent lors des réunions, des liens se créent, et si le vieil antagonisme Nations Unies / ONG persiste, des actions en commun ont été menées.

Une étude des progrès réalisée à l'aulne de l'état de la coordination avant le début du processus permettra ainsi d'avoir une vision plus optimiste des résultats. Le climat de compétition exacerbée ouverte ente les agences des Nations Unies s'est estompé, le processus s'est accompagné d'un certain renouveau du personnel notamment expatrié, ainsi les directions de UNICEF, de l'OMS et du PAM ont été renouvelées sous la pression des donateurs. Un véritable processus de restructuration est à l'œuvre actuellement au sein du PAM, visant à démembrer les réseaux informels qui entravaient son action. A Jalalabad, plusieurs agences ont ouvert des bureaux communs, limitant la duplication des frais administratifs. Ce sont certes des avancées limitées mais qui représentent peut être un premier pas.

Chacun estime qu'il est aujourd'hui nécessaire d'évaluer le processus dans son ensemble, il est cependant révélateur qu'aucune évaluation officielle n'ait été engagée. La mise en place du système d'évaluation a été retardée en raison de soit disant problème de recrutement. Les fonds destinés à ce volet du PCP sont aujourd'hui utilisés pour réaliser des bases de données régionales censées servir de base aux futures évaluations.

Les raisons évoquées par le personnel de l'UNOCHA pour expliquer le peu d'évolution sur le terrain sont essentiellement les aléas des conditions de sécurité. Ainsi Antonio Donini explique que l'absence de fonctionnaire international sur le terrain, dans certaines régions, notamment de coordinateur régional explique en large partie le peu de résultat. L'étude de la région choisie permet cependant de nuancer cette position. Si on ne peut que constater que le processus est stagnant dans l'Est, rien ne prouve que la présence d'un coordinateur régional suffirait à le réanimer à contrario, l'exemple du Nord illustre le fait que l'existence d'un 
coordinateur régional n'est pas une condition suffisante à une meilleure coordination. Dans ce cas, on invoque le fait que le processus ne peut être parfait une fois encore en raison des conditions de sécurité qui ne permettent pas sa présence permanente sur le terrain. L'analyse du Hazarajat montre que celui-ci ne peut pallier tous les obstacles à une bonne coordination.

Il semble qu'évoquer les questions de sécurité comme un frein au processus, revient à poser le problème à l'envers. Certes, suite aux bombardements américains d'août 98 et à l'assassinat d'un employé des Nations Unies à Kaboul, l'ensemble des expatriés des Nations Unies a évacué l'Afghanistan. Les organes régionaux de coordination qui venaient d'être formés n'ont donc pu fonctionner normalement. Mais dans un contexte tel que le contexte afghan, n'est il pas plus sage d'envisager un système qui fonctionne dans des conditions défavorables ? Cette évolution montre la vulnérabilité du système onusien et son inadaptation aux crises complexes. La présence de personnels expatriés des Nations Unies est considéré comme un élément indispensable auxquels aucun palliatif n'a été envisagé, en raison du manque de formation et d'implication du personnel local et du manque de confiance envers les autres acteurs notamment les ONG. Celles-ci, confrontées à des conditions de sécurité moins draconiennes que les agences onusiennes sont sans doute mieux à même d'assurer le suivi du travail effectué sur le terrain et d'une éventuelle coordination. Les problèmes de sécurité loin de permettre un rapprochement entre les deux catégories d'acteurs ont renforcé le fossé préexistant. 


\section{CONCLUSION}

Cette étude n'est qu'une ébauche. Elle aura atteint ses objectifs si elle permet aux lecteurs de comprendre la complexité des enjeux qui sous-tendent le processus de coordination et de l'architecture mise en place. Cette tentative est beaucoup plus qu'une réforme purement technique d'un mécanisme de coordination. Elle procède d'un mouvement qui dépasse le cadre de l'assistance à l'Afghanistan et dont les enjeux sont les rôles et l'importance relatifs des différents acteurs de l'aide.

Est-ce à dire qu'il faille abandonner le processus?

$\mathrm{Si}$ aucun progrès n'est réalisé en terme de coordination, si la configuration actuelle est maintenue, si elle constitue un substitut à la nécessaire réforme des Nations Unies, si elle contribue à déresponsabiliser les Etats de la résolution des crises, si la consultation conserve son - caractère fallacieux, si l'assistance n'est plus qu'un agent aux mains des Etats qui la financent, si les structures choisies n'entraîne que la paralysie du dialogue..., La réponse est sans nul doute oui.

Or l'analyse du processus a montré que ces effets pervers sont déjà perceptibles. Il faudrait donc arrêter là une initiative dangereuse pour l'avenir des actions de solidarité. Cependant, le rejet pur et simple de ce processus ne semble pas une réponse adaptée, les risques analysés ne tiennent pas seulement au PCP. Il convient donc d'y apporter une réponse que ce soit ou non dans le cadre de ce mécanisme. Notre propos n'est pas ici de trancher s'il sera ou non plus productif de profiter des espaces créés et des fonds dégagés pour tenter de mettre en œuvre une coordination effective et efficace. Mais il semble si l'on tient compte du temps et de l'argent qui y ont été investis, qu'il soit plus aisé de tenter d'influencer le processus de l'intérieur qu'en se tenant à l'écart.

Cette initiative venue des sommets ne portera ses fruits que si elle est réappropriée par ceux qui sont quotidiennement aux prises avec les réalités du terrain et au-delà par les populations concernées. Si la population afghane est quasiment absente de cette analyse ce n'est que le reflet d'un processus qui tend à occulter les destinataires de l'aide. Il est pourtant fondamental de les impliquer dans la définition des objectifs et des priorités de la communauté d'aide.

La tendance actuelle doit être inversée au profit d'une approche réaliste des problématiques afghanes. Il convient de fixer des objectifs plus précis que celui de contribuer à la construction de la paix. La réflexion doit s'appuyer sur l'expérience du terrain et sur les potentialités des acteurs.

Or, les ONG mettent en œuvre la majeure partie de l'assistance à l'Afghanistan et possèdent sans doute une plus grande connaissance du terrain. Elles doivent donc davantage s'impliquer et jouer un rôle à la mesure de leur engagement sur le terrain. Pour ce, il faut avant tout qu'on leur en donne les moyens. Mais elles doivent s'organiser et cesser de crier à la manipulation et à la préservation de leur sacro-sainte indépendance, qui n'a pas de valeur en soi mais parce qu'elle permet de mieux répondre aux besoins des populations. Elles doivent certes rester vigilantes afin d'éviter les écueils d'un fonds commun et de la conditionnalité de l'aide, 
mais aussi faire la preuve de leur capacité à se coordonner et à dialoguer avec les donateurs afin de mieux diffuser leurs analyses. Plutôt que d'adopter une attitude d'opposition frontale aux Nations Unies elles doivent faire la preuve qu'elles sont capables de prendre en main la coordination. L'avenir de la coordination dépend en partie de leur mobilisation, que ce soit au sein ou en dehors des structures mises en place dans le cadre de la programmation commune. 


\section{BIBLIOGRAPHIE}

\section{Ouvrages:}

- J.Bennet NGO coordination at field level, International council of voluntary agence, Oxford 1994

- Bertrand Badie \& Marie Claude Smouts: Le retournement du Monde, Presse de la fondation nationale des Sciences politiques, St just, 1995

- Maurice Bertrand: "The United Nations : Past, Present and Future" Kluwer Law International, The Netherlands 1997 ,

- J-P Deler, dir. Y-A Fauré, A Piveteau et P-J Roca, ONG et développement - société, économie, politique, ed.Karthala, Paris, 1998.

- Donini The policies of mercy: UN coordination in Afhganistan, Mozambique and Rwanda, DH 1996

- M. Keating « Aid politics : dilemnas of humanitarian assistance » in Essential field guide to humanitarian and conflict zone : Afghanistan, ICRC, Geneva 1998

- Jean-Christophe Rufin: "Le piège humanitaire",Pluriel, Paris, 1994

- Anders Davidson and Peter Hjukström; "Afghanistan, aid and the Taliban”, S.C.A. Swedish Committee for Afghanistan Stockolm, 1999

- «Database of the Ngo activity, 1999 », ACBAR, Peshawar, 1999

- "Directory of humanitarian agencies working in Afghanistan", ACBAR,Peshawar, 1999

\section{Revues:}

- Strand, S.Barakat : « rehabilitation and reconstruction of Afghanistan a challenge for Afghans, the NGO and the UN », disaster prevention and management, vol. 4, n. 1,1995 , university press.

- Helga Baitenmann : « NGO and the Afghan war » thirld world quaterly, vol.12, n. 1 ,janvier 90

- G. Dorronsoro ; «Les enjeux de l'aide en Afghanistan »p93-112, in Culture et conflits n 11, automne 1993, L'Harmattan.

- P. Newberg "Politics at the heart. The Architecture of humanitarian assistance to Afghanistan". International migration policy program, n2, july 1999-11-17

- P. Salignon, P-P. Vandini ; "quelle coordination?", Les nouvelles d'Afghanistan, p $3-4, n 84,1^{\text {er }}$ trimestre 1999

\section{Rapport :}

- P. Wiles Dir. « Evaluation of Danish assistance to Afghansiatn 1992-98 »1 ${ }^{\text {st }}$ draft,ODI, London, 1999

- A Strand, A.Najimi, N. Lander « NGO cooperation open for debate, interim report and preliminary recommandations » commissioned by ACBAR, ICC ANCB, Peshawar 1999 
- A Strand, A. Najimi, N. Lander «NGO coordinaion in Afghanistan, an evaluation report » commissioned by ACBAR, ICC ANCB. Peshawar 1999

- A Witschi Cestari « Coping with complexity » mission report,UNOCHA, 1998

- Dir. M.Keating « Making a reality of common programming », UNOCHA, Islamabad, 1998

- Don Sauer « the consequence of PCP for Afghan NGO », ANCB, Peshawar, 1998

- S.V. de Mello « Mission report on Afghanistan ». New York, March 1998

- Donini «Afghanistan, coordination in a fragmented state », DHA, New York, 1996

- G. Dorronsorro ; "Afghanistan la nouvelle domne » in rapport d'activites Afghanistan1992, A.M.I., Paris 1993

- L. Frechette « Strategic Framework for Afghanistan »,OCHA, New York, 1998

- collectif « responses to the making a reality document » UNOCHA, Islamabad 1998

- O. Scherrer "Rôle de l'aide internationale dans le processus de reconstruction de l'Afghanistan 1999-93", memoire de recherche en Sciences sociales, Université Lyon2,1996

\section{Compte-rendus de réunions et discours}

- J.Bennet « Afghanistan : coordination of private aid agencies », ,ACBAR,Peshawar, 1991

- Les comptes rendus de l'ensemble des réunions de l'Afghan Task Force, UNOCHA, Islamabad 1996-1998

- Les comptes rendus de l'ensemble des reunions de l'Afghan Support Group, UNOCHA, 1997-1999

- Les comptes rendus de l'ensemble des réunions de l'Afghan Programming body, Islamabad, UNOCHA 1998-99

- Les comptes rendus des réunions de coordination des régions étudiées.

\section{Communiqué de presse :}

- UN Secretary General « The humanitarian challenge today », Los Angeles, 21/04/98 


\section{TABLE DES ANNEXES}

Annexe 1: Abréviations

Annexe 2: Liste des personnes interrogées

Annexe 3 : Cartes

1. L'Afghanistan Relief

2. Le découpage régional alternatif à la proposition de l'UNOCHA (source SCA, fax faf/mk/002, May 98 - Peshawar)

Annexe 4: Financements de l'Aide en Afghnaistan

1. Evolution des fonds des $\mathrm{ONG}$ (source : ACBAR - directory 1998, p. iv, ACBAR Press, Peshawar 1999)

2. Contribution des donateurs

3. International assistance (source : UNOCHA - Afghanistan Appeal 1999, p. 18\&19, Islamabad 1998)

Annexe 5: Chronologie

Annexe 6: L'activité des coordinations

General discussions on role and achievements of coordinating bodies (source : A. Strand - NGO coordination an evaluation report, ACBAR, Peshawar 1999)

Annexe 7 : Making a Reality of Principle Common Progarmming

1. Résumé de texte

2. Structure. (source : Making a reality of Principle Common Programming)

Annexe 8: Cadre stratégique

Political Strategy. (source : Nations Unies - Strategic Framework for Afghanistan, August 1998)

Annexe 9: Term of Reference of Thematic Group : Return of Refugees

Source : IOM, fax 25/10/99

Annexe 10: Exemple de stratégie de groupe thématique

Source : S. Yannoutsou, TG empowerment of Aghans to build sustainable livelihoods, October 1999 
Annexe 1

Abréviations 


\section{ABREVATIONS}

- ACBAR : Agency Coordination Body for Afghanistan

- ACC. : Adminstrative Committee on Coordination

- AETF : Afghan Emergency Trust Fund

- ANC.B : Afghan Ngo Coordination Bureau

- ASO : Area Security Officer

- ATF : Afghan Task Forces

- ASG Afghan Support Group

- APB : A fghan Programming Body

- BAAG British Agency Afghanistan Group

- CG ; Co-ordinating Group

- -CAS : Common Assistance Strategic

- DFID Department For International Development (aid GB)

- DPA : Department of Political Affairs

- ECHA : Executive Communitee on Human Affairs

- ECHO European Community Humanitarian Office

- FACINGO Funding and Capacity International NGO

- FAO Food Aid Organisation

- GOA : Government of Afghanistan

- ICC : Islamic Coordination Council

- IDP Internally Displaced Person

- IGP : Income Generating Projects

- ILO: International Labour organisation (BIT)

- IOM International Organisation for Migration

- KRG : Kaboul Relief Group

- MACA : Mine Action Center for Afghanistan

- MAPA : Mine Action Program for Afghanistan

- MOPH Ministry Of Public Health

- MOU Memorandum Of Understanding

- NCB Ngo Coordinating Board

- NID National Immunisation Day

- OIC Organisation of Islamic Conference

- PROMIS : Program Managment Information System

- PEACE : Poverty Eradication and Community Empowerment

- QIP Quick Impact Projects

- RCB Regional Coodinating Body

- RCO : Regional Coordinating Office/ officer

d Resident coordinaling Officer (islamabad)

- RRD Rural Rehabilitaion Departement

- TOR : Term of Référence

- TWG Tuchnical Working Group 
- SMC : Strategic Monitoring Committee

- SWABAC : South-West Afghan Baluchistan Associatinfor Coordination

- TG : Technical Group

- TWG Thematic Working Group

- UNDAC UN desaster assessment and coordination

- UNOPS ; UN Office of Projects Services

- UNPI UN Public Information

- UNSMA : UN Special Mission Afghanistan

- UNDP : UN Development Program

- UNDHA : UN Department of Humanitarian affairs 
Annexe 2

Liste des personnes interrogées 


\section{LISTE DES PERSONNES INTERROGEES}

\section{Bailleurs de fonds}

E. Mac Leod, programme coordinator, , Peshawar office

Tom Hushek, coordinator for refugee affairs, Embassy of the United states of America, Islamabad

O. Planson, premier secrétaire, ambassade de France, Islamabad

V. Carvell, counselor development, haut commissariat du Canada, Islamabad

Govert Wisser . Afghanistan Program, ambassade des Pays Bas Islamabad

M. Lindvall, ambassade de Suède, Islamabad

K. Lander ambassade de Finlande Islamabad

A. Robyns. ECHO, Islamabad office

M. Peterson, delegation of the European Union, Islamabad

Yamaguchi Shinobu, Political Attaché, embassy of Japan

\section{Agences des Nations Unies}

Jean Michel Arnaud, FAO, Peshawar

Anoja UNICEF, Jalalabad

Win Win Kayo, OMS, Jalalabad

Dr. Attiqullah, HCR, lalalabad

M. Semple regional coordinator officer central region

Marvyn, regional coordinator officer northem region

A. Donini, Deputy humanitarian coordinatorUNOCHA Islamabad

S. Yanoutsou, Deputy resident representative, UNDP,

M.A Sackett, WFP representative, Islamabad

S. Reynolds. Habitat representative, Islamabad

\section{O.N.G.}

JF. Cautain directeur des programmes MADERA, Peshawar

C. Mac Fadden, executive director, ACBAR, Peshawar

N. Harild director, DACAAR, Peshawar

Dr Anwarulhay, director general, Ibn Sina, Peshawar

M. P. Caley, ACTED, Paris

Louise, MSF holland, Peshawar

Christy Scott, IRC, Peshawar

TIM \& Di Abdul Hadi Health net, Jalalabad

Erhart German Agro Action, Jalalabad

D. De Poerck, Programme director, Health net international, Peshawar

Laurent. Solidarité, Bamyan

Ramazan, regional coordinator Hazarajat, Madera

S. Worsley, program director care international, Peshawar

Eng Zia UI Haq, CC.A, programme manager, Peshawar

Latifi, Marut, Peshawar

doctor Sarvar Hemati ,SCA, Jalalabad

Dr Jalal IBNSINA Jalalabad 


\section{Annexe 3}

Cartes

\section{L'Afghanistan Relief}

Le découpage régional alternatif à la proposition de l'UNOCHA 


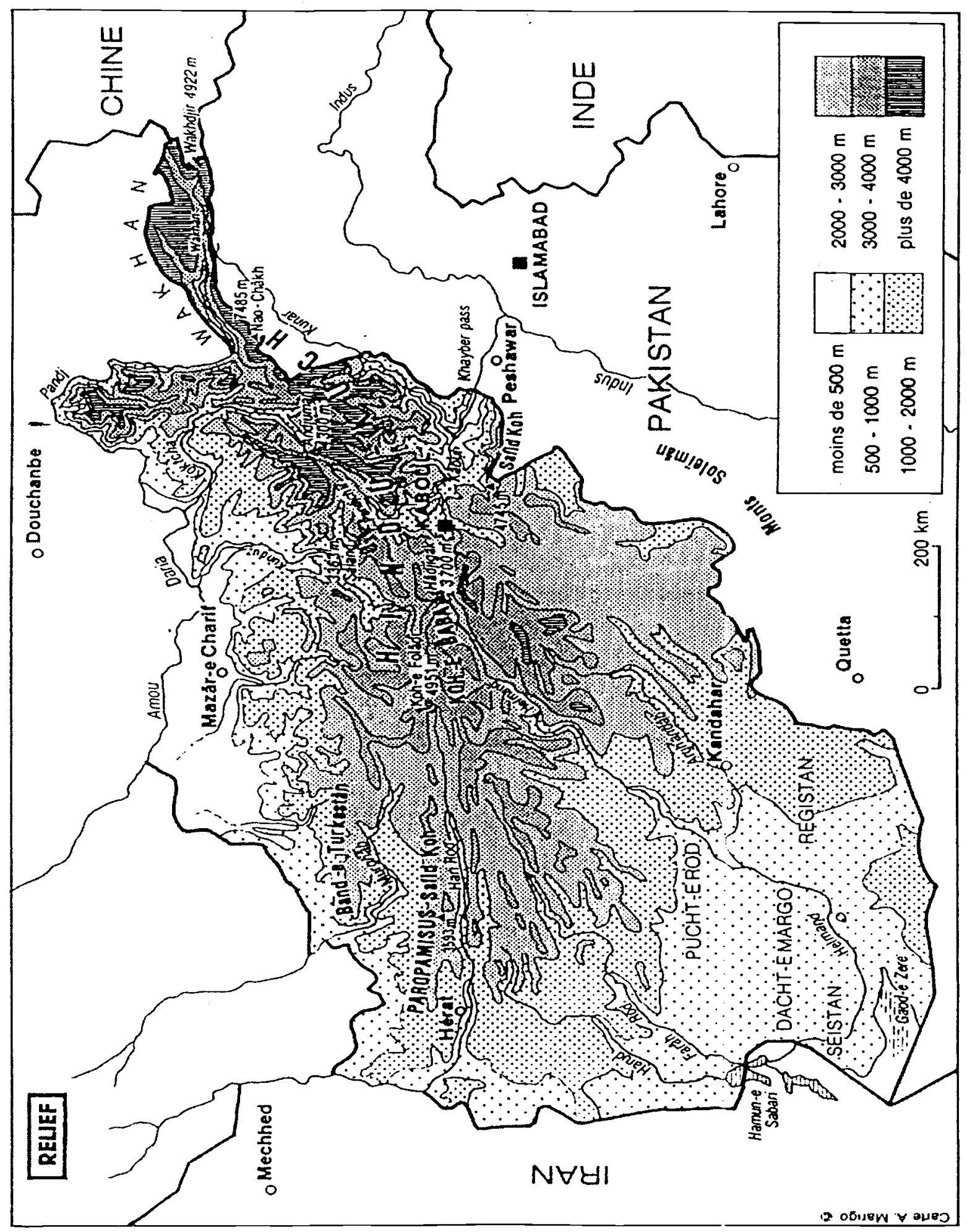




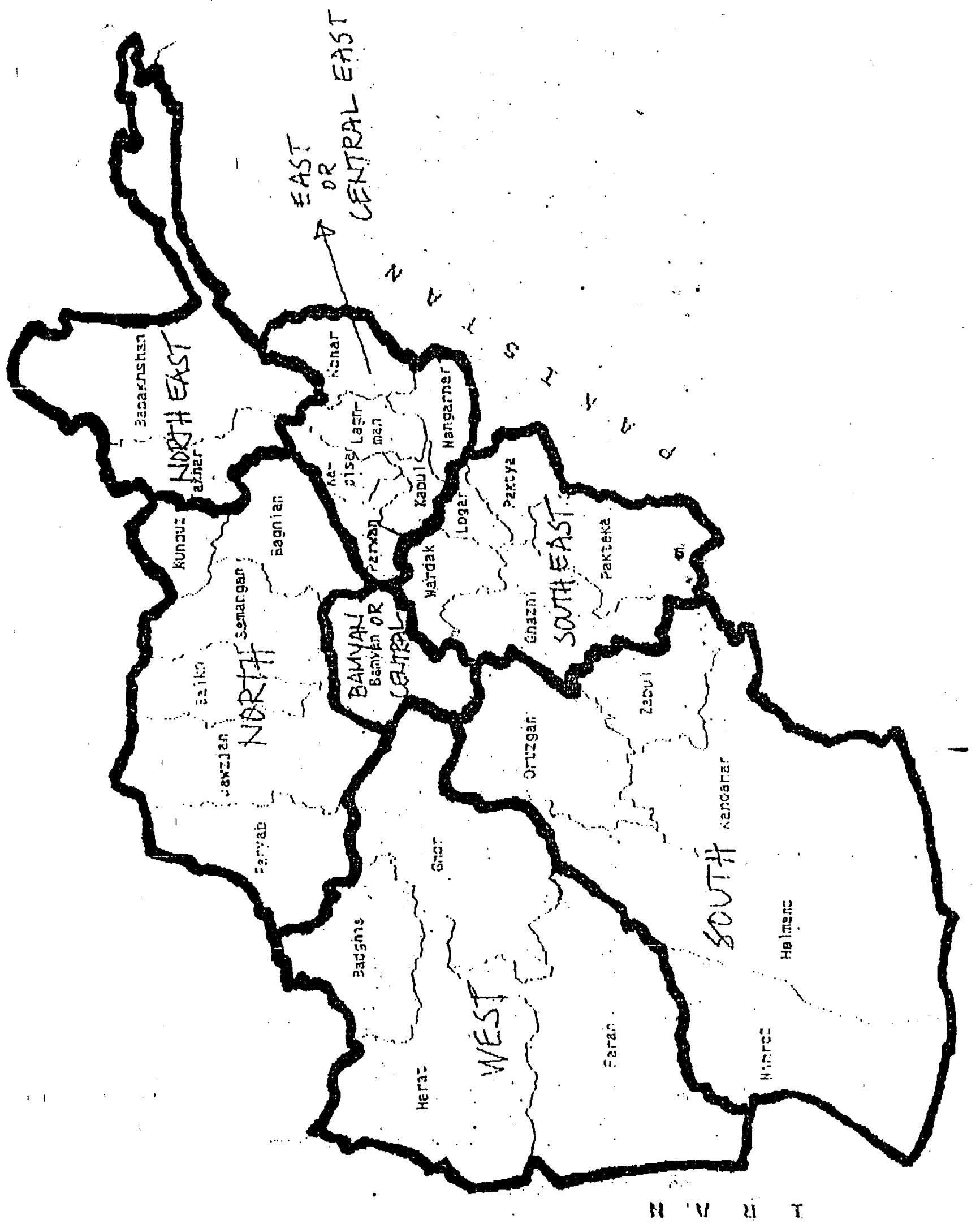


Annexe 4

Financements de l'Aide en Afghanistan

1. Evolution des fonds des ONG

2. Contribution des donateurs

3. International assistance 
TOTAL BUDGET EXPENDITURE 1993 - 1998

(US \$ MILLION)

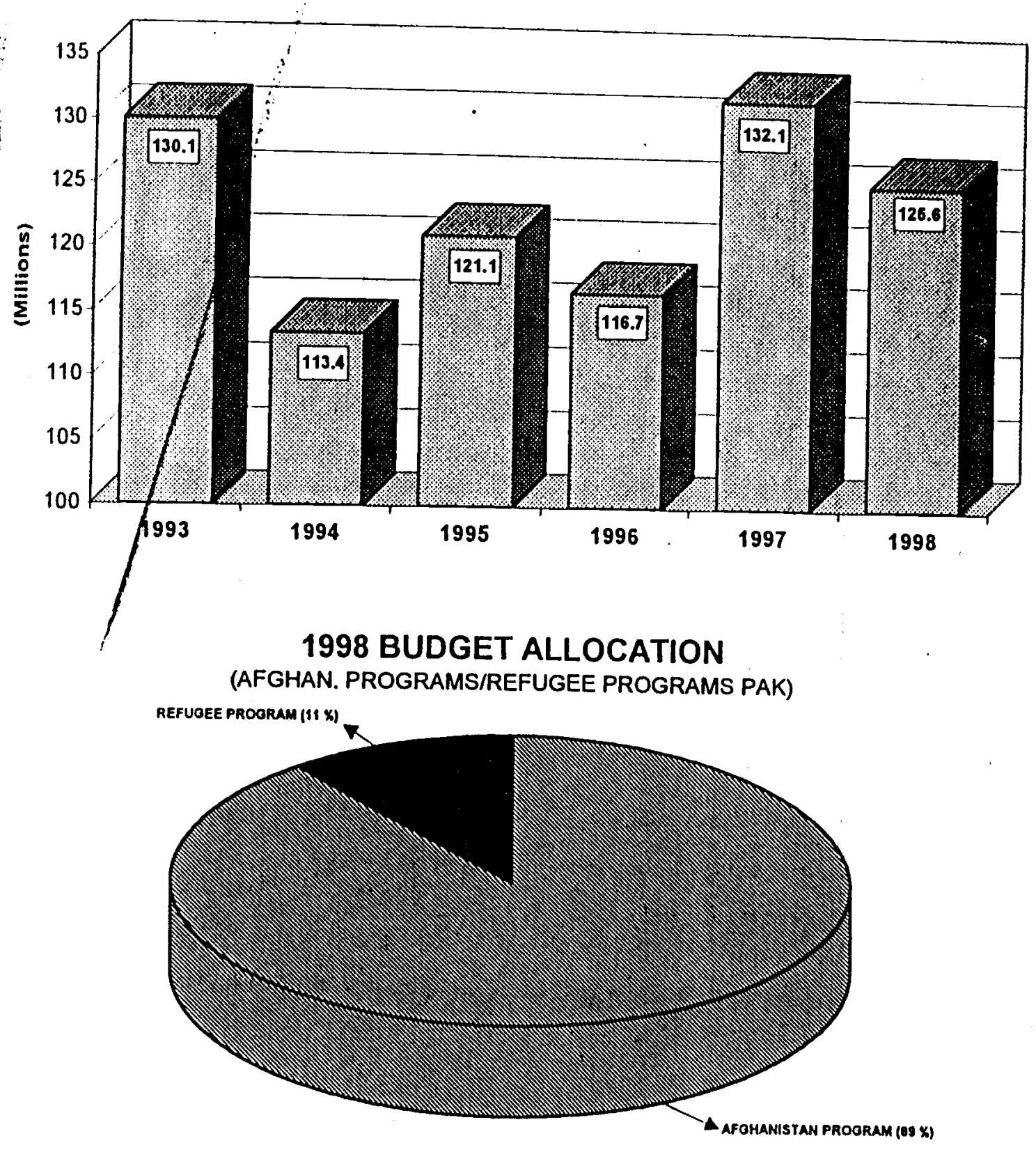

THE FIGURES IN THESE TABLES, GRAPHS RELATE ONLY TO ORGANIZATIONS IN THIS PUBLICATION 


\section{Donor Contributions}

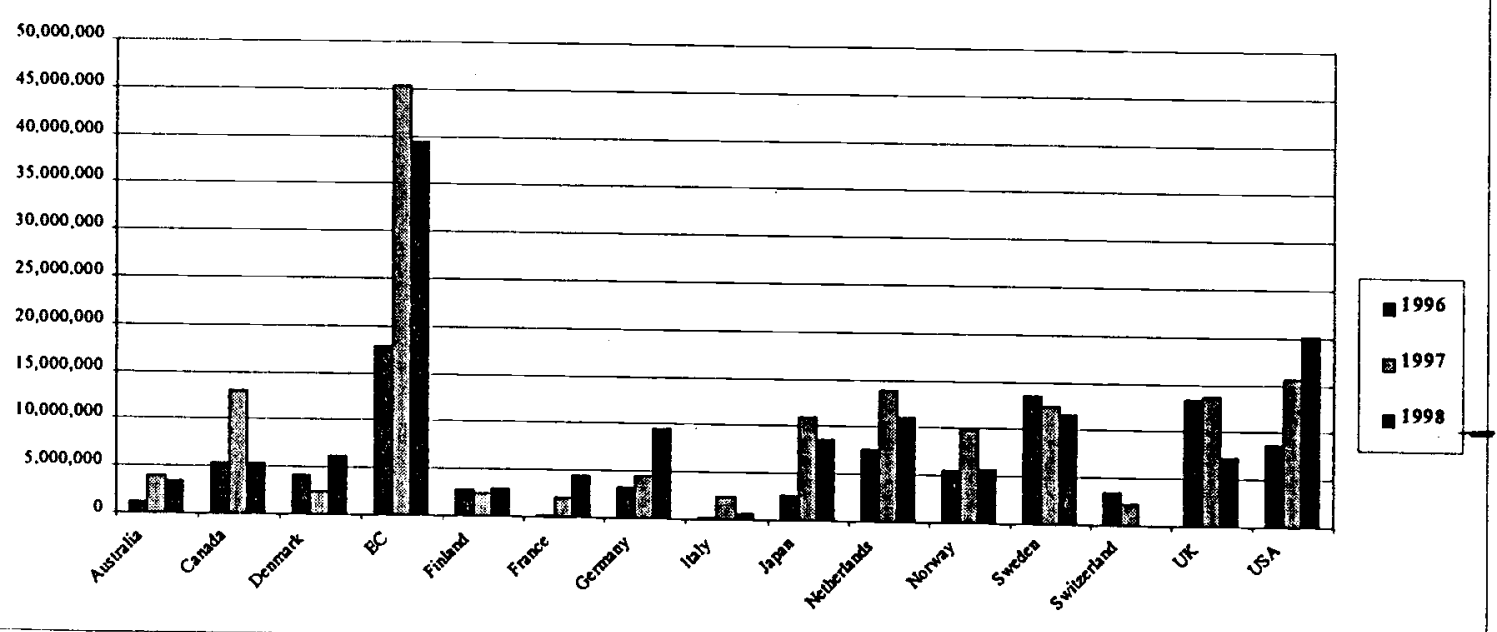


2111

1011

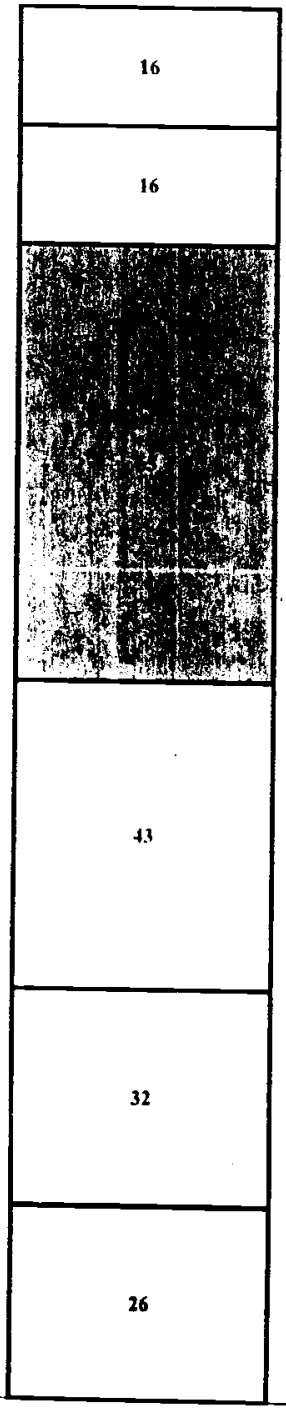

1946

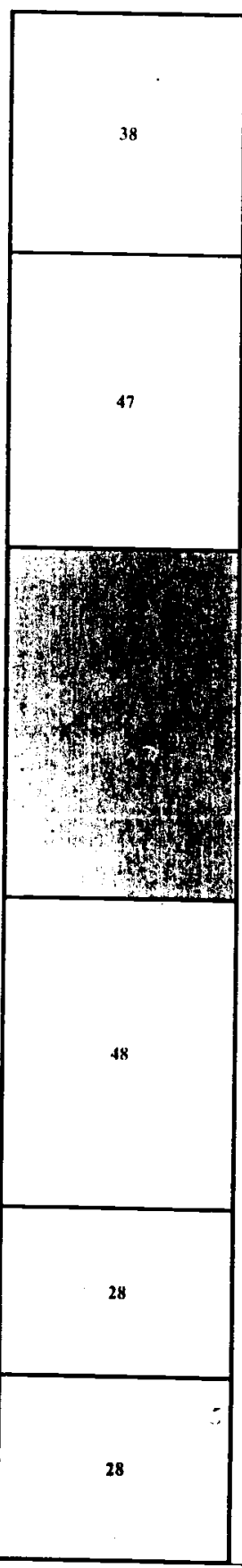

1997

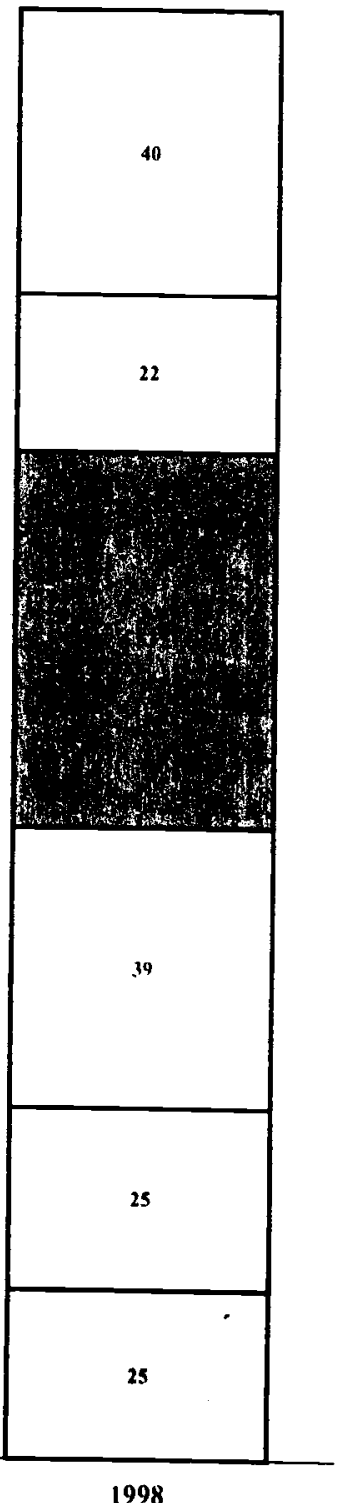

DICRC/IFRC

$\square$ Outside Appeal

Q Appeal

口WFP

$\square$ NGO Core Funds

$\square$ UN Core Funds

i:

N.B. NCiC ( ore lineds are based ou AC BAN data. ICRC/IFRC"s figures for 1948 are approvim 
Annexe 5

Chronologie 


\section{Summary of events : \\ Strategic Frame work and Principled Common Programming Process}

1995:

September :

- ACC Administrative committee on Coordination $8^{\text {th }}$ session of 95

UN highest coordinating body. this committee is composed of the Gecretary General and the heads of the secrebariats of the specialised agencies. of the lnternational atomic energy agency and of the Brettons woods organisations. Its task is to supervise the agreements made between the UN and its agencies and to ensure their coordination

It agreed on need for a study of UN system capabilities and modalities for post conflict reliabilitation and reconstnction and for the articulation of an integrated post conflict framework and strategy

1996:

June :

- Informal consultative meeting on coordination of assistance

Donors, UN, and NGOs

Under the pressure of ACBAR it agrees on the creation of Interagency Task Force to prepare appeal 97 in an integrated and panticipatony manner. The Task force includes Netherlands, the EC, Norway, ACBAR. ANCB. UNOCHA and UNDP

An international formm on assistance to Afghanistan will be held. the Task Force will also prepare it

- Mid term review consolidated appeal 96

July:

- New head of UNSMA UN Special Mission for Afghanistan Dr. Holl

September

- ACC, Administrative committee on coordination $9^{\text {th }}$ session of 96

It contirmed the need for an approach in dealing with crises environment

Consensus on the need to pre-tested agreed approaches in selected country

November

- Group of 21 New Yorli convened by the secretary gencral composed of countries of interest/influence in Afghanistan

December :

- Latunch of consolidated appeal 97: pledges 133 Million USD

1997:

January :

- New UN Secretary General Kofi Annam

- 21-22 International Forum on Assistance to Afghanistan in Ashgalbat

Panticipants: representatives of 27 countries (donors and neighbouring countries. the European Union.) NGO: (Alyhan: 18. international:20). coordinating body ACBAR, ANCB. SWABAC). ICRC. UN (DHA. Agencies headquaters and field staff. OIC. Asian development bank, world bank. joumalists

The aim was to bring together key players in the assistance to Afghanistan with a vicw to identify a common strategy:

Conclusion : the objective of international assistance to Afghanistan should be sustainable peace. Both culerelles :md development/rehabilitation activities must be implemented 
Common commitment to derelop a strategic framework based upon capacity building. strengthening local conom!. reducing human suffering and on prunciples (relating to human rights. the lumanitarian intperative. and the impantiality of aid.

Afghans full involvement in planning should be ensured.

The UN's presence in Afghanistan should be strengthened in view to cnlanncing the system coordination of like intcrnational commmunty as a whole

Comments Ashgabat is one of the starting point of the process. since it is there that the UN affirmed to the donors that they would take the leadership in assistance delivery in Afghanistan. Though they claim to have reached a consensus. very divisive issues were given a good airing but consensus was not given an onlcome.

Wharcis the aid community wishes to link political and assistance activities the UNSMA insisted on kecping them scparated.

The aid community was also divided on the approach to be taken to human rights and gender issues.

\section{March}

- i-7 Consultative committee on program and operational questions $10^{\text {Th }}$ session Geneva

II states that between September 95 and march 97 “ over 100 officials dranvn from UN system cntitics and the Brettons woods institutions have becn conducting an informal review on theses matters. It endorsed the report of Hugh Chomondeley' and the principle of the strategic approach in response to crises

"A stritegic framework aims at a more integrated. coherent and united approach to plaming the response to the emergency and facilitating recovery. all assistance actors must work in greater harmony"

Retict and development activities must overcome the existing divisions. The in-country UN agencies must reum to thein tormer position in the vanguard of international implementation effort.

Afghanistan belongs to the group of 25 countries experiencing complex crises.

\section{- 15 end of UN dual coordination:}

UNDP and UNOCHA bought together but adminstmation and budget remain separated

Appointment of a single UN resident coordinator in Islamabad Alfredo Witschi Cestari (former UN lımmanitarian coordintator

April:

- Group of 21 convened in New York by the Secretary General composed of countries of interest/infhience in Afghanistan

- $9 / 4 / 97$ Afwhan Task Forec UN/taleb relation a dev (see minutes of the meeting)

- $\quad 11-11$ Administrative committec on coordination $1^{\text {st }}$ session of 97 reallims the importance of partnership and alliances in crisis situations

It selects Afghanistan and Mozambique as test countries for Strategic Framework exercise. it further emphasised to take the gender perspective into account in testing the strategic framework exercise: Hugh Cliomondeley is assigned to formmlate the SFA

- I diụs Workshop Taliban anthorities/UN in Kandahar on all aspects of the UN's assistance including the issuc of women

- $\quad \because$ L'Afghin Tasli Force

It agrees to act in a slupervisory and advisory function to the SFA team.

The Afghan Task Force will be enlarged by the participation of WFP. WHO. UNICEF. The US. llic Uk and Sweden.

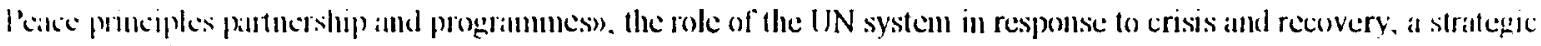
approach bl hush (homondeleyloy) 
- 21: lirst Mghan Sirpuror Girup in Geneval

I1 "1as a meeting of 14 donors set up by Minister Pronk. minister for Development and cooperation. Holland. UN agencies were invited but there was no NGO

tiili

Discussion on the link between political and aid activitics. It was agreed that the link between UNSMA and LINDP was vital and that relations between UN Agencies need to be strengthened. therefore Mr Holl UNSMA stresses that political and assistance activities have important differences.

Supports of the donors on the formulation of a strategic framework provided it is not too cimbersome and not 100 costly. hey are aware that it is a long process and that it can not be ready before the end of the year. They insist on the fact that Afghin. NGOs and donors ownership of the process is ensured. ciscler: donors accepted a degree of pragmatism but want to ensure that programs have no discriminatory eflect. they complaim abont some of the UN agencies being overly pragmatic.

The agrec to recomene this type of meeting

- cister commillco

M:1::

- presentation of the proposed Strategic Framework exercise to concerned actors in the field

- establishment in New York of the Ad-Hor interagency group on gender issues, an inter. agency lorum to discuss gender issue in Afghanistan. led by Angela King

- 5 lnforigency Task Forece

Agree 10 ace in a supervisony and advisory function to the Strategic Fantowork team

- Selection of the members of the SF team : led by Hugh Chomondeley(UN Staff college, Sultan A/1/ UNDP. Antonio Donini(DHA). Linda Perkins DPAn Steven Holzman World bank

- $12 / 1+\mathrm{U} / \mathrm{N} /$ taliban meeting Kandaloar on education (

- 25/28 U/N/taliban meeting Kandahar on licalth postponed

Junc

- 3 recommendations of UN Executive Committec on Humanitarian Affairs ECHA

It recommends a principled approach to the gender issue in Afghanistan and stated that UN and implementing partucrs will take a consislent approach

I N agencies will not engage in institution building efforts of the Afghan autority as long as their discriminaton practice continue

Heids of UN will no longer make milateral declarations on UN policies and practices relating to humlinn rights obsenance in Afghanistan

- Security Comeil presidential statement on "protection for hmmanitarian assistance to refugees and ollers in contlict sitmation): it " stresses the importance of ensuring closer cooperalion belween UN bolies and other international agencies."

- V'isil hy Hugh Chomondoley to the field

(1) prepare the Stralcgic Framework exercise. accompanied by Mr. Witschi-Cestari and Michact Keatmg

July :

- 2 Mlid term review meting. Islamaband

The Nethetiands complain on the Postponement of the Strategic Framework tean and commented on IIs ste and cost

- 17/17 New Term of Reference of Task Force adopted

Members: ANCB ACBAR. EC. the Netherlands. UK. USA. Sweden: UNICEF: WFP. WHO H( R.IINOCHA/UNDP

Ince Agenty Alghan Task Force became Alghan Task Force, it is an and hoc mechamism

It ams at cusumg halt Smategic Framework is in line with the basic international human rightes standards and that all aid practitioners in Afghanistan perform their duty accordingly.

tulumu: Germany. ICRC werc also member

August: 


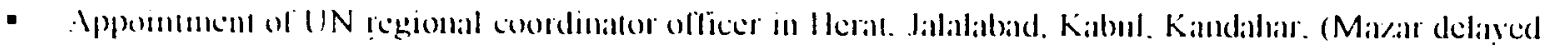
b) lighting).

- First drati on the formulation of a stralegic framework circulated among NGO and UN agencies

Septombere

- $\quad 52^{\text {t"l }}$ sicssion of UN Gencral Asscmbly:

Bohl humanitarian /economic and political activity are General assembly items: the political item dilles to the early sots when the security comeil was mable to act on that question of international peace and security owing to the negattive vole of a pertuancent member

- 19 Sieptember lo 15 October field mission of Strategic Frameworle team

led by Hugh Cholmondeley Including representatives from UNDPA (depantment for political alfairs: Linda Perkin. UNDHA Depantment for hmmanitarians allairs (Now OCHA): Antonio Donini : UNDP Lim! Deboice. World Bank: Sicie Holtzman: UNESCWA:T. Olaid. N. Stockton: OXFAM Larry Mincar UNSC + Koiling. P Newberg K Ostrom(UNOCHA)+ 2 WB from Islamabad.

- Joint technical commission between UN and Taleh established. It"s a consultative body which whective is 10 strenghen dialogue between UN and the Afghan Authorities on all issues periaining to operational arrangements of international assistance to Afghanistan. It resulted from a recommendation by lla UNDPA in April 97.

The first JTC met in Kandalar. the Kabul authority discarded the JTC idea. and those in Jalalabad expressed interest in it.

- "Mlopll issued al directive to all major hospital in Kabul requesting hal admission of female patient " ias to stop immediately and that current female staff were to cease work at the hospital. it proposed an allematice structure the Rabir Balkhi hospital. The transfer to this hospital has a risk of resulting m incriased mortality and morbidity for women in Kabul.

$N(i()$ and ICRC protest against this more segregated policy but WHO enclorsed it. it is responsible for the administration and rehabilitation of the Women hospital.

19: emergency meting between UN agencies and Task Force to discuss the maller

- 2i-i)sept workshop Strategic Framework for Afghanistan Islamabad lnchading all stakcholders

Oetober

- "oclober Adsanced medited version of Strategic Framework. not accepted

- In ATF: WHO is asked to clarify its position and to state that Taliban policy of segregated health came is mancceptable IVHO is requested to sit with the Taliban and convince them to change their policy. a laillh stantegy for Kabul in lill cooperation with NGO and ICRC will be drawn up under the ladership of IVHO.

- Revised stalcment of WHO :

- Establishment of Technical Working Groups in Easten region

November

- Early Norember: second diali of Strategic Framework for Afghanistan

- November Strategy for assistance to Afghanistan: sustaining livelihoods. discussion paper for the 7 $\mathrm{Nos}$

- 12-23 Cicnder mission lanuclied upon a recommendation of ATF and UNRCO. led by Angela King. "I amled al assessing the gender silnation in Afghanislan and at reaching an agrecment on a common set ol pagntatic guidelines. report issuc on

- $11 \mathrm{H}(\mathrm{d} / \mathrm{L}$ onors mission to assess how women's heallh is affected by llac interagency hospital rehabilitation project led by WHO. WFP and are also pat of the project.

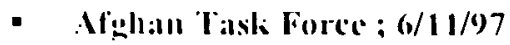

Discussion on second daft strategic Framework for intenational assistance 10 Alghinistan

Decisions hatt it does not represent a common view point and could not be acceptable

Endorsement of "Strateg! for assistance to Afghanistan: Sustaining Livelihoods" from Michacl Keallu! as a basis lor liuther discussion

- If mcetimg Alghan Task Force/ WHO mission: 
Vice minister of health gave strong commitment that the previous decision on segregated health had been revised by the sliura.

Donors stated that WHO's policy had been questioned whether they were compatible with UN principles. WHO denied having worked against the principles and UNOCHA said that one should admit laving been wrong.

- IS: heilth commission set up in Kabul by WHO : participants. WHO. ICRC. UNDP/UNOCHA. MoPH. supreme court. ministry of promotion of vice and prevention of virtue, ACF

The purpose of the commission was to reach an agreement on how to provide support for health services according to the Sharia

- Afghin Task Force 21/11/97 Strategic Review meeting

- 27 issue of the revised "draft Strategy for Afghanistan"

- Statement by Mr. Akashi on humanitarian crises and development UN General assembly: he stresses the need for a liolistic and strategic approach and that the political, lumanitarian. social and material reconstnetion lave to be addressed together

December

- 3: ASG mecting in New York,

ACBAR was invited to represent the NGO community

They emphasised the need for peacemaking and peace building efforts to be complementary. strategic Framework exercise provides the occasion to link political and humanitarian activities.

Criticism of the agencies that tolerate human right violation under the aegis of pragmatism

Endorsement of the Strategic Process (text Strategy for Assistance) but common funding mechanisms were refused. donors felt responsible for ensuring that their contribution are consistent with the strittegy and for offering consistent advice and instruction to the governing board of UN agencies

So that the strategy can be pursued purposefully

- 11 ATF decision to totally revise the Consolidated appeal so that it is in line with the Common Program approach.

- UN General Assembly resolution

it recommends that the UN agencies should coordinates their humanitarian action particularly regarding a consistent approach to human rights

it supports the secretary generil 's appeal to establish a solid international framework to deal with the external aspects of the Afghanistan question.

1998:

\section{Foluruary}

- 4 Consolidated appeal 98, second version

\section{- Meeting Resident Coordinator Officers in Herat}

to discuss the practical implication of the Common Programme and the 99 appeal for regional coordination. agrec on a draft delinition of the Common Programme as a "transparent and responsive process. defined by commitment to agreed objectives. principles and priorities. which optimises both indigenous and external resources to achieve a measurable impact on the lives of Afghans". They proposed that a draft Common Programme document be written by a group of 5 persons. They also define the role of the RCOs

\section{- Meting open forum Peshawar12/02/98}

Alfredo Witchi Cestant explained the PCP process to the NGOs

- March 1998 ACC meeting on SFA I did not found any summary

- Heallh plamning workshop 15/2-2/3

NGOs. MoPH. UNICEF. WHO 
The : am wiss to identify key health problems and decide prioritics for 98

The recommendations stated that the international community should work in full cooperation with MoPH.

March :

- Mission Report of Under secretary general DE Mello

He recommended that the Common Program and the Strategy be further developed and supported, that UN Agencies move towards common premises. he complains about the lack of senior UN staff in Kabul.

He insists on having a common approach to humanitarian aid instead of the current lack of colicsion beween those who advocite a more pragmatic approach and those who adhere rigorously to principle.

The common denominalor of UN in Afghanistan is the promotion of human rights, in this regard the capicity of the UN needs strengthening.

Humanitarian efforts need to be part of broader political efforts

- Afgham Task Force $18 / 03 / 98$ on Mahram edict;

All stakcholders agree to lake the same line

In a memorandum to USG De Mello, the Afghan Task Force states that responding to the Taliban policy must lie with agencies headquarter's and national capitals. it suggests that Ambassador Brahimi address the issue. Every one agree that it is time to draw the line with the Taliban

- 10-11/(1)3/98 why relief workshop KABOUL

it ainted at improving the aid delivery in Kabul. participants agree on common definition of problems and on common strategies

- UN headquaters Common Program team:

It included : J. Mouchet UNHCR, Zarin Naqvi, world bank, j. Renninger UNDPA, Tom Tomsen DACAAR,M. Keating, Paula newberg.

The office of the residem of the humanitarian coordinator. ask them to write a document clarifying the aim. the principles tor the stakeholders. policy underpinning the engagement of the international community. priorities and programming methodogy

They should also give institutional arrangements recommendations and time frame

- 24 UN suspends its programms in south western region, temporary evacuation of UN international staff from Kandahar

April :

- 2 o-diays working sessions of the PCP team. they meel various stakeholders

- 21 press release of UN secretary general

Humanitirian action is not a substitute for political will or military action: therefore all the arms of organisation will act in unison.

- 24 issue making a reality of principled common programming

Mạ̣ :

- 1 issue document on principles gender and capacity buildings

issue document on Monitoring and evaluation

- $\quad 05 / 98$ memorandlum UN/Islamic Emirate of Afghanistan:

NGO expressed serions concern and frustration regarding this agreement, which is croding the liberty of the UN and NGO. The Taliban have managed to gain significant advantages, the MOU allows tmansfer of residence o $n$ request of the authorities, the text is not clear and make the authorities believe they can control the employment of local staff. Donors are also unhappy with the document that they consider as a $I^{\text {st }}$ step toward Taliban recognition.

- 27 mecling international NGO/ coordinating body /Keating ON CP in Pcshawar/

NGOs expressed their support for common programming but also their concerns that the details of implementition should be clarified regarding : the composition of the APB and RCB, their role.

the regional division is not relevant (do not reflect road and communication realities nor the difference betwen Kibul and its hinterland). NGOs stressed that implementation sliould not be rushed

Lack of resource from the NGO 10 contribute as much as they should to CP 
Monitonng and evaluation must be an independant element

Some wonder whether there is room for islamic donors and whether the UN agencies are really committed 10 CP

Donors complain about the considerable disharmony anong the assistance community

- UN refuses to open Acbar oflice in UN Jalalabad coumpound for security reasons

- London ASG

Pronk pushed UN agencies and gain their commitment to the PCP approach

Donors supports the PCP process and urge the assistance community to implement it to gain greater colterence and efficiency in the aid delivery. Some of the donors agree that their financial contribution will only be miade in the context of common programming

Europe and Canada insisı on the lack of coherence of the UN system.

Link betweon political stglategies and humanitarian assistance strategies: Canada stresses the potential problems if the links are too strong. 'US field mission focused on gender issue and insist on the difficulty to distingnish lifisiving fron life sustaining programs in the field. Finland recommended avoiding an over burdening bureaucracy

Donors cimind the amomnt of money they gave to coordination. European Union stated that donors might use sanction to ensure a common programming approach. They stresses on the necessity of a medium rather than short term approach.

The coordination among donors is not improved. neither is a proposal given to ensure longer term funding.

June:

- 1-2 common programming workshop in Herat

attended by +1$)$ representatives of the UN and NGOs

A suggestion that a full time independant staff could run the RCB and that it would negate the need for $\mathrm{RCO}$ and NCB but other argued that it would not work relations with locil anthorities they should at least be informed and involved when interested in the lhemalic and lcchnical Working gromp

role of RC'B shonld be to support the coordination and thematic groups which have to be stengthen provide policy guidance, propose overall regional priority

composition should be small (7 people maxi : RCO, 4ngo, 2 UNagencies ICRC as an observer)

- $3-4 /$ common programming workshop in Jalalabad

the discussion locused on the role and composition of the RCB

agree that it shonld have a facilitating role in the planning function, form a link between TWG and APB RCB should lacilitate and not dictate

problems People did not read preparation document and. few people were presents so few was achieve Teclnical Working Group are renamed Coordinating Group to include not only sectoral groups but also crossculting thematic groups and cross cutting strategy group

- $\quad$ / common programmine workshop in Kabul

- establishment of RCB in Herat, it never worked

- $18 \mathrm{CP}$ meeting Peshawar for Central Highlands

Central Highlands ivas chosen as an accelerated test for common progranmung

the mecling was aimed at discusssing the international community short to meduim term objectives for the incrnational community in the regioncentral highlands

July: :

- 3 july Acbar gencral assembly: presentation on monitoring and evaluation and how it will fit in (common programming by Stacy Gilbert and Peter Tanıs, Undp Consultants 
- International NGOs force to leave Kabul, for not relocating to Polytechnic

- UN suspends all hut life-saving activity

- EU suspends all activity in Kabul

- 21-23/07 common programming workshop in Bamyan ESTABLISHMENT OF rcl Bamyan but it never worked dne to further evacuation of expatriate staff

- 311/07/98 consolidated appeal mill term review

Health education and Narcotics were proposed as priority sectors for common programming

\section{Anginst}

- Ifix UN Under secretary general de Mello no conditionnality should be applied on life saning programms

- linal version of de Stategic Framework submitted by Louise Frechette UN Deputy Secretary General to all heids of UN algencies : Strategic Framework for Afghanistan

- 9 summary of selected written reactions received to the document entitled making a reality of PCP

- 16 NGO cracuation

- 18 US missiles

- 19) UN evacuation

September

- 7 UN acitng coordinator suggetsted that the aid community focused on the 5 themes and objectives proposed int the last version of the Stategic Franework for the preparation of appeal 1999.

- Set up of the 5 thematic groups

- $8 / 9 / 98$ meeting ATF on the role and membership of apboard

- 10/9/98 CP workshop with NGO's in Peshawar

- $15 / 09 / 98$ meeting extended Task force on constitution APB

issues discussed the role of the Afghan authorities. of the $\mathrm{UN}$ and the $\mathrm{UN}$ resident coordinator, nature of coordination.

implemeintation of PCP decsion to focuse on particular regions and sectors

- 20 Open forum ONG Keating

- 29/09/98 Islamaball meeting extended Task force

formulialed and finalised role of A.P.B.. name its clanged from Afghan Programming Board to Body

October :

- International NGOs slowly return to Afghanistan

- 1/10/98 UN Next steps paper ;sets out the strategic and operationnal clallenges facing the UN in following months and provides the basis for engaging with the Afghan authorities in a principled manner. It reminds that the overarching goal of the UN in Afghanistan is to faciliate the transition from a state of internal conflict to a just and sustainable peace through mutually reinforcing political and assistance initiatives

- thematic workshop for the preparation of appeal 99

- 23 Security agreement hetween the Taliban authority and the UN

November :

- New UN coordinator: Eric De Mull

- 10/11/99 First meeting of A.P.B.

chaired by Japill. present 17 donors. UN Agencies :15-1 +1WB ; I ICRC :IIOM ACBAR 8 : ANCB : 5 : ICC: : :

composition : donors will agree among them the member A.P.B.. UN coordinator will discuss the criteria for membership of UN agencies. they might be less than 15. there will be 15 reprensentatives of NGOs chosill among them

stituts of A.P.B. 's decision :it makes recommendations which will be binding to the UNAgencies draft constitution is approved

- UNICEF a new representative has been appointed ( check who and when) 


\section{December}

- 7-\$/12/98 Afghin Support Group meeting in Tokyo;

7-8 ASG Tokio

15 donors a new member: Switzerland +UN agencies + NGOs

the group underlined importance of closer cooperation between political and hemanitarian activities.

The debate focused on the security issue donors said they would encourage the NGO to support the UN security position. UK and US were against the return of expatriate staff claiming that security NGO return undermined the UN efforts. EC and Norway agreed on the need for a compromis but wonder wether it is possible. UK opposed to the reurn and says that common progranuming gives the answer no individual gorermmetn view should cloud the consensus

Common progranming : donors support the efforts to prioritize proposals, and call all the players to engage in the process. The group siys it is committed to strengthen the capacity of Afghan NGOs. The challenge is now to ensure that architecture and stakeholders commitment are translated into measurable positiv impact on the ground. They stressed the importance of human rights, with a special interest for women.. They reaffirm counternarcotics as a cross-cutting principle, to be integrated into the conmon programm Donors concerns

US stresses on terrorism. it wants gender. narcotics and HR issues in the appeal

Japan fears that the contlict might spread but countionned against conditionnality in dealing with Taleb Cannada and Denmartk said that priorization must be improved. Denmark a common policy is needed for all agencies

Netherlinds and Canada asked for more Afghan involvment, EC stated that more cooperation is needed between UN and NGO

Humlin rights : participants express grave concern over human rights abuses ; US The Netherlands, Germiny and UK said more work is needed to promote human rights and gender, human rights operiationual plan should be a priority. Acbar raises the issue of wether Humanitarian aid should be conditiounal or not. how stringent conditions should be. No consensus was achieve on this issue

- $\quad 10 / 12$ lannch of Cousolidated Appeal 99,pledges US\$ 185 million, US\& 115 million sought for priority activities. the priorities were designed by $E$. De Mul on his own due to lack of consensus among players

1999

- march second meeting of APB

Junce

- Third meeting of APB

- 21-22/116 ASG in Stockholm

- meeting $s$ of UN/NGO APB members

- 99 consultancy on NGO coordination by Arne Strand

$\cdot$

UN slowly return

July allgust: regional workshop on PCP and CAP

August

constitution of RCB central region

September:

Meeting of the Thematic group in Islamahad and Peshawar to prepare appeal 2000

October

Interthematic Groups meeting

15 :deadline for projetcs submission

selection of projects through TG

November

A.P.B. meeting in Food security heallh and education

Latunch of the appeial 
Annexe 6

L'activité des coordinations

General discussions on role and achievements of coordinating bodies 


\subsubsection{Constituencies}

The coordinating bodies, as member organisations, represent different constituencies within the
NGO community: - ACBAR is the only coordinating body to represent the interests of Western
NGOs, as well as Islamic and National NGOs.

- ANCB provides a "platform" for advocating the interests of national NGOs.

- ICC represents the interests of a small but professional group of agencies joined by a common religion.

- SWABAC represents a large group of agencies based in Quetta and Kandahar.

- NCB represent agencies operating in Western Afghanistan.

Member groups show a varying degree of commitment towards their coordinating body although all coordinating bodies have a satisfactory show of attendance at organised meetings All coordinating bodies have set criteria; for membership, although no coordinating body has coordinating bodies have rather listed various stand of what constitutes an NGO. Instead, the to in order to obtain and maintain membership. Dards and obligations which are to be adhered do not fulfil these obligations, only ACBAR and SWABAckowledgement that some members criteria to justify termination of membership. have reported a reduction of members. 


\subsubsection{Mandates and Major Differences}

Turning to coordinating body mandates, as outlincd in their respective charters, all five coordinating bodies' mandates, broadly speaking, are similar and contain no opposing areas of interest. All mandates emphasise the need to maximise bencfits and to minimise duplication of assistance, and that this is to be achieved by information sharing and contact between relicf actors. However, there are three important differences which do exist between the respective coordinating bodies, outlined as follows:

\section{A Differentiation between NGOs and Contractors}

In their Charter, ACBAR has acknowledged that both NGOs and contractors may have membership. ACBAR permits both to be members on condition that the requirement of an annual budget of US\$50,000 is met.

\section{Relationship with Afghan Authorities}

Only ANCB has a clause in their Charter which explicitly states that "...the coordinating bou'y respects the laws enacted by the central government of Afghanistan." While ICC expresses a desire to have a good working relationship with the Authorities, and NCB and SWABAC have established local contact with the Taliban, none of these organisation have officially declared rispect for all laws of the Afghan Government. ACBAR has clearly stated their opposition to proposed laws and regulations, for example, in connection with the MoU issue. With respect to that particular issue, the Executive Director of ACBAR has stated that ACBAR's stance has resulted in a favourable change, for NGOs, to the contents of the MoU.

\section{lacilitated or Controlled Coordination}

ANCB's Charter contains a chapter on obligations and dutics of members. The chapter states that: "Member NGOs are to follow all the policies and standards enacted and prepared by INCB ...". This allows ANCB to operate very controlled style of coordination on its members, including policy issues. SWABAC's close cooperation with the Department of Planning in Kandahar is also another means by which a more controlled style of coordination could be insured. By contrast, ACBAR, more than any of the other coordinating bodics, most clcarly liwours the opposite approach, namely a facilitating role. ACBAR's Charter states that: "Member organisations have complete autonomy".

\section{Activity Levels}

It present, all five coordinating bodies are involved with a certain" number of activities, whisugh it appears that only ACBAR has sufficient financial resources to provide a morc iunprehensive service to their members, and fulfil the aims set in their organisational charter. llwever, as a result of disputes with the Taliban authorities, ACBAR is now restricted to "whing from Pakistan only. That being stated, it is the only coordinating body which has, in cient ycars, extensively prepared and distributed information material in English, documenting wit only its own activities, but also the achievements of the entire NGO community. This, of "Mirse gives ACBAR an advantage over the other coordinating bodies when it comes to being waluated or seeking funds and, therefore, gives the other coordinating bodies a challenge to uprove their presentation. This is one indicator of an apparent and articulated compctition : isien the coordinating bodies for recognition and funding which includes, for example, the id to spend large amounts of money to be represented in the field and attend donor meetings.

$\therefore$ "uould be impossible to miss a conspicuous lack of trust and cooperation between the .."ldinating bodies which has arisen for a number of historical, personal and professional 
rcasons. The situation has deteriorated as a result of the exclusion of certain groups from international meetings, as well as disagreements on how best to relate to the Taliban Authorities. :

\subsubsection{Services to Members}

Following on from the above, it is of interest, therefore, to review the level of services provided by coordinating bodies to their members. This seems, once again, to depend on respective financial situations. ACBAR's publications have already been mentioned. In addition, ARIC maintains a good library in Peshawar and the ARIC Box Library extension project (ABLE) provides a mechanism for the distribution of information and reading materials. None of the other coordinating bodies can offer comparable services on a continuous basis, since implementing such projects depends on specific and infrequent funding.

With the exception of NCB, all the coordinating bodies have prioritised NGO staff training, although actual implementation of that training varies. Only ANCB has established specialised training courses for female NGO staff. A current UNDP initiative, which has as its aim training for both UN agency and NGO national staff, indicates that funding will possibly be available for larger capacity building projects.

A number of practical service tasks have been mentioned, such as organisation of membership cards or visas, which are provided to all members, irrespective of whether they are groups or single agencies. There have been no recorded differences with respect to both the willingness and ability of each of the coordinating bodies to provide such services.

\subsubsection{Commercial Activities}

There are two different types of commercial activities undertaken by the coordinating bodies The first involves being "contracted" to provide services for project implementation, as has been the case with ACBAR and SWABAC. Although this brings with it additional income for the coordinating body, there is a danger first, that the coordinating body may become more of an implementing organisation than a coordinating body and second, that possible conflict could arise between a coordinating body and the respective members they are supposed to both represent and control. Involvement of a coordinating body in contracted activities requires a high level of transparency if the practise is to be acceptable to all its respective members.

The second type of commercial activities are those such as the ACBAR run printing press, HERC and Survey Unit. These three activities were taken over from member agencies on the grounds that ACBAR should a) provide a service to the member organisations and b) generate income for the coordinating body. Without going into detail on the achievements of these services, which were not evaluated, it was apparent that some members question whether the resources and time spent running those services were justified by the potential income such activitics could generate. 
Annexe 7

Making a Reality of Principle Common Programming

1. Résumé de texte

2. Structure 
Malking al reality of principled commonon porogramming

If délinil le PCP comme un «mécanisme destiné à établir les priorités de l'assistance internationale ainsi que des programunıes et projets basé surr des buts. et principes communis et sur les besoins exprimés des Afghans "

\section{Levedements de base}

- Toutes les activites et projets d'aide devront être clairement reliés à cles -specific programs>

- Les prorites seront délinies en fonction d'analyses actualisées de la situation el des besoins clairement cypromes des bencliciaires

- Les programmes feront preure de leur adhésion aux principes et anx nomes opérationnelles communes

- les priorites seront définies aux nivcenux régional el nattional en fonction de Cf.ci dessus

\section{Lisprimcipes}

- respect der droits de l'homme de la chane des NU. de UNDUDH. Convention sur Elimination de toutes formes de discriminations à l'encontre des femmes.

- laride doil saltaquer aux discriminations el sassurer gu cllo ne les crie pas ni ne les perpélue

- someraincti de lectat alghtan

- Les panticipants aux programmes doivent être prolégés de l usige arbiltaure de la force

- Tot:ile impartialité

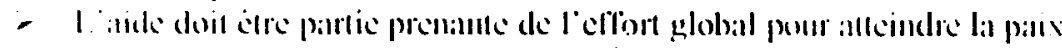

\section{3. () gyilnisiltion instimutionnclle}

L. Levie prevoit une organisation institutionnelle complexe que je ne retranscris pas ici dans la mesure où L a strucurc presicic a dans les grandes lignes élé mise en pratiçue el où le processus étant évolutif tout n'est pas encore delinitivement fixé. Cr. Organigramme prévu.

Ainsi le role. la colnposition. les fonctions des différents organtes el leur relation entre eux ne sont à ce jour pas wajours ctarement appartents. 


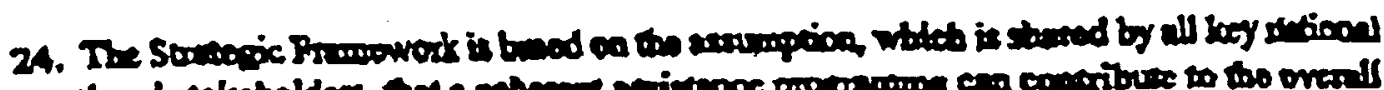

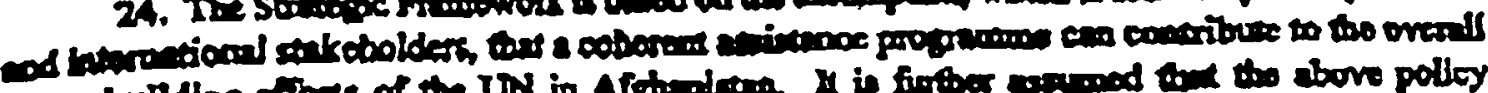

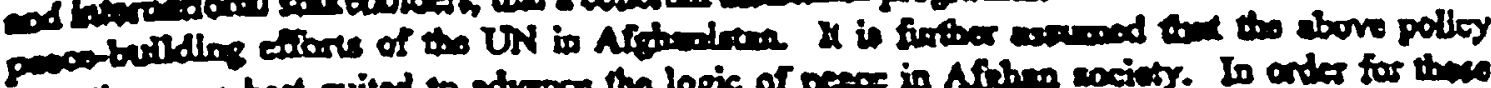

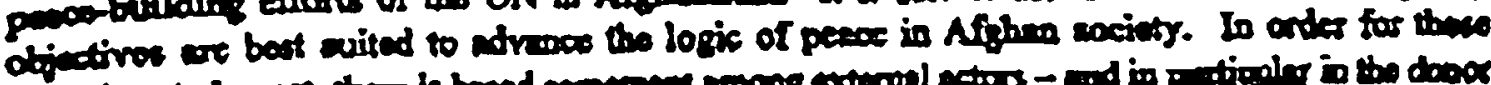

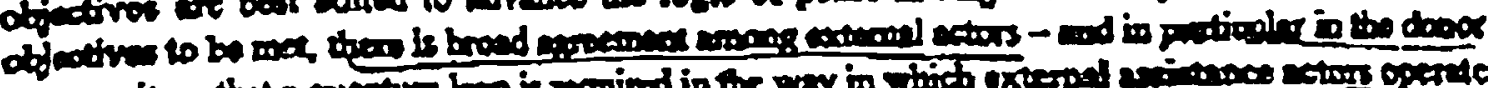

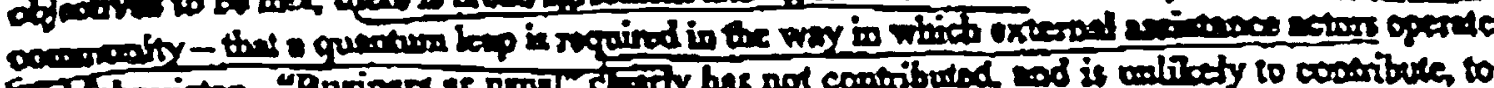

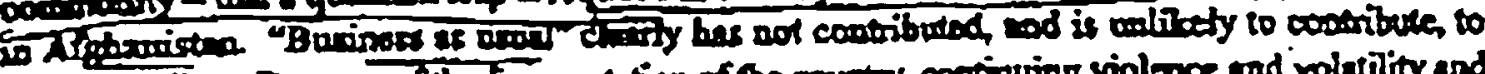

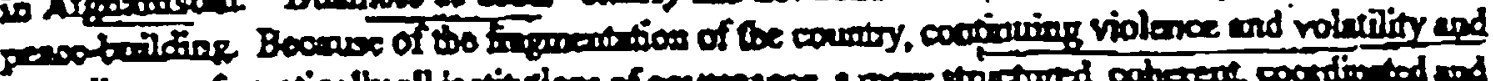

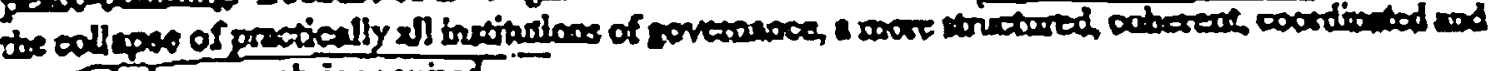
priokiplod wpronob is sequired.

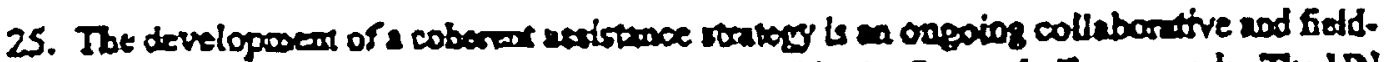

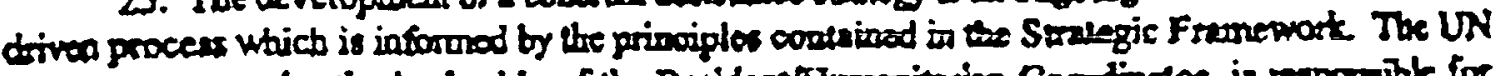

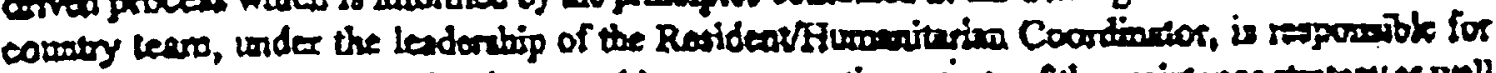

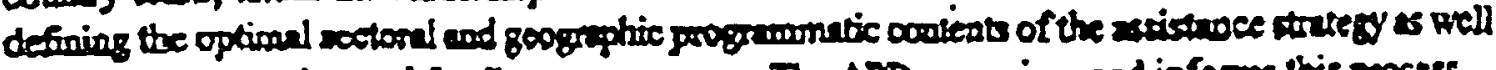

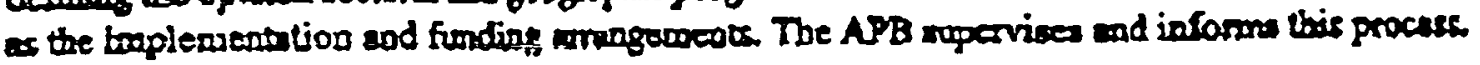

26. To be effective, nuch a coracon feld-besod progranoing process musp ensure:

- the prioritization of scuroe oxterral resauroes so that priority sectored end geographical roods, as woll an those of divadvantagod groups, can be coet is a cobered cononer,

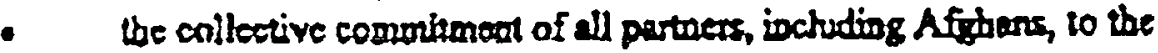

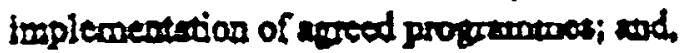

- The agroment of all permers oa the role of ibe UN Coordinntor in manging this propess on berhulf of the collecivity of exterasl wecoss.

27. In the Spring of 1998, efforts aimed al weting the enge for a wore coberwat and

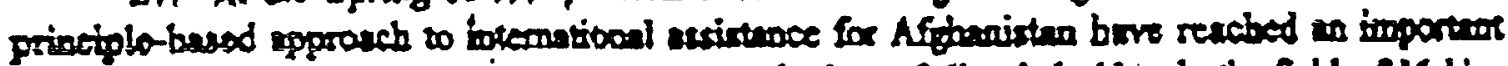

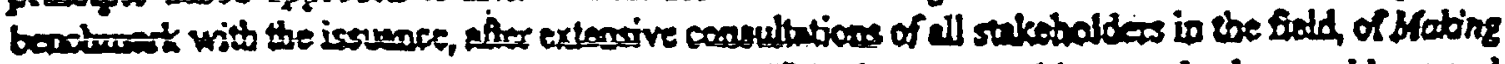

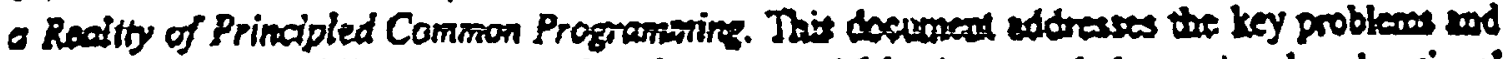

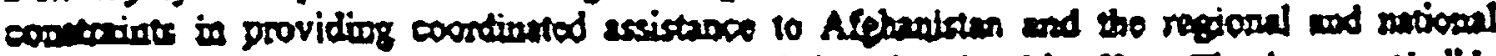

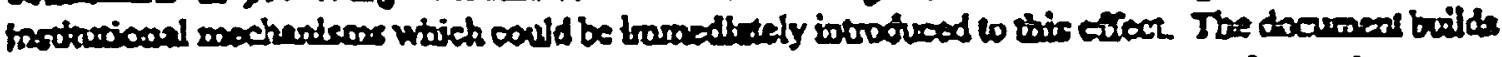

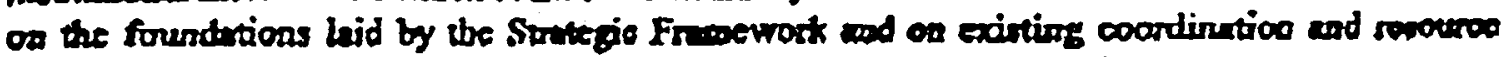

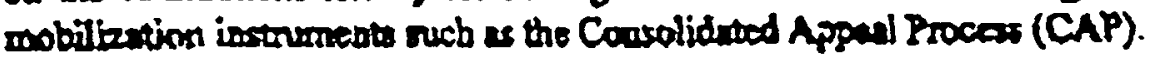

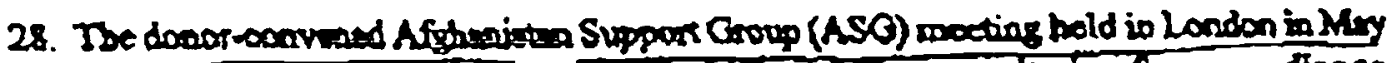

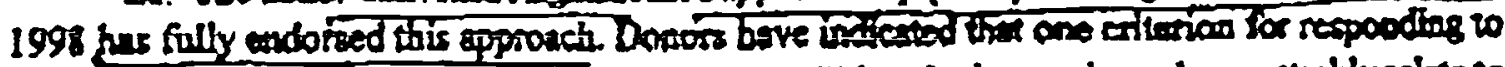

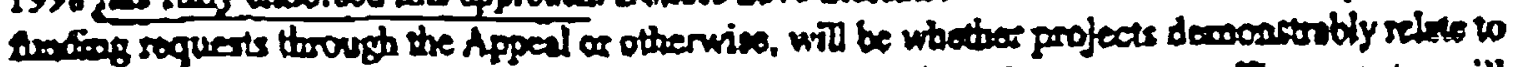

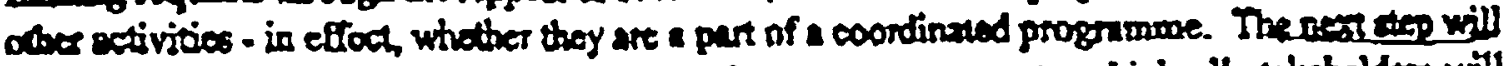

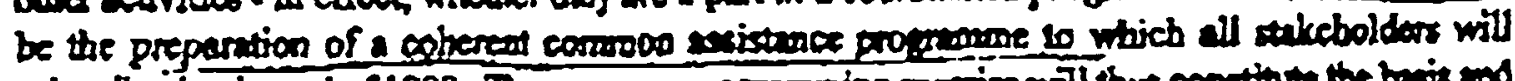

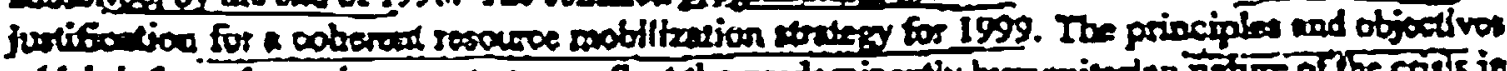

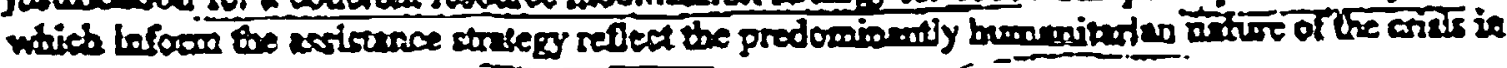




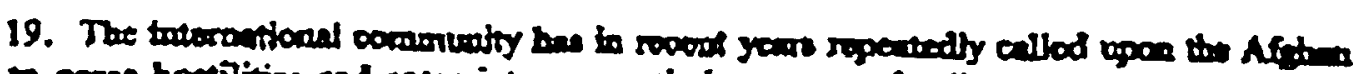

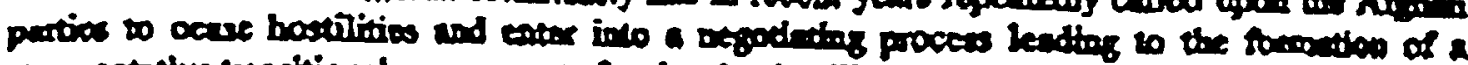

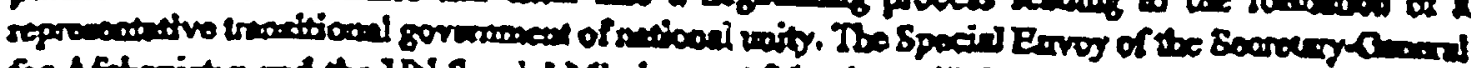

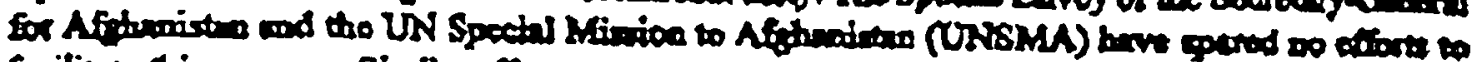

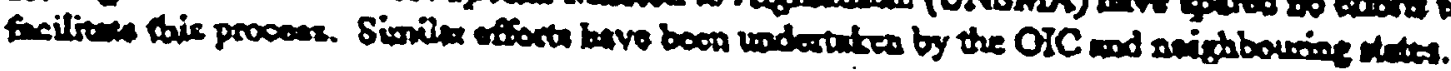

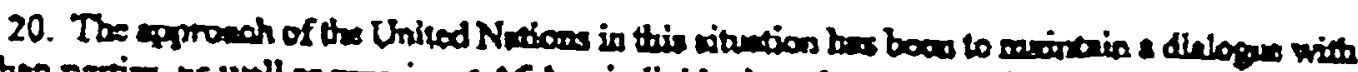

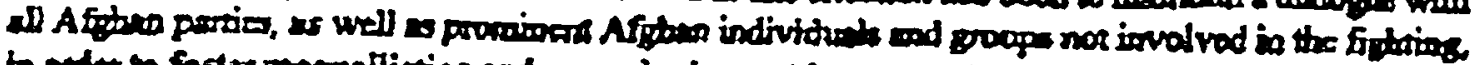

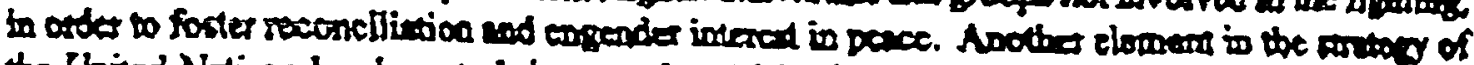

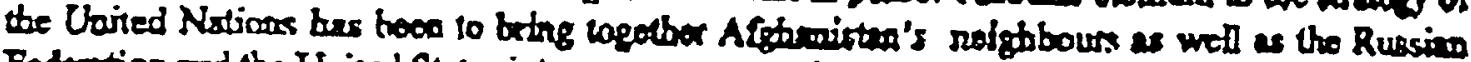

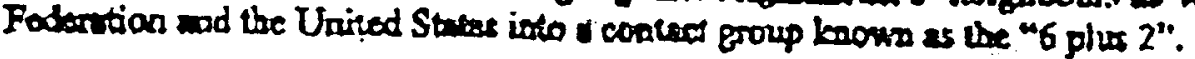

\section{The Unitod Nations politeal stming bus the following objoctives.}

- to achieve a cecention of bostitities:

- to seck a regional political consensus in suppon of the peace process;

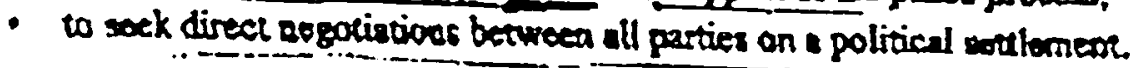

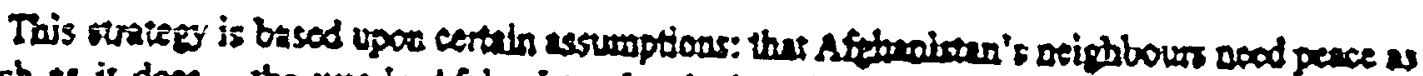

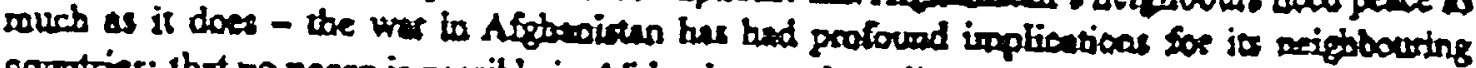

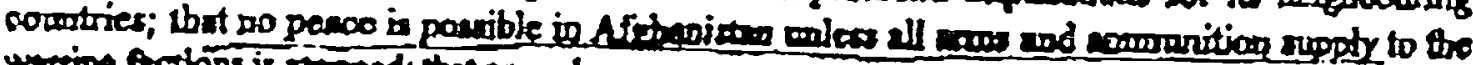

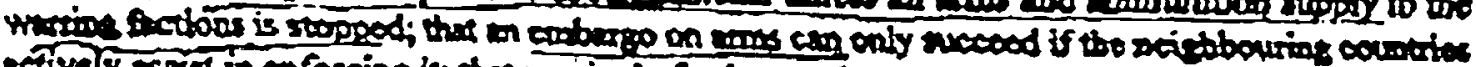

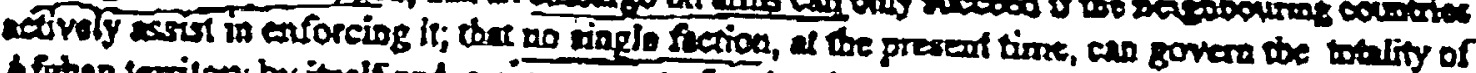

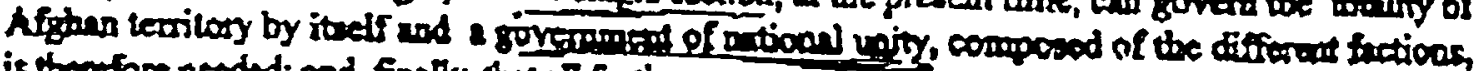

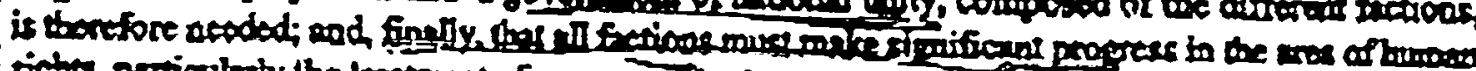

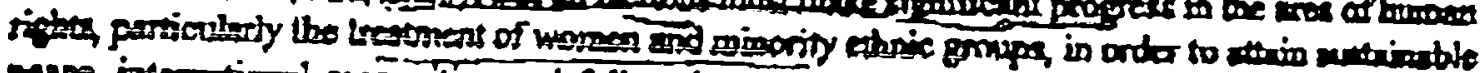

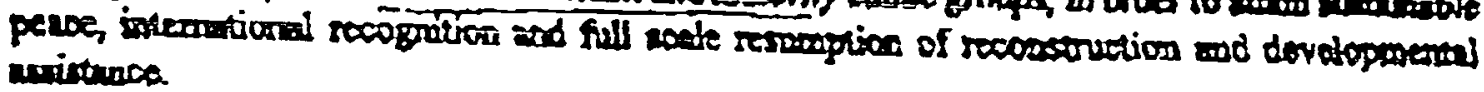

\section{Aspistance Strategy}

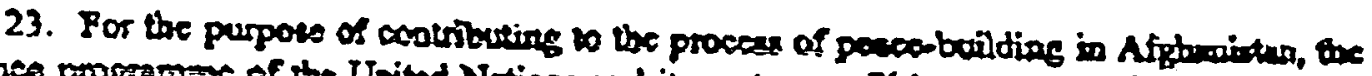

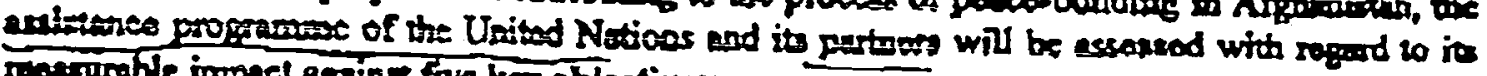

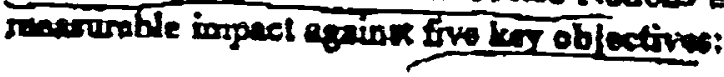

\footnotetext{
- the alleviation of branan strefiecing

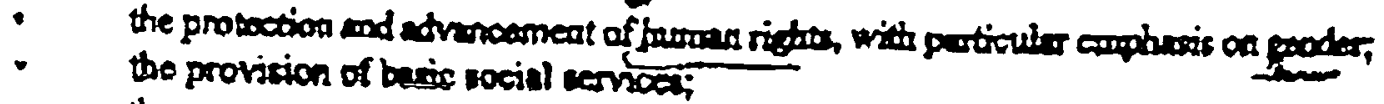

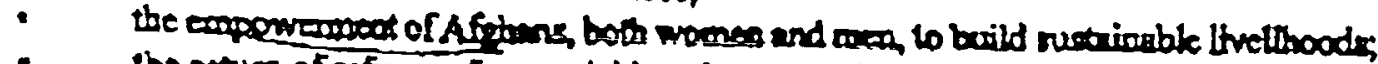

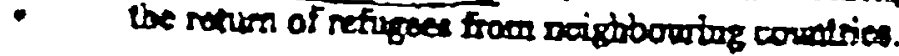


Annexe 9

Term of Reference of Thematic Group : Return of Refugees 


\title{
TERMS OF REFERENCE: TG on RETURN of REFUGEES
}

\author{
Composition: NGOs, Agencies, donors Chair: UNHCR \\ NGO Ombudsman (to be selected by NGOs)
}

Consultative Group: Nominative. Chair: monthly rotation

\section{Secretariat: UNHCR}

Meetings: TG: Quarterly (also with invitation to non-member donors)

Core group meets monthly or upon request of a member (others can be invited as required by a specific issue)

\section{Objectives:}

TG:

- set overall strategy on yearly basis.

- review strategy on quarterly basis.

- prepare quarterly reports (programme achivevements, constraints etc..) for other TGs and donors. To be drafed by Secretariat.

- serve as forum for UNHCR to update on regional situation, policy changes etc...

- report to and advise APB

- act as fundraising lobby

\section{Consultative Group:}

- define programming standards and criteria within overall TG strategy.

- decide on baseline information and data needs and gathering mechanisms.

- define and implement working relationship with other TGs

- define and implement working relationship with RCBs

- serve as forum for UNHCR to update on regional situation, policy changes etc...

- prepare brief monthly report for TG. To be drafted on rotational basis.

- receives from NGOs/agencies on project constraints/problems and assists in ' finding solutions 
Annexe 10

Exemple de stratégie de groupe thématique 


\section{Empowerment of Afghans (Both Women and Men) to Build Sustainable Livelihoods, Including Non-narcotic Alternatives}

\section{PRIORITIES FOR THE YEAR 2000}

\section{Improved Food Security}

The focus will be on the following:

a) Improved land utilization:

Access to improved agricultural inputs and technology will be essential. Through increasing the capacity to locally produce improved inputs in different parts of the country, the accessibility and affordability of essential inputs will increase, especially for poorer farmers in more remote areas. This will need to be accompanied with relevant training and extension advice.

Increased self-sufficiency by supporting community based irrigation improvement projects. These projects should also provide advice and practical demonstrations on the economical and effective use of irrigation water.

b) Improved livestock production:

Livestock is an important livelihood asset in Afghanistan and represents a safe form of saving as it can be easily converted to cash when needed.

Access to quality, affordable animal health services will continue to be important. The focus will be on technical support to existing veterinary field units to help them improve the quality and delivery of services on a full cost-recovery basis.

Support will also focus on increasing fodder production per unit area through introduction of tested improved seed varieties and new fodder crop demonstrations.

c) Improved basic infrastructure:

Lack of particular types of infrastructure is a core dimension of poverty in Afghanistan. The poor condition of essential transport infrastructure constrains people's productive capacity and contributes to food insecurity in several ways: essential inputs cannot be distributed effectively and agricultural yields remain low; it is difficult and expensive to transport food from surplus producing areas to parts of the country, usually remote, that face chronic or seasonal food deficit.

The focus will be on support for the rehabilitation of key road infrastructure that supports the marketing of essential inputs and agricultural products. This type of work also generates employment and income opportunities for people with no access 
to productive assets or employment opportunities, provides improved access to social services and increases the capacity of assistance agencies to work in remote areas.

\section{Priority areas}

The focus in 2000 will be on areas facing food shortages (e.g. central, north-eastern areas)

\section{Increased licit incomes and employment (on farm, off-farm, non-farm)}

Emphasis will be placed on building on existing assets, cultural values and resourcefulness in order to expand people's choices and capabilities and their potential to make choices. The focus will be on the following:

a) Micro-finance schemes for the poorest, vulnerable households:

For this type of schemes to be effective, integrated approaches that combine credit with non-financial services - such as fund management and skills training, technical advice as well as support in social sectors - should be pursued.

b) Micro-finance schemes for poor farming households:

Credit will be provided in cash or in kind (inputs).

c) Capacity building for management of micro-financing, micro-entrerpreneurship, micro-enterprise.

\section{Priority areas/groups}

- The poorest/most vulnerable households, both in rural and urban areas:

- widows, female headed households, disabled

- asset poor landless and sharecroppers

- itinerant harvesters/labour

- unemployed (with or without skills)

- Poor farmers - with underutilized or illicitly utilized assets

- Build on existing programmes/projects and gradually develop programmes for resource poor, under-serviced areas.

\section{Good governance}

The emphasis will be on strengthening community organizations, taking into account existing strengths of Afghan society, in order to achieve a more supportive and cohesive social environment. Strong, inclusive community groups and networks of groups can be effective in improving the management of natural resources and the maintenance of 
community infrastructure, manage micro-finance/saving schemes, facilitate innovation and sharing of knowledge, and can ultimately influence policies at different levels.

Strong, cohesive community groups can also provide a safety net for their poor and vulnerable members through helping them cope with shocks and ensure survival and dignity during periods of crisis and extreme insecurity.

The focus of support will be on:

a) Capacity building:

- Community organizations will need support to improve their capacities to address their own needs. Depending on particular needs, the types of support would include training in participatory appraisal and analysis, management and record keeping.

- Support will also be needed to build the skills and capacity of the field staff of assistance agencies in facilitating community group formation and structure, facilitating participatory decision making processes, gender awareness, rights awareness, the importance of social sectors in community development etc.

\section{Priority areas/groups:}

Building good governance through empowerment of community organizations is a necessary component of any programmes/projects aiming to achieve sustainable improvements in people's livelihoods. As such, it can be only pursued in conjunction with support in other areas (e.g. management of natural and physical resources, micro-finance).

The focus therefore will be on rural and urban areas and communities where aid organizations are already active (and where they plan to start working).

Community organizations and networks should cover several levels: village/district in rural areas, neighborhood/district in urban.

\section{Common Programming to reduce poppy cultivation}

In order to reduce illicit crop cultivation through common programming, international agencies will continue to collaborate in 2000 to build a joint programme in selected provinces, which will establish strong local links with national programmes and policies. Through established coordinating bodies, interested aid actors will develop a coordinated, targeted and phased set of development interventions aimed at reducing opium poppy cultivation and creating sustainable livelihood strategies in Helmand, the major poppy producing province in Afghanistan.

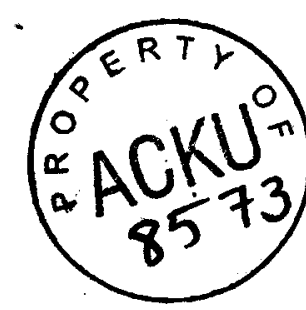


Note:

The original material azu_acku_pamphlet_hv555_a3_b68_1999: page 52 to 92: Missed The original material azu_acku_pamphlet_hv555_a3_b68_1999: page 115: Faded 